\title{
MEASUREMENTS ON INSULATING MATERIALS AT CRYOGENIC TEMPERATURES.
}

\author{
ANNUAL REPORT
}

PART I

WILLIAM E. ANDERSON

RICHARD S. DAVIS

PART II

F.I. MOPSIK AND S.J. KRYDER

F. KHOURY.J.P. COLSON AND L.H. BOLZ

\section{UNITED STATES DEPARTMENT OF COMMERCE NATIONAL BUREAU OF STANDARDS WASHINGTON, D. C. 20234}

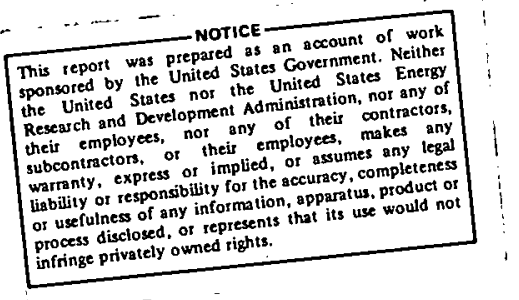

\footnotetext{
DATE PUBLISHED - SEPTEMBER 1976

\section{PREPARED FOR THE ENERGY RESEARCH AND DEVELOPMENT ADMINISTRATION OFFICE OF CONSERVATION UNDER CONTRACT NO. E(49-18)-2062} DIVISION OF. ELECTRIC ENERGY SYSTEMS
} 


\section{DISCLAIMER}

This report was prepared as an account of work sponsored by an agency of the United States Government. Neither the United States Government nor any agency Thereof, nor any of their employees, makes any warranty, express or implied, or assumes any legal liability or responsibility for the accuracy, completeness, or usefulness of any information, apparatus, product, or process disclosed, or represents that its use would not infringe privately owned rights. Reference herein to any specific commercial product, process, or service by trade name, trademark, manufacturer, or otherwise does not necessarily constitute or imply its endorsement, recommendation, or favoring by the United States Government or any agency thereof. The views and opinions of authors expressed herein do not necessarily state or reflect those of the United States Government or any agency thereof. 


\section{DISCLAIMER}

Portions of this document may be illegible in electronic image products. Images are produced from the best available original document. 
PART I

DISSIPATION FACTOR MEASUREMENTS MADE IN THE

ELECTRICITY DIVISION ON INSULATION AT

HIGH VOLTAGE AND CRYOGENIC TEMPERATURE

Interim Report for the period

Nov. 1974-Sept. 1976

by

William E. Anderson

High Voltage Measurements Section

and

Richard S. Davis

High Voltage Measurements Section 
TABLE OF CONTENTS

ABSTRACT . . . . . . . . . . . . . . . . . . . . 1 SUMMARY. . . . . . . . . . . . . . . . . . . . . . 2 INTRODUCTION . . . . . . . . . . . . . . . . . . . 6 EXPERIMENTAL . . . . . . . . . . . . . . . . . . . . . . 7 Sample Holder . . . . . . . . . . . . . . . . 8 Cryostat. . . . . . . . . . . . . . . . . 11

Standard Capacitor. . . . . . . . . . . . . . 13

Current Comparator Bridge . . . . . . . . . . . . 17 RESULTS. . . . . . . . . . . . . . . . . . . . 24 WORK IN PROGRESS: CORONA DETECTION SYSTEM . . . . . . . . . . 30 Electronics. . . . . . . . . . . . . . . 32 Data Analysis. . . . . . . . . . . . . . . 34 Progress . . . . . . . . . . . . . . . . 36 CONCLUSTON. . . . . . . . . . . . . . . . . . . 36 REFERENCES. . . . . . . . . . . . . . . . . . . . 38 RIRLIOGRAPHY. . . . . . . . . . . . . . . . . . 39 


\section{$\underline{\text { ABSTRACT }}$}

This report details progress made to date on developing instrumentation and measurement methodology for studying high-voltage dielectric losses at cryogenic temperatures. The work described has been done in support of ERDA-funded ac superconducting transmission line projects at Brookhaven National Laboratory (BNL) and the Linde Division of the Union Carbide Corporation (UCC-Linde). Dissipation factor measurements have been made at a temperature of $4.2 \mathrm{~K}$ and at stresses up to $40 \mathrm{kV} / \mathrm{mm}$. Care has been taken to insure that errors in dissipation factor measurements are less than $\pm 1 \times 10^{-6}$. Sample dielectrics have included polymer tapes of interest to BNL and epoxy spacer material of interest to UCC-Linde. When dissipation factor measurements are made at high voltage, losses at sample interfaces become important. Flexible superconducting cables are designed to have many layers of coaxially wound plastic tape serving as the insulation. The spaces between tape layers will be impregnated with helium at pressures up to $1.5 \mathrm{MPa}$. Plans to investigate highvoltage dielectric losses under these conditions are discussed including a technique for measuring partial discharges using pulse-height analysis. 
SUMMARY

The contents of the following report are here summarized for the convenience of the reader. The report covers the period from November 1974 through June 1976.

In November of 1974 the High Voltage Measurements Section in the Electricity Division of the Institute for Basic Standards began a project sponsored by the Energy Research and Development Administration to study ac losses in dielectrics at cryogenic temperatures. The ultimate goal of the project is to provide dissipation factor and corona data under the proposed operating conditions for Brookhaven National Laboratory's (BNL's) ac superconducting power transmission line (AC SPTL). Because of BNL's urgent need for engineering data and the extra time involved in designing and constructing a supercritical cryostat, initial efforts have been toward providing $60-\mathrm{Hz}$ dissipation factor data of polymer films at $4.2 \mathrm{~K}$ and atmospheric pressure. These measurements, although only a first step, do provide valuable data at the electrical stresses proposed for the AC SPTL. Preliminary dissipation factor measurements on layers of polymer films suggest that losses at interfaces may dominate the intrinsic dielectric losses of the polymer films. These interfacial losses are probably due to partial discharges. We have begun the design and development of a system to study these partial discharges using the pulse height analysis technique.

Besides the measurements on the polymer tape insulation for the BNL SPTL, we have provided Union Carbide Corporation, Linde Division (UCC-Linde) with dissipation factor values for epoxies used as spacers in their helium-insulated cable.

In order to accomplish the necessary measurements, a cryogenic facility was built in the High Voltage Measurements Section. This consisted of a conventional glass double-dewar system. High voltage was introduced into the cryostat by means of a vacuum-insulated bushing which was discharge free to $8.5 \mathrm{kV}$. All experiments were performed with the sample material immersed in liquid helium 
at atmospheric pressure. Size limitations of the dewar necessitated the use of

a small (10 $\left.\mathrm{cm}^{2}\right)$ parallel-plate capacitor as sample-holder.

Electrical measurement of dissipation factor was made by comparing the loss of the sample dielectric with that in a commercial compressed-gas high-voltage capacitor. This comparison was made with sufficient accuracy by using the NBS current comparator bridge. Samples formed the dielectric of a three-terminal stainless-steel capacitor whose design incorporated ASTM recommendations for dissipation factor measurements. Due to the small dissipation factor allowable in superconducting cable insulation, an accuracy of $\pm 1 \times 10^{-6}$ was desirable in our measurements. Success in this was achieved through a meticulous absolute determination of the dissipation factor of our compressed-gas high voltage capacitor.

As an initial consistency check we measured the dissipation factor of liquid helium at $4.2 \mathrm{~K}$. At a stress of $1.5 \mathrm{kV} / \mathrm{mm}$ the $60 \mathrm{~Hz}$ dissipation factor was $(0.3 \pm 0.7) \times 10^{-6}$. This is consistent with the fact that liquid helium has no intrinsic loss mechanisms at power frequencies.

No attempt was made to measure a representative sample of polymer tapes. Only those tapes that proved to be among the most promising from mechanical tests, low-voltage electrical tests, or economic considerations were sent' to us by BNL. Each sample was measured at least twice to test repéatability and prevent erroneous values caused by the occasional cracking of some materials.

General comments can be made about the results of these measurements. The polyethylenes and polypropylenes both have dissipation factors considerably less than $30 \times 10^{-6}$ (upper limit for BNL SPTL) at design stresses of the order of $20 \mathrm{kV} / \mathrm{mm}$. These films along with the polyamide sample, however, have poor mechanical properties at liquid helium temperatures. The polycarbonate and polysulfone films are much better mechanically but have dissipation factors in the 
range of 50 to $100 \times 10^{-6}$. An attempt by the manufacturer to improve the dissipation factor of one of these materials (green polysulfone) by reducing the concentration of sodium impurities was somewhat successful.

While the dissipation factor of most of the materials increases slightly with increasing voltage, both of the polyether-sulfone samples measured have a negative voltage coefficient. We do not know whether the cause of this is related to the well-understood behavior of oil-impregnated paper-insulated capacitors.

The dissipation factor of the epoxies submitted by UCC-Linde were an order of magnitude larger than the polymer tapes but below the $500 \times 10^{-6}$ limit for the UCC-Linde SPTL. Sample preparation (including catalyst concentration) may account for the varying results on identical materials of differing thicknesses. The apparent increase of the dissipation factor with sample thickness, however, may be a real effect resulting from the increased probability of voids or other defects. We found it impossible to make these measurements without coating the electrodesample interfaces with paraffin oil. Presumably this procedure suppresses corona at the interfaces. It has been our experience that when samples have not been coated with paraffin oil prior to insertion into the sample capacitor, the measured dissipation factor exhibits a strong voltage coefficient. Presumably this, in part, is caused by the presence of helium bubbles. The corona might be suppressed in supercritical helium. This point will be elucidated by our measurements in the coming year.

To examine further the effects of paraffin oil on various interfaces, we studied several stacking arrangements of polypropylene films. We measured the dissipation factor of three-sheet stacks of this material with and without paraffin oil on the various interfaces. As the number of uncoated interfaces increases, the positive voltage coefficient of the dissipation factor increases. This effect may be suppressed under the supercritical conditions of BNL's proposed SPTL. It is therefore our intention to continue our measurements in an apparatus which will operate at these conditions. 
The results reported to date are the outcome of measurements to determine dissipation factors in single-layer samples at high electrical stress. The actual design of BNL's SPTI calls for wrapped polymer tape insulation impregnated with flư⿱亠乂 helium. In such a system, the occurrence of corona between tape layers or butt gaps may dominate other loss mechanisms. Corona could also prove to be the most important cause of insulating aging in a regime in which "normal" thermal aging is suppressed. We have, therefore, designed and begun construction of a corona measurement system:

In order to glean more information that can normally be obtained from a conventional corona detector, our system will use a technique recently borrowed from the nuclear physicists--pulse height analysis. With this method the corona spikes are sorted by peak amplitude. The result is a.histogram of the number: of spikes of a particular amplitude versus amplitude. The evolution of the profile of this histogram as a function of time will yield information concerning changes in the dielectric. A particular profile might, for example, indicate a large void or impurity exists in the dielectric. Parameters such as applied voltage, temperature, frequency, and pressure can be varied in order to study their effect on this profile. The final profile for each experimental run will be stored on a floppy disc for future comparisons. The minicomputer used to sort the data will also be available for data manipulation such as finding the total energy content of the corona -- the integral of the histogram. The individual circuits have been breadboarded and tested but the total system has not yet been assembled.

Future work will involve extending our dissipation factor measurements to conditions proposed for the AC SPTL: Measurements will be made on coaxial samples. at temperatures from 7 to $9 \mathrm{~K}$ and pressures from 1 to $1.5 \mathrm{MPa}$ ( 10 to 15 atmospheres). The corona measurement system will be completed which will enable simultaneous corona and dissipation factor measurements. Corona inception level measurements on single layer and multi-layer polymer films will also be initiated. 
INTRODUCTION

In November of 1974 the High Voltage Measurements Section in the Electricity Division of the Institue for Basic Standards began a project sponsored by the Energy Research and Development Administration to study ac losses in dielectrics at cryogenic temperatures. The ultimate goal of the project was to provide dissipation factor and corona data under operating conditions proposed for an ac superconducting power transmission line (AC SPTL) at Brookhaven National Laboratory (BNL). In order to provide useful data as soon as possible, initial efforts have been toward measurement of the dissipation factor of polymer films at $4.2 \mathrm{~K}$ and atmospheric pressure. The engineering questions to which we sought apswers are: which polymer insulating tapes have the lowest intrinsic ac losses at cryogenic temperatures; and are these losses a function of electric field amplitude, particularly up to design stresses proposed for the AC SPTL? Later in the project, we also began measuring ac losses of epoxies. The epoxy measurements are useful to the AC SPTL program at the Linde Division of Union Carbide Corporation (UCC-Linde). The projects at BNL and UCC-Iinde are both funded primarily by ERDA.

The respective interest in polymer tapes by BNL and epoxies by UCC-Linde reflects their different cable designs. BNL's cable will be flexible. The insulation contemplated is analogous to conventional oll-impregnated paper cables in that helium plays the role of oil and a suitable polymer tape the role of paper. The UCC-Linde cable is a rigid or pipe-type cable which uses helium as the electrical insulation and epoxy spacers to position the conductor in the helium.

As our work progressed, it became increasingly clear that interfacial losses, either between layers of insulation or at electrode surfaces, can exceed the intrinsic dielectric losses of polymer tapes. The interfacial losses are probably due to partial discharge. Therefore we have undertaken the design of a corona measuring system which will, through the use of pulse height analysis, provide more information than can be gleaned by using commercially available devices. 
This report covers the period from November 1974 through June 1976. It, is divided into three major sections: Experimental, Results, and Work in Progress. The first section establishes the theoretical basis and describes our equipment for making accurate dissipation factor measurements. Also included as an appendix is a bibliography of useful references we have accumulated since undertaking the work reported here.

Other work not covered in this report included the presentation of papers at the 1975 Conference on Electrical Insulation and Dielectric Phenomena held in Gaithersburg, Maryland and at the 1976 IEEE International Symposium on Electric Insulation held in Montreal, Canada. Also the equipment used by BNL to measure dissipation factor was calibrated in situ by NBS personnel.

The principal investigators for the work reported below were William E. Anderson and Richard S. Davis, both physicists in the High Voltage Measurements Section of the NBS Electricity Division. Administrative and scientific support were provided. by Oskars Petersons, Chief of the High Voltage Measurements Section and Barry N. Taylor, Chief of the Electricity Division. Eric D. Sims, a technician, also worked part-time on this project. The ERDA program manager is Alvin S. Clorfeine.

\section{EXPERIMENTAL}

The power, $\mathrm{P}$, dissipated in a transmission cable due to dielectric heating follows the relation

$$
P \propto f V^{2} \varepsilon^{\prime} \tan \delta
$$

where $f$ is the power frequency and $\dot{V}$ the operating cable voltage. In a superconducting transmission cable where refrigeration efficiency is low, all sources of heat generation must be kept as small as possible. The reduction of $P$ to an acceptable value can only be accomplished by minimizing the product of $\varepsilon^{\prime} \tan \delta$ where $\varepsilon^{\prime}$ is the real part of the dielectric constant $\varepsilon=\varepsilon^{\prime}+j \varepsilon^{\prime \prime}$ and $\tan \delta=\varepsilon^{\prime \prime} / \varepsilon^{\prime}$, the dissipation factor. Since $\epsilon^{\prime}$ is about 2 to 3 for all polymers being considered for the dielectric in a superconducting transmission cable, tan $\delta$ is restricted to 
a value of less than $30 \times 10^{-6} \cdot 1,2$ This number is a rough figure of merit based on the economics of cable refrigeration.

Measurements of the dissipation factor of various polymer films under high electrical stress and at cryogenic temperatures are necessary in the search for a suitable dielectric. Since the design stress for the superconducting transmission cable $^{3}$ is about $10 \mathrm{MV} / \mathrm{m}$, measurements at voltages in the range of $2 \mathrm{kV}$ to $5 \mathrm{kV}$ for samples of the order of $100 \mu \mathrm{m}$ thick are needed. A total measurement uncertainty of about $\pm 1 \times 10^{-6}$ is required for meaningful comparisons of the materials.

The sample to be measured is placed between the electrodes of a parallel-plate capacitor. The capacitor is inserted into a cryostat which is filled with liquid helium. The current in this capacitor is then balanced against the current in a compressed-gas standard capacitor using a current comparator bridge. This measurement yields both $\varepsilon^{\prime}$ and the dissipation factor. Each component of this measurement system will be described in detail.

\section{Sample Holder}

The sample holder consists of the two plates forming the parallel-plate capacitor, the dielectric of which is the material to be measured. The stainless-steel electrodes are shown in Fig. 1. The bottom plate consists of an active (inner) electrode and a guard ring. These two electrodes were epoxied together then lapped and polished. The area of the active electrode is $1.05 \times 10^{-3} \mathrm{~m}^{2}$ resulting in the following relation between capacitance $\mathrm{C}$ and thickness $\mathrm{d}$ (in $\mu \mathrm{m}$ ) of the dielectric:

$$
C \simeq 9.29 \times 10^{3}\left(\varepsilon^{\prime} / \mathrm{d}\right) \text { picofarads. }
$$

Typical thicknesses of the sample dielectric range from $25 \mu \mathrm{m}$ to $125 \mu \mathrm{m}$ so that the capacitances to be measured are in the range of about 100 to $1000 \mathrm{pF}$ (remembering that $\varepsilon^{\prime} \sim 2$ or 3 ).

The capacitor is held together with two polytetrafluoroethylene plates using nylon screws and nuts.with phosphor-bronze springs as shown in Fig. 2. During the 
TOP ELECTRODE

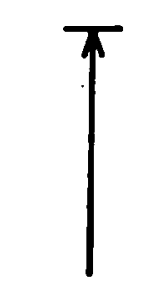

$5.6 \mathrm{~cm}$

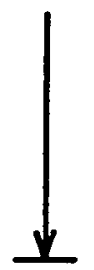

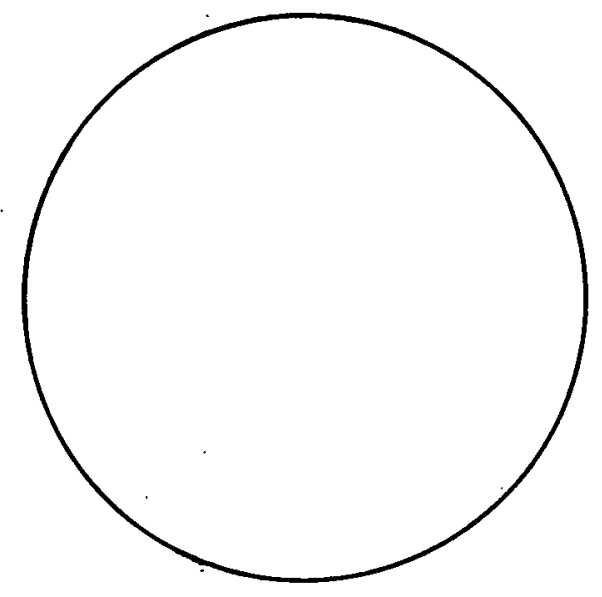

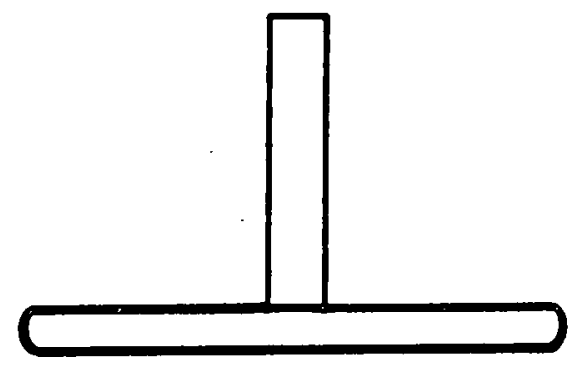

BOTTOM ELECTRODES
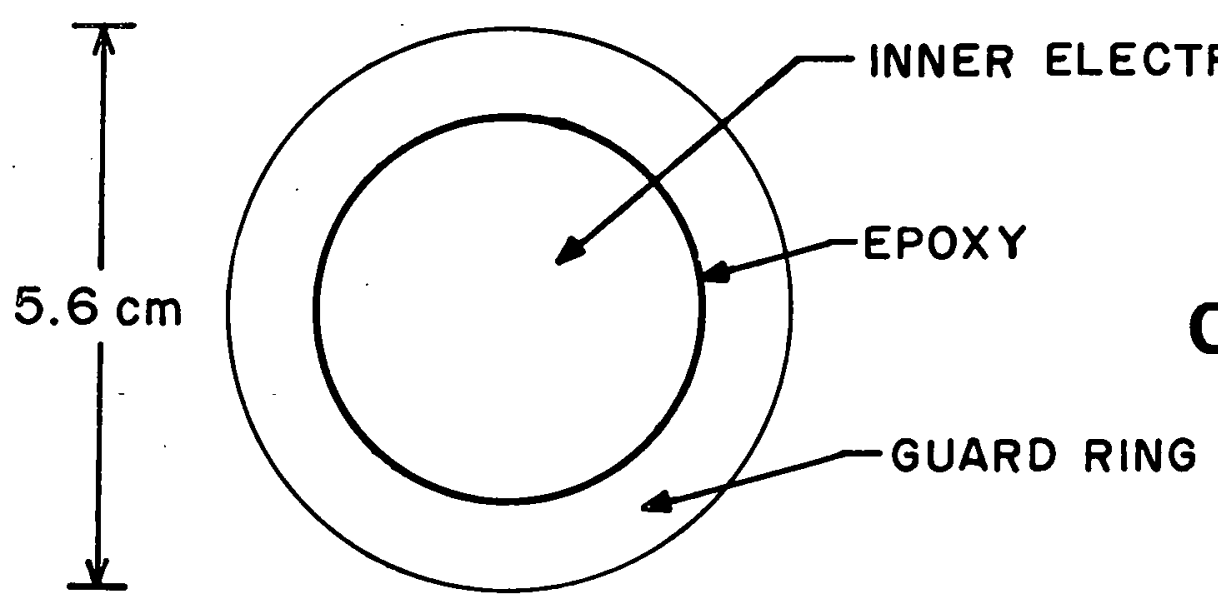

$10-3.7 \mathrm{~cm} \rightarrow$

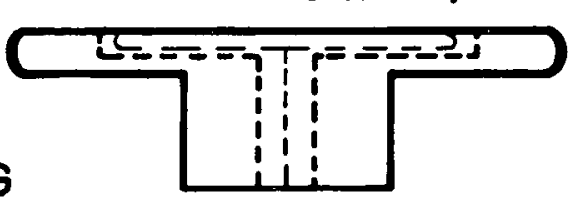

FIG I. ELECTRODES 


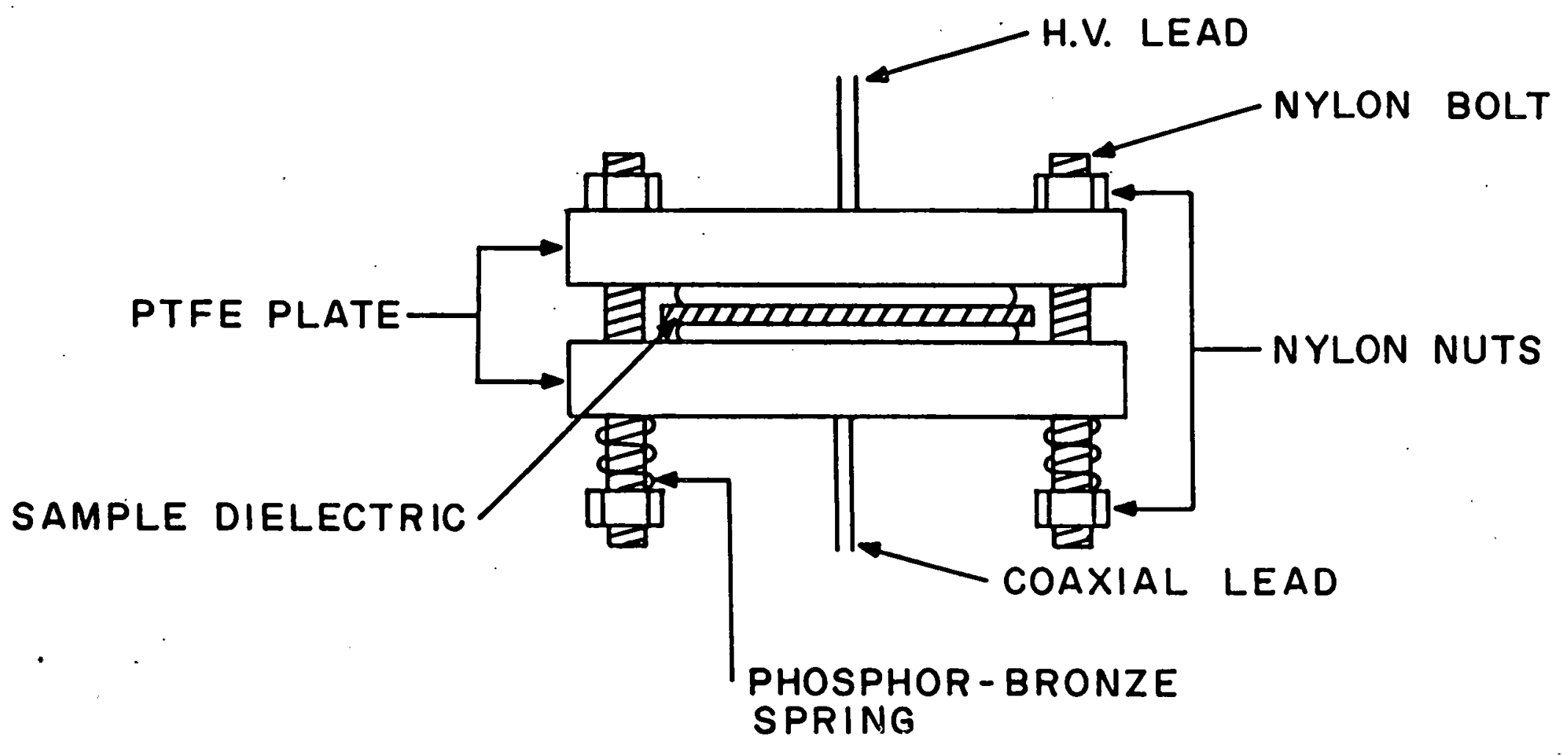

FIG. 2 SAMPLE HOLDER ASSEMBLY 
actual measurement the top electrode is energized and the resulting current from the inner active electrode is sent to the current comparator bridge via a coaxial cable. The outer shield of this cable is connected to the guard ring on one end and to ground at the bridge.

Cryostat

The cryostat is shown in Fig. 3. The I.D. of the inner glass dewar is $11 \mathrm{~cm}$ with a usable height of $1 \mathrm{~m}$. A high-vacuum feedthrough $\left(\sim 10^{-5} \mathrm{~Pa}\right)$ terminated at both ends by commercial ceramic bushings (rated at $8.5 \mathrm{kV} \mathrm{rms}$ ) is used to get the high voltage below the liquid helium level. This is necessary because gaseous helium has poor breakdown characteristics (several times worse than air). : A stainless steel shielded, coaxial cable brings the signal out of the liquid helium to the top of the cryostat. The total resistance of the leads running from the capacitor to the bridge is less than $1 \Omega$. At $60 \mathrm{~Hz}$, this resistance would cause an added dissipation factor of $1 \times 10^{-6}$ in a $2600 \mathrm{pF}$ capacitor. Since the capacitances of all samples measured in this report are substantially less than this, the lead resistance is seen to have a negligible effect on the measurement of dissipation factor:

A typical measurement requires about 5 to 6 liters of liquid helium allowing from 3 to 4 runs per 25 liter supply dewar. The helium will stay in the croystat for several hours, if necessary, although most measurements are completed in less than an hour. The time for the cryostat to go from room temperature to $4.2 \mathrm{~K}$ and back to room temperature is about 20 hours which allows daily runs.

Since the dewars are constructed of glass, no attempt has been made to measure the dissipation factor as a function of increasing helium pressure. Also in these measurements the sample is immersed directly in liquid helium while in the measurements of others ${ }^{4,5}$ the sample is in a vacuum. 


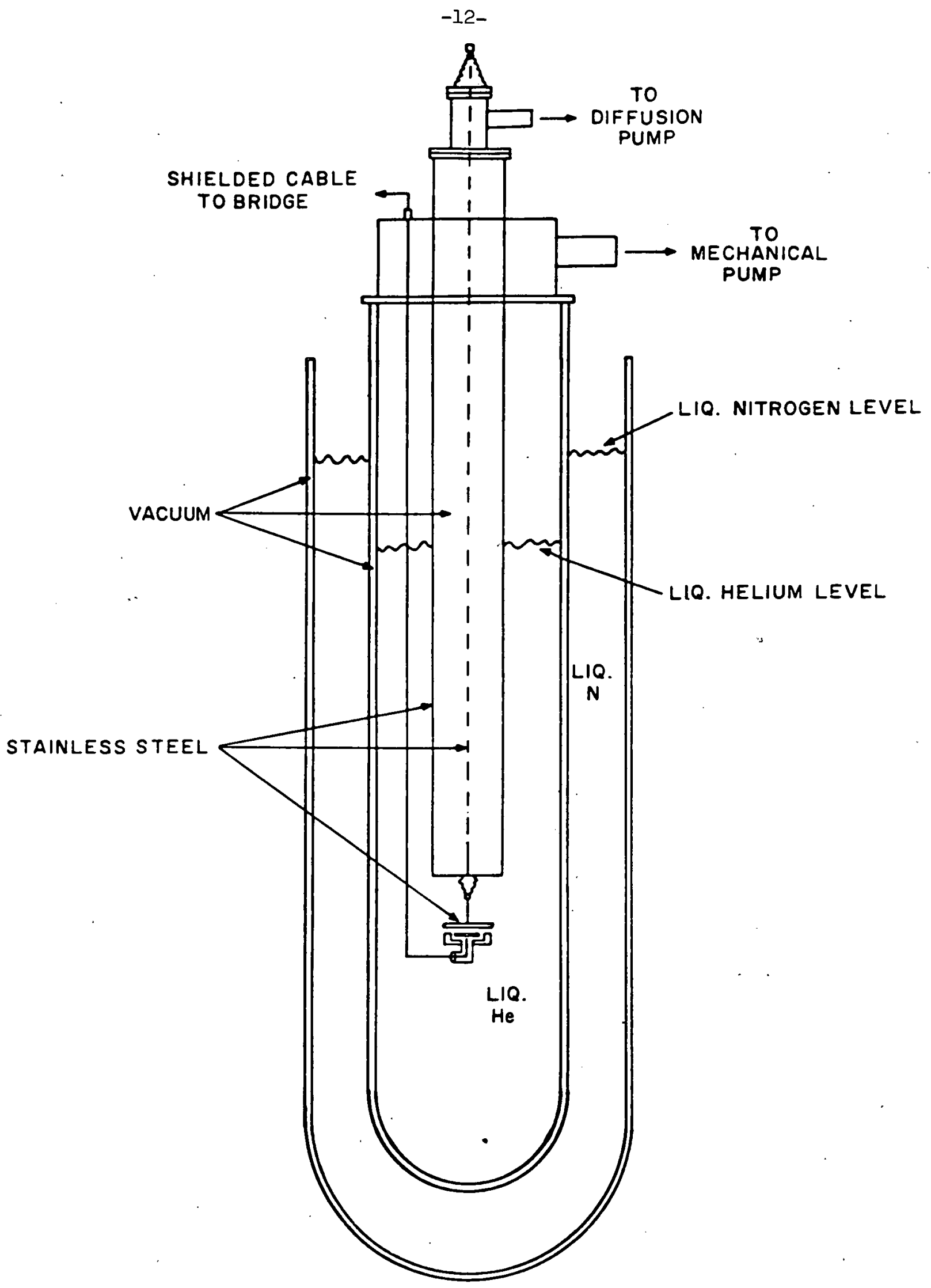

FIG. 3 CRYOSTAT 
Standard Capacitor

Since the dielectric properties of the sample are obtained by electrically balaneing the current in the sample capacitor with the current in the standard capacitor, a knowledge of the properties of the standard capacitor is essential for this measurement. A $100 \mathrm{pF}$ compressed-gas (nitrogen) capacitor is used for the standard. In the majority of high voltage measurements this type of capacitor can be assumed to be lossless. (Commercial capacitors generally claim a dissipation factor of "less than $10 \times 10^{-6 "}$ ). In our measurements, where a total uncertainty of $\pm 1 \times 10^{-6}$ is necessary, the dissipation factor must be accurately measured. The current comparator bridge which will be described in the next. section has the capability of measuring relative dissipation factors to better than $\pm 1 \times 10^{-6}$ but the absolute dissipation factor of the standard must be known in order to determine the dissipation factor of the material being measured. The dissipation factor of our standard capacitor was measured by John Q. Shields of the Absolute Electrical Measurements Section, Electricity Division, NBS. A brief discussion of how this was done ${ }^{6}$ follows.

A guard ring capacitor with variable plate separation is placed in one arm of a transformer ratio arm bridge and the standard capacitor to be measured in the other. Successive relative dissipation factor balances between the variable capacitor and the standard capacitor with auxiliary capacitors in parallel with it are made. Since the relative dissipation factors between the standard capacitor and each of the auxiliary capacitors can readily be measured, then the relative dissipation factor between the standard and the variable-plate capacitor at several settings will be known. If the dissipation factor for the variable plate capacitor is caused only by a surface film, a plot of the relative dissipation factor versus capacitance should be on a straight line as will be shown below. The guard-ring capacitor with electrode spacing, $d$, and film thickness, $t$, is shown in Fig. 4a. This capacitor can be represented by an ideal capacitor, $\mathrm{C}_{1}$, in series with a lossy capacitor with capacitance, $\mathrm{C}_{2}$, and parallel 


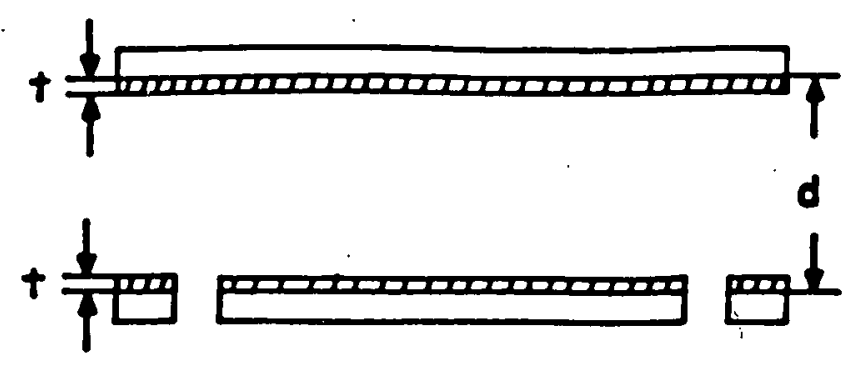

a.

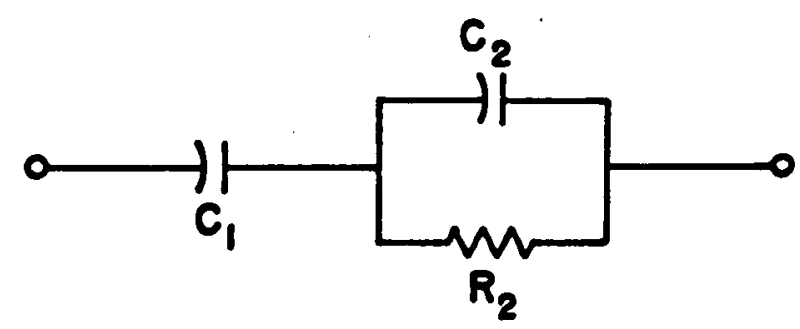

b.

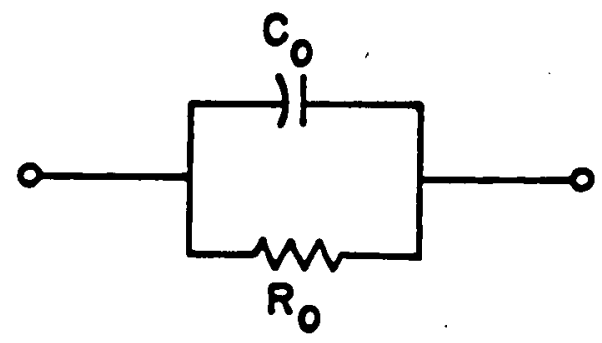

c.

FIG. 4 ELECTRODE FILM CONTRIBUTION TO D.F. 
resistance, $R_{2}$, Fig. 4b. This series combination can be represented as in Fig. $4 c$ by an ideal capacitor, $C_{0}$, in parallel with a resistor, $R_{0}$.

$$
Y_{0}=\frac{Y_{1} Y_{2}}{Y_{1}+Y_{2}}
$$

where

$$
\begin{gathered}
Y_{1}=j \omega C_{1} \\
Y_{2}=1 / R_{2}+j \omega C_{2}
\end{gathered}
$$

and

$$
Y_{0}=1 / R_{0}+j \omega C_{0}
$$

From (4), (5), and (6),

$$
Y_{0}=j \omega C_{1}\left(I+j \omega C_{2} R_{2}\right) /\left(1+j \omega R_{2}\left(C_{1}+C_{2}\right)\right)
$$

or

$$
Y_{0}=\frac{\omega^{2} C_{1}^{2} R_{2}+j \omega C_{1}\left[1+\omega^{2} C_{2} R_{2}^{2}\left(C_{1}+C_{2}\right)\right]}{1+\omega^{2} R_{2}^{2}\left(C_{1}+C_{2}\right)^{2}}
$$

Using (6) and (8)

$$
R_{0}=\frac{1+\omega^{2} R_{2}^{2}\left(C_{1}+c_{2}\right)^{2}}{\omega^{2} C_{1}^{2} R_{2}}
$$

and

$$
C_{0}=C_{1}\left[1+\omega^{2} C_{2} R_{2}^{2}\left(C_{1}+c_{2}\right)\right] /\left[1+\omega^{2} R_{2}^{2}\left(C_{1}+C_{2}\right)^{2}\right] .
$$

The dissipation factor, D.F., is equal to $\left(\omega C_{0} R_{0}\right)^{-1}$.

$$
\text { D.F. }=\omega C_{1} R_{2} /\left[1+\omega^{2} C_{2} R_{2}^{2}\left(C_{1}+C_{2}\right)\right] \text {. }
$$


where

$$
C_{1}=\frac{\varepsilon_{0}^{A}}{d-2 t}
$$

and

$$
C_{2}=\frac{\varepsilon_{r} \varepsilon_{0} A}{2 t}
$$

assuming an area, A, and permittivity $\varepsilon_{r}$ for the film.

$$
\begin{aligned}
& \text { Since } t \ll d \text { or } C_{1} \ll C_{2} \\
& \text { D.F. }=\omega R_{2} \varepsilon_{0} A /\left[(d-2 t)\left(1+\omega^{2} C_{2}{ }^{2} R_{2}^{2}\right)\right]
\end{aligned}
$$

or

$$
D F \approx \frac{K}{d-2 t}
$$

where for a particular frequency $K$ is a constant independent of plate spacing.

The film thickness $t$ is much less than the electrode spacing so (15) can be further approximated

$$
D F \approx K / d
$$

Therefore, if the dissipation factor is caused by surface films, the D.F. should vary inversely with the electrode separation, $d$, and hence be proportional to the capacitance. A plot of relative dissipation factor for the variable capacitor versus capacitance should be on a straight line. The intercept of this line with the dissipation factor axis represents the relative dissipation factor for infinite electrode separation and, therefore, the dissipation factor of the standard capacitor. The fact that the points do lie on a straight line supports this model.

A portable standard capacitor was calibrated in this fashion and found to have a $60-\mathrm{Hz}$ dissipation factor of $-1.1 \times 10^{-6}$ with an uncertainty of $\pm 0.3 \times 10^{-6}$. Our compressed-gas standard capacitor hád a relative dissipation factor of $2.9 \times 10^{-6}$ with respect to this portable standard or an absolute dissipation factor of 
$1.8 \times 10^{-6} \pm 0.5 \times 10^{-6}$

The negative dissipation factor, seemingly worrisome, is easily explained. To see how this is possible, consider Fig. 5. The three-terminal measurement of a guarded lossless capacitor, $C_{S}$, is shown in Fig. 5a. $C_{H}$ and $/ C_{L}$ are the capacitances formed by the guard and the high and low voltage electrodes. In a bridge measurement, the low voltage side is grounded. In this case, the short-circuit transfer admittance of the circuit shown in Fig. $5 \mathrm{a}$ is $\mathrm{y}_{12}=j \omega \mathrm{C}_{\mathrm{s}}$. Now suppose, as in Fig. $5 \mathrm{~b}$, the capacitor's construction is flawed by loss in a common ground return. This frequencydependent mechanism is represented by a resistance, $R$. If $I / R \omega$ is large, compared to both $\mathrm{C}_{\mathrm{H}}$ and $\mathrm{C}_{\mathrm{L}}$, then $\mathrm{y}_{12} \approx j \omega \mathrm{C}_{\mathrm{S}}-\omega^{2} \mathrm{R} \mathrm{C}_{\mathrm{H}} \mathrm{C}_{\mathrm{L}}$. The right hand side of this relation differs from the ideal case by containing a small term which is $\pi / 2$ radians out of phase with $j \omega C_{s} \cdot$ Since the measurement of $y_{12}$ is made on a "black box", the results of the measurement could be caused either by the circuit in Fig. $5 b$ or Fig. 5c. Fig. $5 \mathrm{c}$ is the schematic representation of a guarded capacitor with capacitance $\mathrm{C}_{\mathrm{S}}$ and dissipation factor equal to $-1 / \omega C_{S} R^{\prime}$ where $R^{\prime}=\left(\omega^{2} R C_{H} C_{L}\right)^{-1}$. The dissipation factor is negative. In the case of our portable standard capacitor, the negative dissipation factor was caused by the unsuspected presence of a layer of a high-loss adhesive between the guard and ground.

Current Comparator Bridge

The real part of the dielectric constant and the dissipation factor of a sample are measured by balancing the current in the sample capacitor against the current in the standard capacitor using the current comparator bridge. A simplified version of how this is done is illustrated in Fig. 6. The lossy sample capacitor is represented by a parallel combination of an ideal capacitor, $C_{X}$, and a resistor, $R_{X}$. At balance (i.e., zero signal at the detector, D) since $I_{X} N_{X}=I_{S} N_{S}$,

$$
\mathrm{C}_{\mathrm{X}}=\mathrm{n}_{\mathrm{SD}} \mathrm{n}_{\mathrm{DX}} \mathrm{C}_{\mathrm{X}}
$$

and 

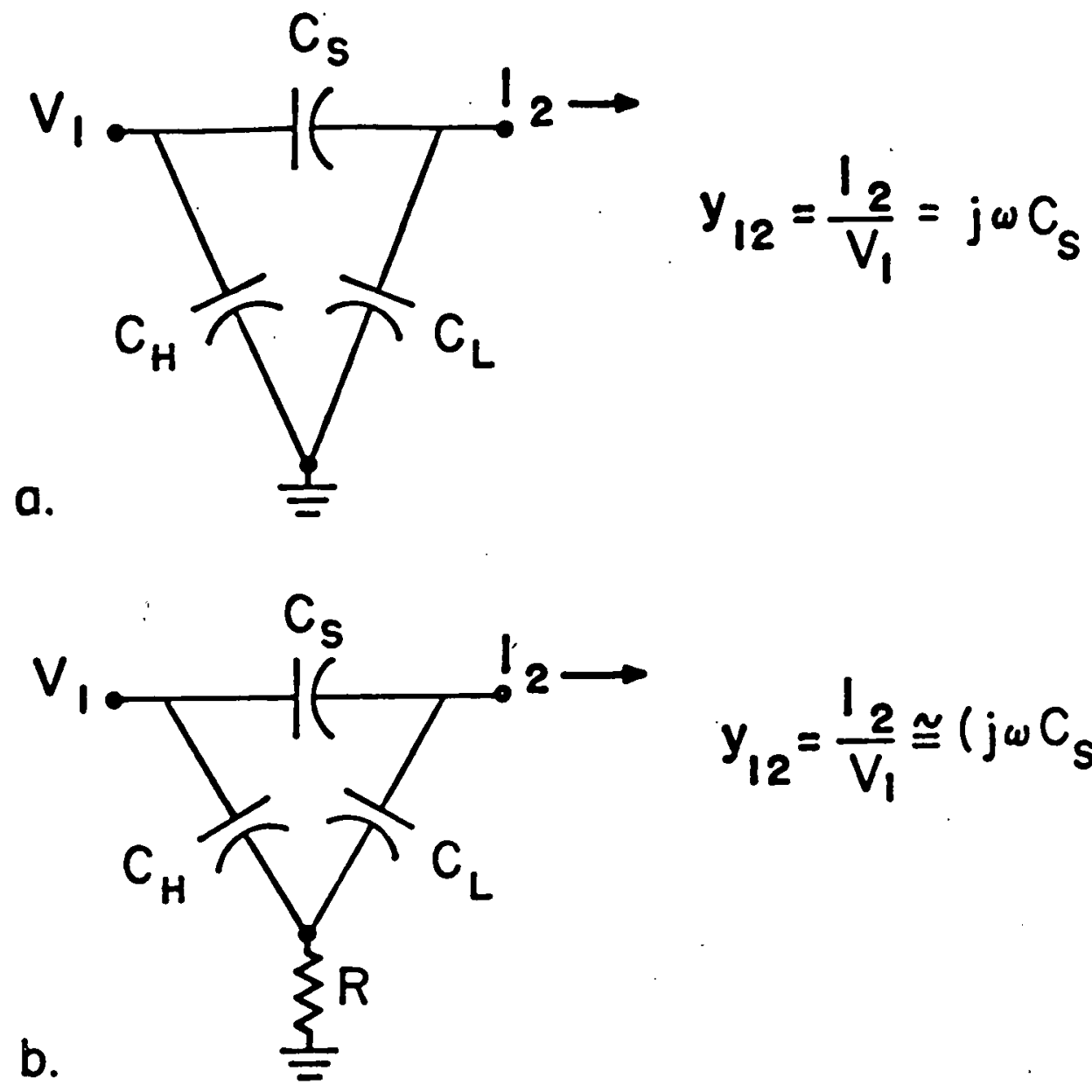

$$
y_{12}=\frac{I_{2}}{V_{1}} \cong\left(j \omega C_{s}-\omega^{2} R C_{L} C_{H}\right)
$$

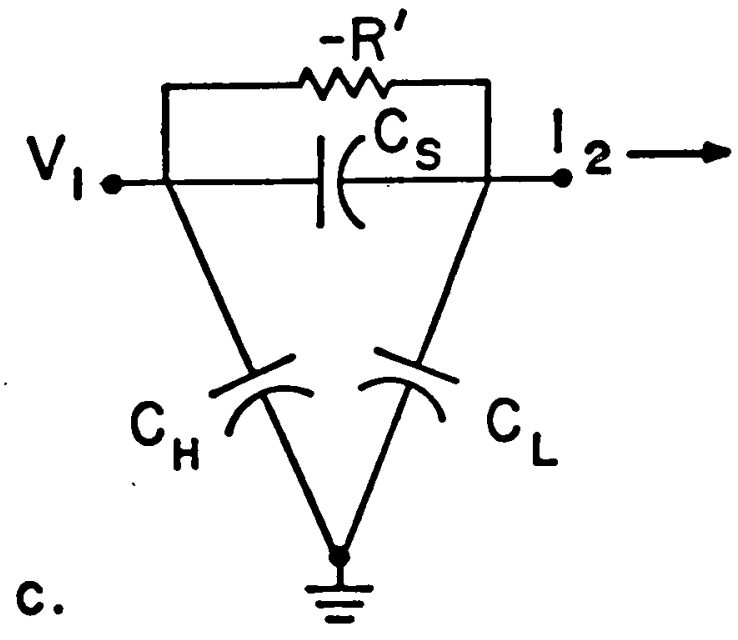

$$
R^{\prime} \cong\left(\omega^{2} R C_{L} C_{H}\right)^{-1}
$$

FIG.5 NEGATIVE D.F. MODEL 


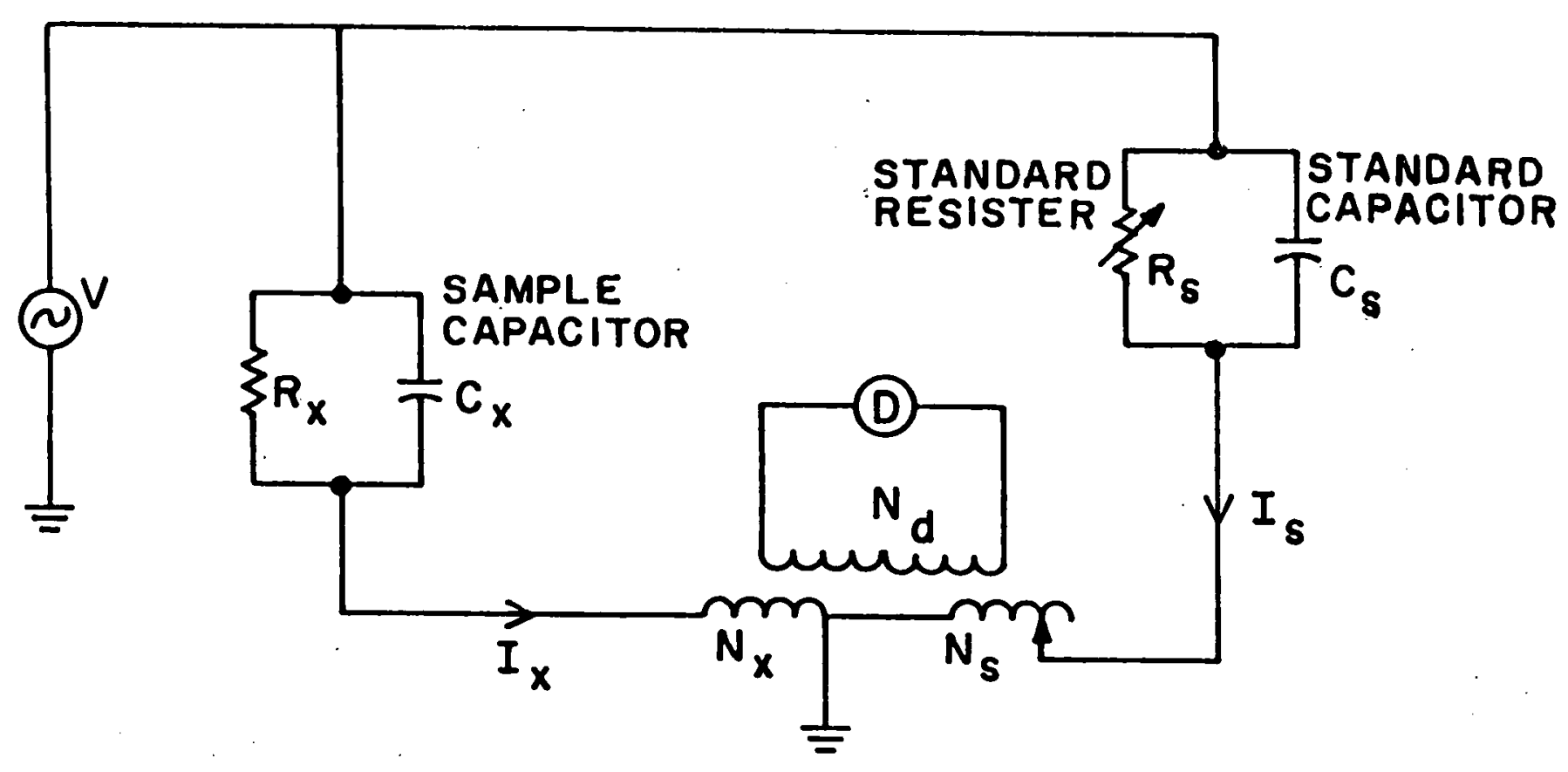

FIG. 6 SIMPLIFIED CURRENT COMPARATOR BRIDGE 


$$
D F=\left(\omega R_{S} C_{S}\right)^{-1}
$$

where

$$
\mathrm{n}_{\mathrm{SD}} \equiv \mathrm{N}_{\mathrm{S}} / \mathrm{N}_{\mathrm{D}}
$$

The transformer in Fig. 6 is a three-winding current transformer or a current comparator. Extensive work on this type of bridge has been done at the National Research Council in Canada and at NBS. 7,8

Unfortunately, the bridge in Fig. 6 is not practical because resistance standards for high voltages are impractical to make, and another method for balancing out the in-phase or loss current must be sought.

Thè circuit in Fig. 7 provides a suitable method for balancing the loss component. An operational amplifier, which provides a virtual ground for the current through the $\mathrm{N}_{\mathrm{S}}$ winding (the amplifier's gain being greater than $10^{4}$ ), outputs a voltage signal equal to $-\mathrm{V} \mathrm{C}_{\mathrm{S}} / \mathrm{C}_{\mathrm{F}}$, typically one thousandth of the source voltage, $\mathrm{V}$, or smaller. A variable resistance which is connected between the amplifier output and the third current-carrying ratio winding, $\mathrm{N}_{3}$ (opposite in polarity to $\mathrm{N}_{\mathrm{S}}$ ) is used to balance the loss component. The balance equations now become:

$$
\begin{gathered}
\mathrm{C}_{\mathrm{X}}=\mathrm{n}_{\mathrm{SD}} \mathrm{n}_{\mathrm{DX}} \mathrm{C}_{\mathrm{S}} \\
\text { D.F. }=\left(\mathrm{N}_{3} / \mathrm{N}_{\mathrm{S}}\right)\left(1 / \omega \mathrm{C}_{\mathrm{F}} \mathrm{R}_{\mathrm{S}}\right)
\end{gathered}
$$

In the actual bridge the switches for the $N_{3}$ and $\mathbb{N}_{S}$ windings are mechanically coupled so that the bridge can be made direct reading in dissipation factor for a particular frequency (e.g., $60 \mathrm{~Hz})$.

The resistance of the leads between the voltage source and the capacitors and between the capacitors and the bridge will contribute to the dissipation factor. In the case of the standard capacitor side of the bridge, it would take a lead. resistance of about 25 ohms to cause an apparent decrease in DF of $1 \times 10^{-6}$. On the 


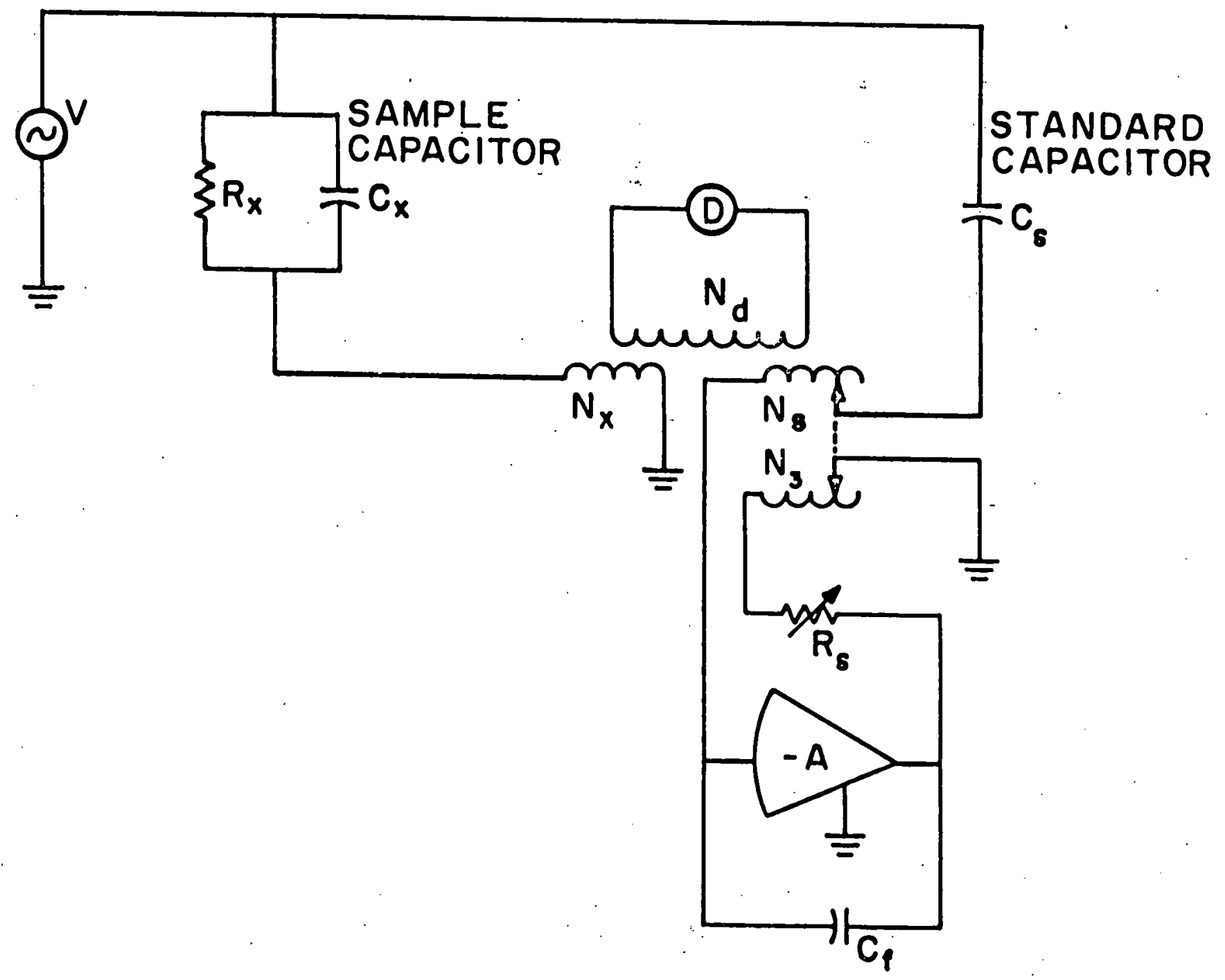

FIG. 7

CURRENT COMPARATOR BRIDGE USING ACTIVE CIRCUITRY FOR D.F. BALANCE 
sample capacitor side of the bridge, however, the lead resistance could become significant. From (2) the capacitance for the sample capacitor could be as large as $1000 \mathrm{pF}$ so that an increase in DF of $1 \times 10^{-6}$ would be caused by a lead resistance of 2 to 3 ohms. The actual lead resistance in our measuring circuit is less than 1 ohm so that the lead resistance is still negligible. Future work on larger capacitance sample holders will require careful consideration of the effect of the leads. The bridge in Fig. 8 remedies this problem by automatically compensating for the lead resistance between the sample capacitor and the bridge. The small voltage, $v$, caused by this lead resistance results in the voltage drop across the sample capacitor being $\mathrm{V}-\mathrm{V}$ instead of $\mathrm{V}$. To compensate for this, the voltage $v$ is inverted and passed through the capacitor, $C_{S}^{\prime}$, the resulting current entering the $\mathrm{N}_{\mathrm{S}}$ winding. The total current in the $\mathrm{N}_{\mathrm{S}}$ winding then becomes

$$
I_{S}=V j \omega C_{S}-v j \omega C_{S}^{\prime}
$$

or

$$
I_{S}=j \omega C_{S}\left(V-v C_{S}^{\prime} / C_{S}\right)
$$

If $C_{S}^{\prime}$ is set equal to $C_{S}$, then

$$
I_{S}=j \omega C_{S}(V-v)
$$

The resulting current is as if a voltage $V-v$ was placed across the standard capacitor, the same voltage that is across the sample capacitor. The compensating circuit, therefore, has eliminated the effect of this lead resistance.

From (2), (20), and (2l) the dielectric-constant, $\varepsilon^{\prime}$, and the dissipation factor, D.F., of the sample can be found from the bridge by the following equations:

$$
\begin{gathered}
\varepsilon^{\prime}=\left(1.08 \times 10^{-4}\right)\left(\mathrm{d} \mathrm{n}_{\mathrm{SD}} \mathrm{n}_{\mathrm{DX}} \mathrm{C}_{\mathrm{S}}\right) \\
\text { D.F. }=\left(\mathrm{N}_{3} / \mathrm{N}_{\mathrm{S}}\right)\left(1 / \omega \mathrm{C}_{\mathrm{F}} \mathrm{R}_{\mathrm{S}}\right)+1.8 \times 10^{-6} .
\end{gathered}
$$




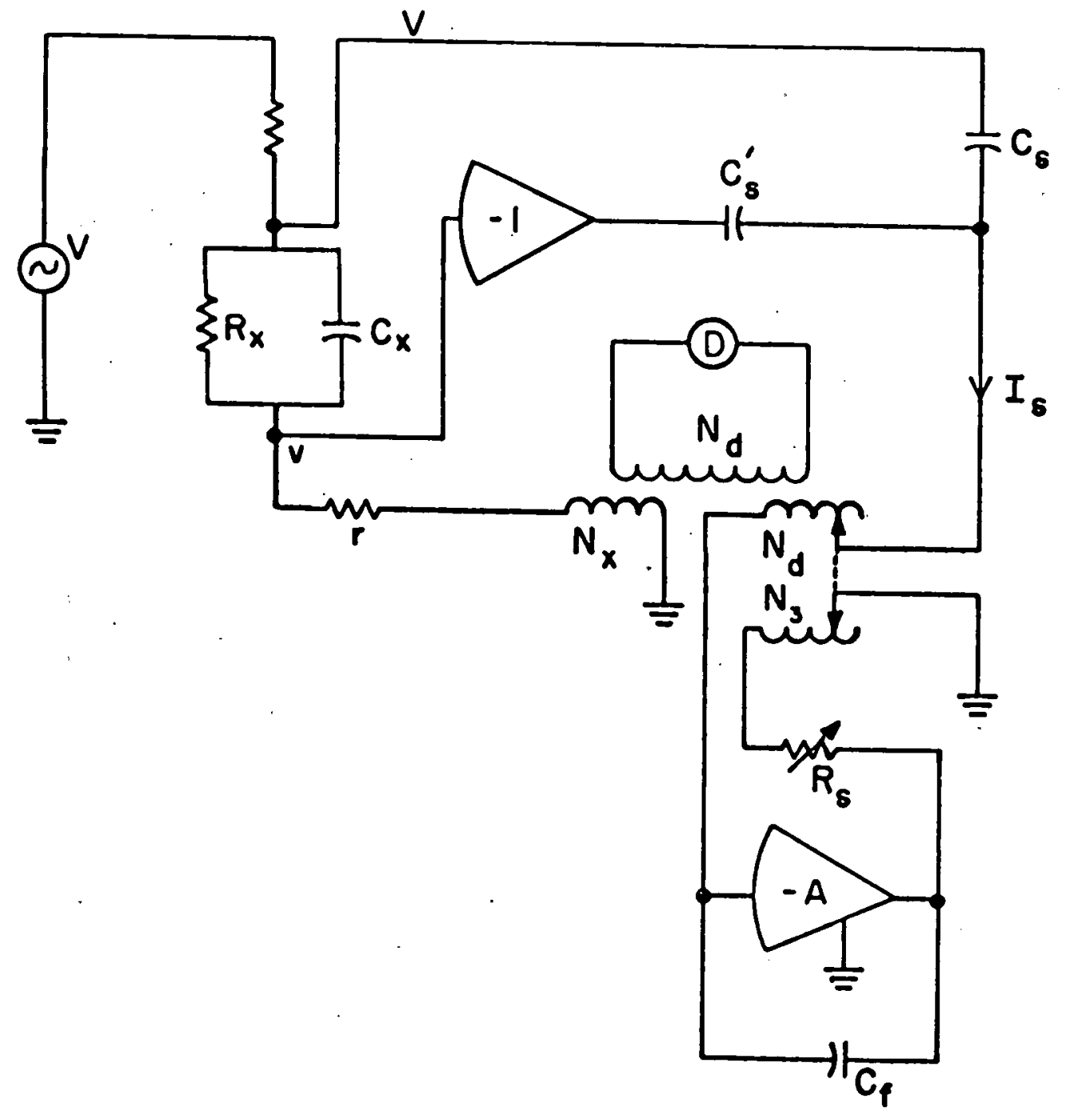

FIG. 8 CURRENT COMPARATOR BRIDGE 
The effect of the dissipation factor of the standard capacitor discussed in the previous section is included in (26).

The estimated uncertainty of (26) is $\pm 1 \times 10^{-6}$ at $60 \mathrm{~Hz}$. The uncertainty in (25), the dielectric constant, may be as large as \pm 10 percent even though the turns ratios, $\mathrm{N}_{\mathrm{SD}}$ and $\mathrm{N}_{\mathrm{DX}}$, are known to $\pm 1 \times 10^{-6}$. This is because the actual electrode spacing at $4.2 \mathrm{~K}$ is not known. In our measurements the room temperature value of $d$ is used thereby assuming no thermal contraction of the sample. The thickness of these thin samples is not always uniform which also contributes to the error. Errors due to coating the sample with paraffin oil (discussed below) are negligible.

\section{RESULTS}

The equipment described previously was used to measure samples of polymer tapes and epoxies submitted by BNL, ORNL (Oak Ridge National Laboratory), and UCC-Linde. As far as the polymer tapes were concerned, only those that proved to be among the most promising from mechanical tests, low-voltage electrical tests, or economic

considerations were sent to us by BNL. Each sample was measured at least twice to test repeatability and prevent erroneous values caused by the occasional cracking of some sample materials at cryogenic temperatures. All values reported are at $4.2 \mathrm{~K}$ and atmospheric pressure, and all measurements were made with the material actually submerged in liquid helium. The voltage values reported below are the root-mean-square values of the applied $60-\mathrm{Hz}$ voltage.

As a consistency check we measured the dissipation factor of liquid helium at $4.2 \mathrm{~K}$. This was accomplished by placing three evenly spaced circular pieces ( 1 mm diameter) of polycarbonate on the guard ring to separate the active electrode from the high voltage electrode. The resultant electrode spacing was $75 \mu \mathrm{m}$. The liquid helium filled capacitor was somewhat noisy at elevated electric stress. At $1.5 \mathrm{kV} / \mathrm{mm}$ the noise was absent and a measurement yields a $60 \mathrm{~Hz}$ dissipation factor of $(+0.3 \pm 0.7) \times 10^{-6}$. This is consistent with the fact that liquid helium has no intrinsic loss mechanisms at power frequencies. 
The results of measurements on single layers of insulators are shown in Iables I and II. The voltage dependence of the dissipation factor is in most cases quite small. Measurements were terminated at the first signs of corona onset as evidenced by noise spikes on the detector output. The repeatability of polymeric samples from the same manufacturer is generaliy good although polymers with the same generic name may differ markedly.

In private conversations, with Mopsik of the Polymers Division, NBS, and King and Thomas of Stanford University we found good agreement in the dissipation factor data at $4.2 \mathrm{~K}$ and $60 \mathrm{~Hz}$.

General comments can be made about the different polymer films listed in Table I. The polyethylenes and polypropylenes (similar in chemical structure) both have dissipation factors considerably less than $30 \times 10^{-6}$ at design stresses of the order of $20 \mathrm{kV} / \mathrm{mm}$. These films along with the polyamide sample, however, have poor mechanical properties at liquid helium temperatures. The polycarbonate and polysulfone films are much better mechanically but have dissipation factors in the range of 50 to $100 \times 10^{-6}$. An attempt by the manufacturer to improve the dissipation factor of one of these materials (green polysulfone) by reducing the concentration of sodium impurities was somewhat successful.

While the dissipation factor of most of the materials increases slightly with increasing voltage, the two polyether-sulfone samples have a negative voltage coefficient. This is similar to the observed behavior of power-factor correction capacitors that use oil-impregnated paper insulation. The effect in that case, according to Garton ${ }^{10}$, is due to the trapping of the mobile ions before the half-cycle is completed. Whether a similar mechanism is causing this behavior in polyethersulfone is not known to us. Since several of the polyether-sulfone films cracked during our measurements, the mechanical properties of this material are evidently poor.

The results of the epoxy measurements are presented in Table II. The epoxies had a nonnegligible voltage coefficient so that the dissipation factors listed are 
Table I. Summary of Results at $60 \mathrm{~Hz}$ and $4.2 \mathrm{~K}$

\begin{abstract}
Material 9
Polyamide (Nylon 11-A; non-oriented).
\end{abstract}

Polycarbonate-A

(uniaxially oriented)

Polyethylene-D

(biaxially oriented, cross

laminated)

Polyethylene- $B$

(biaxially oriented)

\section{Polypropylene-C}

(non-oriented)

Polypropylene-A

(biaxially oriented)

\section{Polypropylene-B}

(biaxially oriented)

Polysulfone-B (clear)

Polysulfone-c (blue).

Polysulfone-D (green)

Polysulfone-E (green,

fewer Na impurities)

Polyether-sulfone-A

(biaxially oriented)

Polyether-sulfone-B

(non-oriented)

$\frac{\text { Thickness }}{40 \mu \mathrm{m}} \quad \frac{\begin{array}{c}\text { Maximum Voltage (Stress) } \\ \text { Applied }\end{array}}{1000 \mathrm{~V} \mathrm{rms}(25 \mathrm{kV} / \mathrm{mm})}$

$75 \quad 2000$

100

40

125

30

40

100

140

140

110

10

30

(20)

2000

800

(20)

2000

(16)

1200

(40)

1200

(30)

1000

(10)

3000

(21)

1200

(9)

1000

(9)

400

(4.0)

800

(27) Maximum Voltage
Tan $\delta$ at

$27 \times 10^{-6}$

69

Tan $\delta$ at $200 \mathrm{~V} \mathrm{rms}$

$24 \times 10^{-6}$

61

6.5

7

3

24

26

15.5

17.5

100

96

126

113

110

105

82

79

28

62

70 
Table II. Summáry of Epoxy Results at $60 \mathrm{~Hz}$ and $4.2 \mathrm{~K}$

Material $^{9}$

Epoxy A, 1.6-mm thick

Epoxy B, 2.8-mm thick

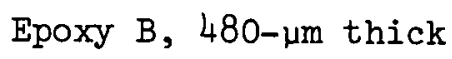

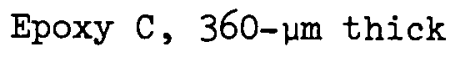

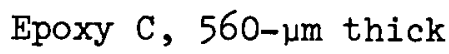

Epoxy C, 630-um thick
$\operatorname{Tan} \delta$

$560 \times 10^{-6}$

340

280

185

210

210 . 
the extrapolated values for zero voltage. The resulting estimated uncertainties for these epoxy measurements are $\pm 5 \%$ of the tabulated value. Sample preparation (including catalyst concentration) may account for the varying results on identical materials of differing thicknesses. The apparent increase of the dissipation factor with sample thickness may be a real effect resulting from the increased probability of voids or other defects. The maximum dissipation factor acceptable for the spacers in the UCC-Linde SPTL ${ }^{11}$ is about $500 \times 10^{-6}$.

In order to make these measurements, it was necessary to coat the electrodesample interfaces with paraffin oil. Presumably this procedure suppresses corona at the interfaces. Measurements by King and Thomas and by us have placed an upper limit of $1 \times 10^{-6}$ on the dissipation factor of paraffin oil at $60 \mathrm{~Hz}$. It has been our experience that when our samples have not been coated with paraffin oil prior to insertion into the sample capacitor, the measured dissipation factor exhibits a strong voltage coefficient. Perhaps this, in part, is caused by the presence of helium bubbles. The corona might be suppressed in supercritical helium.

To examine further the effects of paraffin oil on various interfaces, we studied several stacking arrangements of $30 \mu \mathrm{m}$-thick polypropylene films. The dissipation factor of this material has a very small voltage coefficient over a wide range of voltage stress. We measured the dissipation factor of three-sheet stacks of this material. In one case both sides of each sheet were coated with paraffin oil. In another instance only the metal-insulator interfaces were coated. We also measured a two-sheet stack with oil only between the metal-insulator interfaces.

The results are plotted in Fig. 9. The slopes are consistent with measurements made on polyethylene using the calorimetric method ${ }^{12}$ but it is clear that the exact nature of the interfaces plays an important role. Present designs call for the insulation of a flexible superconducting cable to be impregnated with helium at temperatures of 7 to $9 \mathrm{~K}$ and pressures from 1 to $1.5 \mathrm{MPa}$ ( 10 to 15 atmospheres). It. is therefore our intention to continue our measurements in an apparatus which wi operate at these conditions. 


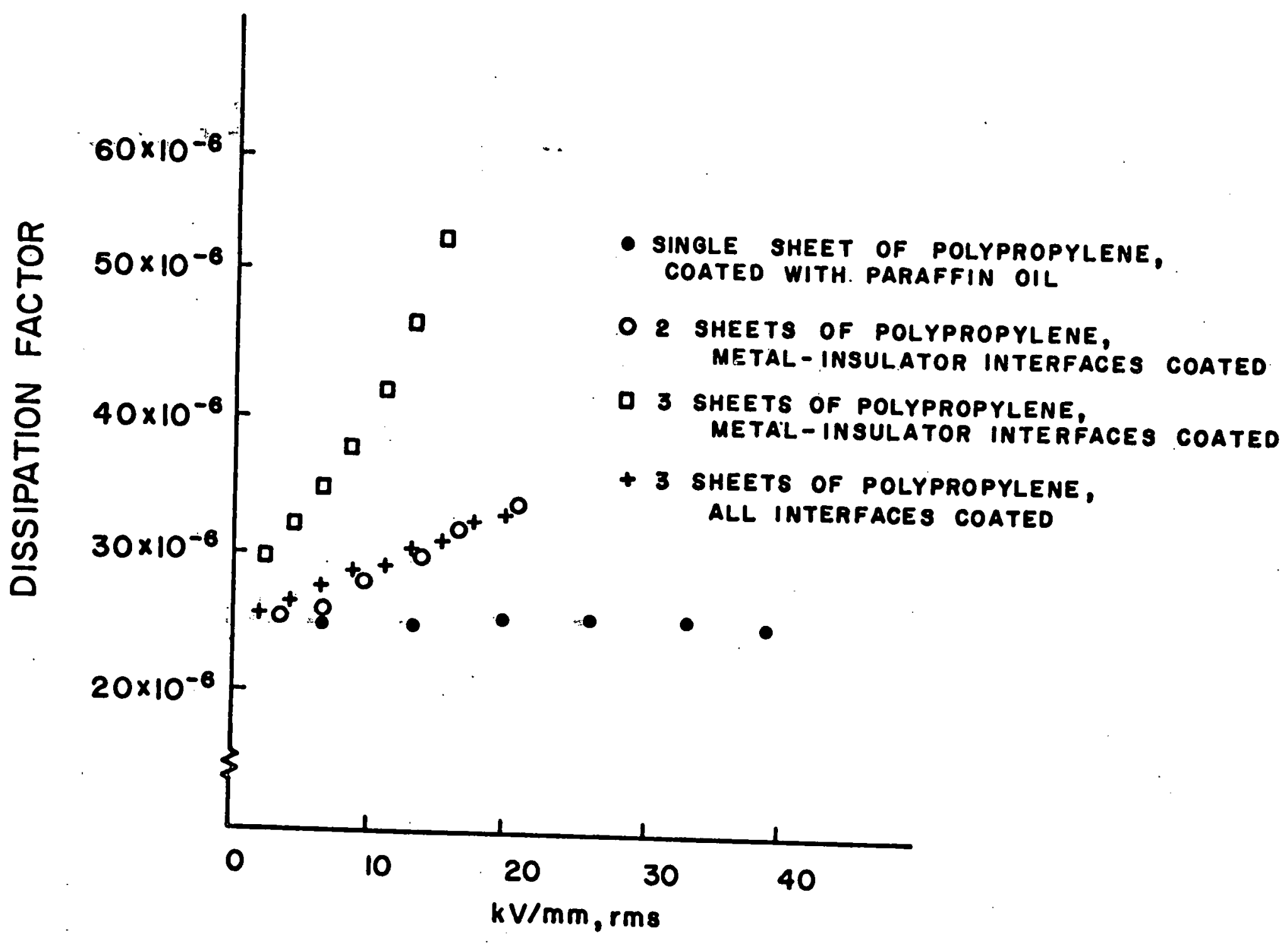

FFIG:TO MULTI-LAYER EFFECT 
WORK IN PROGRESS: CORONA DETECTION SYSTEM

The results reported to date are the outcome of measurements designed primarily to determine dissipation factors in single-layer samples at high electrical stress. The actual design of a flexible AC SPTL calls for wrapped plastic tape insulation impregnated with fluid helium. In such a system, the occurrence of corona between tape layers or in butt gaps may dominate other loss mechanisms. Corona could also prove to be the most important cause of insulation aging in a regime in which "normal" thermal aging is suppressed.

For these reasons we are preparing to study corona losses in simulated cable geometry and environment. The corona detection circuitry can be placed as a shunt across the circuit already in use for dissipation factor measurements (see Fig. 10). In this way, it will be possible to measure dissipation factor and corona simultaneously. The coupling impedance, $\mathrm{Z}$, is an LRC circuit which is driven into oscillation by a partial discharge in the test sample (the other highvoltage circuit. components must be discharge free). The amplitude of the first oscillation is directly proportional to the magnitude of the partial discharge so long as the characteristic period of the LRC circuit is long compared to the rise-time of the partial discharge. The voltage appearing across $\mathrm{Z}$ can be calibrated in picocoulombs by use of a pulse generator in accordance with standard practice. 13,14

For measurement of corona onset, usual practice requires that the signal across $\mathrm{Z}$ undergo amplification and display on an oscilloscope. Narrow-band amplification achieves a low noise level at the expense of a correspondingly limited response time.

Our corona detection apparatus will be designed to sort out partial discharge events as a function of amplitude. These will then be displayed as a histogram of discharge frequency versus magnitude in complete analogy to the pulse height analysis experiments of nuclear physics. The area under the histogram is a measure of the 


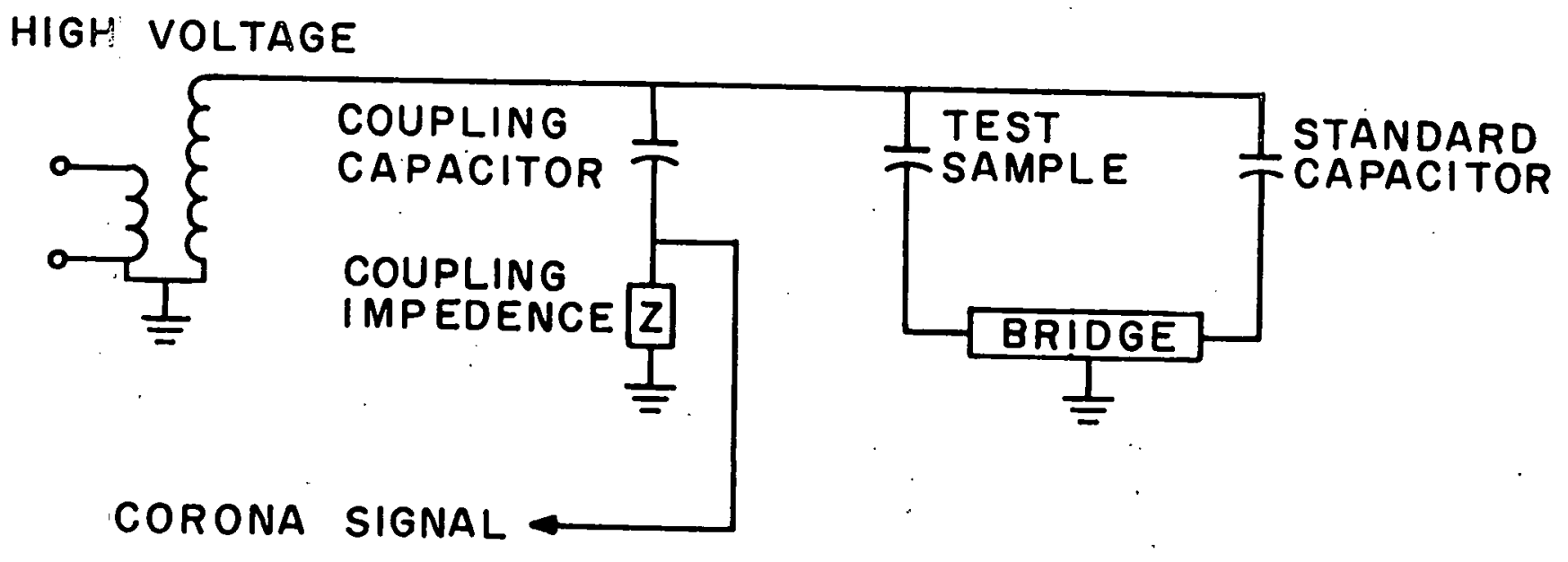

FIG. IO CORONA DETECTION CIRCUIT 
loss energy due to partial discharges. The shape of the histogram should provide an indication as to the source of the discharges. ${ }^{15}$ Aging of the dielectric can be discerned by observing the evolution of histogram features.

Pulse-height analysis in our laboratory will be accomplished using a minicomputer. interfaced to an oscilloscope. Partial discharge events will be detected using the circuit shown in Fig. 10. The magnitude of each event will be converted to a binary number which is then stored and manipulated by the minicomputer. The intervening circuitry between the voltage across $\mathrm{Z}$ in Fig. 10 and the A/D converter has been designed and will now be described.

\section{Electronics}

The detection impedance, $\mathrm{z}$, is an LRC circuit whose characteristic response to an impulse is a critically damped wave whose width is $i_{3} \mu \mathrm{s}$. The rest of the circuitry is shown in Fig. 11. Since the response of the LRC circuit may be of either polarity (depending on the polarity of the partial discharge) the first step is to rectify the output using a diode bridge network. It is recognized that the non-ideality of real diodes will make the output of the bridge somewhat non-linear with voltage but this is not seen as a serious problem.

Next the signal is stepped up by a high-frequency transformer. This transformer accomplishes several things. First, it isolates the output of the diode bridge from ground. Second, it blocks the $60 \mathrm{~Hz}$ carrier signal from the rest of the circuitry. Third, it can be used to step up the signal voltage to a level at which the signal noise is equal to the inherent band-width noise of the succeeding amplifier stage. This voltage transformation will then allow the maximum signal-to-noise ratio to be achieved.

The signal is then amplified by a device with adjustable bandwidth. The bandwidth selected is determined by the various constraints of ability to resolve rapidly occurring events, bandwidth noise, and signal shape. The signal must have a shape which is suitable for the peak-and-hold circuit which follows the amplifier. 


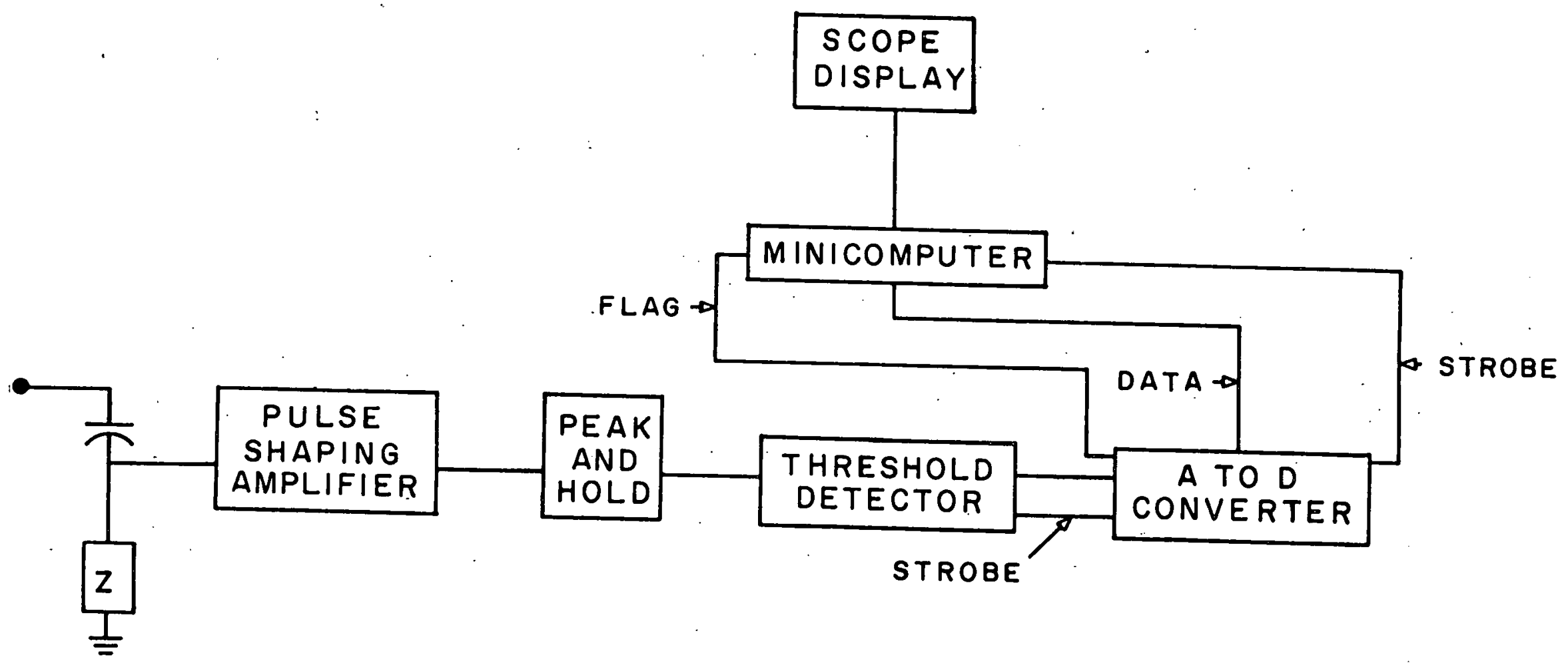

FIG. I SIGNAL PROCESSING 
Fig. 12 shows a schematic drawing of the peak-and-hold circuit we have chosen. When a pulse which has been amplified and shaped appears at the input of $A_{1}$ its peak value will be held at the output of $\mathrm{A}_{2}$. The speed of the peak and hold circuit is determined by the value of the $500 \mathrm{pF}$ capacitor and the current available from $\mathrm{A}_{1}$ :

$$
\frac{d V_{\text {out }}}{d t}=I / C
$$

In our circuit, $C=500 \mathrm{pF}$ and $I=100 \mathrm{~mA}$ so that the slowest peak acquisition time is $500 \mathrm{~ns}$. The droop rate (i.e., rate at which the peak voltage decays) is equal to the leakage current divided by the capacitance. Amplifier $\mathrm{A}_{2}$ has an FET input to keep leakage current below $100 \mathrm{pA}$. This value then gives a maximum droop rate of $0.2 \mathrm{~V} / \mathrm{s}$. This rate is sufficiently slow for data to be transferred to the $A / D$ converter with negligible error.

The comparator in Fig. 12 signals the $A / D$ converter to ignore any signal below $\mathrm{V}_{\mathrm{REF}}$. When the comparator receives a signal, $\mathrm{V}_{\text {out }}$, which exceeds $\mathrm{V}_{\text {ref }}$, it sends a delayed signal to the $A / D$ converter to read $V_{\text {out }}$. The delay is necessary in order to give adequate time for the data pulse to reach its peak. The switch $\mathrm{S}_{2}$ is opened and $\mathrm{S}_{1}$ is closed during the time the $\mathrm{A} / \mathrm{D}$ converter is working.

When conversion is finished, $\mathrm{S}_{3}$ discharges the capacitor, $\mathrm{S}_{1}$ opens, $\mathrm{S}_{2}$ closes and the circuitry is ready to receive another pulse.

\section{Data Analysis}

The high-speed A/D converter (conversion time of $4 \mu \mathrm{s}$ ) will respond to each corona event by sending 12 parallel bits of information to the computer for processing. By using a selector channel input. (direct memory access) the data enters the memory without central processor involvement. This maximizes the rate that data can be entered and allows the minicomputer to be doing other chores.

In order to minimize the dead-time, a two buffer scheme will be used to process the data. While buffer $A$ is being filled with data from the $A / D$, the contents of buffer $B$ are being sorted according to amplitưde and then outputted on an oscilloscope 


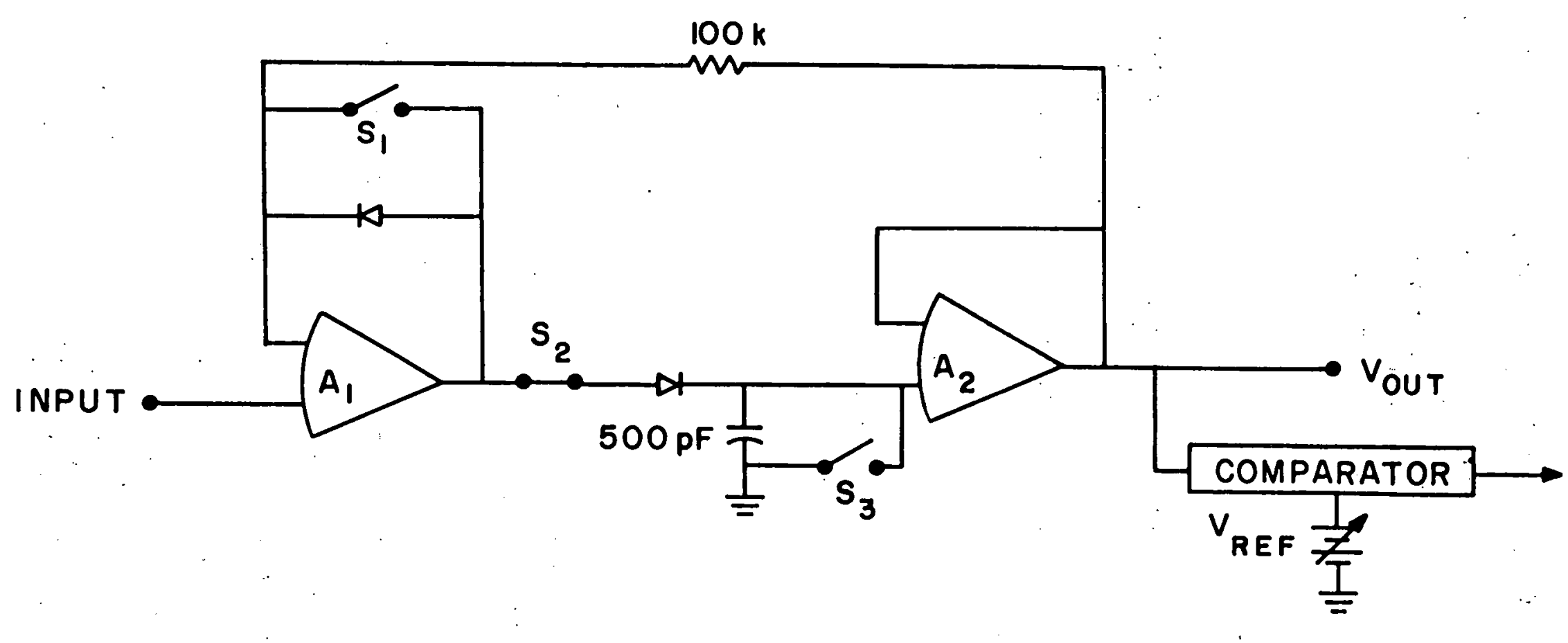

FIG. 12 PEAK-AND-HOLD CIRCUIT 
display. After A is filled, the contents of A are sorted and new data enters B.

After an experiment is completed, a record of the final display will be dumped onto a floppy disc. Each disc contains about 60 tracks usable for data storage. A track contains 32,768 bits--enough storage for one complete experiment. With the use of the floppy discs, different experimental runs can be compared, averaged or otherwise manipulated by the minicomputer.

\section{Progress}

The individual circuits described in the Electronics section have been breadboarded and tested. The operation of the entire circuit, however, has not yet been attempted. The A/D converter. has been interfaced to the minicomputer. Programs (in Assembly Language) need to be written to sort the data and output it to the oscilloscope display.

\section{CONCLUSION}

The dissipation factors of insulating materials being considered for AC SPTL's havè been measured at high electric stress. During the course of this work considerable effort was made to insure accuracy of dissipation factor measurements to $\pm 1 \ddot{x} 10^{-6}$. A current comparator bridge designed at NBS easily provided the needed precision. The required accuracy was achieved by performing a careful determination of the dissipation factor of a compressed-gas standard capacitor. The standard then was balanced against unknown sample dielectrics using the current comparator bridge. As a check of the experimental system, the dissipation factor of liquid helium was measured and found to be less than $1 \times 10^{-6}$ in agreement with theory.

Technical papers describing the experimental technique and the results obtained have been presented at two dielectrics conferences.

, Measurements of stacked layers of plastic--in simulation of the insulation of a flexible cable--suggest that interfacial losses cannot be ignored and, in fact, may predominate. Future efforts will therefore aim at measuring the dissipation 
factor of layers of plastic in a more faithful simulation of cable geometry and environment. Partial discharges occurring in the samples will be studied using pulse-height analysis techniques. 


\section{References}

1. E. B. Forsyth, G. A. Mulligan, J. W. Beck and J. A. Williams, IEEE PAS-94, 161 (1975).

2. G. Bogner and P. Penczynski, Cryogenics, 16, 355 (1976).

3. 1973 Progress Report, part I, BNL Power Transmission Project, p. 87.

4. F. I. Mopsik, 1974 Annual Report, Conf. on Electrical Insulation and Dielectric Phenomena, to be published.

5. C. N. King and R. A. Thomas, 1974 Annual Report, Conf. on Electrical Insulation and Dielectric Phenomena; to be published

6. A. V. Astin, Journal of Research NBS, 22, 673 (1939).

7. N. L. Kusters and 0. Petersons, IEEE CE-82, 606 (1963).

8. 0. Petersons and W. E. Anderson, IEEE IM-24, 336 (1975).

9. Certain commercial materials are identified in order to adequately specify the experimental results. In no case does such identification imply recommendation by the National Bureau of Standards, nor does it imply the material is the best available.

10. C. G. Garton, Proc. IEE 88 II, 103 (1941).

11. Sept. 2, 1975-March 31, 1976 Progress Report, Union Carbide Corporation-Linde Division, p. 9.

12. Semiannual Progress Report for NSF RANN Grant No: GI-3715, p. 10, Oct. 1974.

13. ASTM D-1868-68, 1969 Book of ASTM Standards, part 29, 884 .

14. F. H. Kreuger, Discharge Detection in High Voltage Equipment, Elsevier, N.Y., (1965).

15. R. Bartnikas, IEEE IM-22, 403 (1973). 
Al. Bibliography

The following list of references includes articles which we have found particularly useful. There are four kinds of information represented: general papers on design considerations for superconducting transmission lines, papers dealing specifically with dielectric loss or breakdown at cryogenic temperatures, papers which bear on methods for precise measurement of dissipation factor, and papers describing corona detection systems. To this list must be added the progress reports of other ERDA-supported ac superconducting transmission line research, specifically those of Brookhaven National Laboratory, Stanford University, Oak Ridge National Laboratory and Union Carbide Linde Division.

Allan, R. N. and Kuffel, E., "Dielectric losses in solids at cryogenic temperatures", Proc. IEE 115, 432 (1968).

Astin, A. V., "Nature of energy losses in air capacitors at low frequencies", Journal of Research NBS 22, 673 (1939).

ASTM D-150-68, "Standard methods of test for ac loss characteristics and dielectric constant (permittivity) of solid electrical insulating materials", 1969 Book of ASTM Standards, part 29, 66.

ASTM D-1371-68, "Recommended practice for cleaning specimens for insulation resistance, surface resistance, and volume resistivity testing", ibid., 666.

ASTM D-1868-68, "Detection and measurement of corona pulses in evaluation of insulating systems", ibid., 884 .

Bartnikas, R., "Effect of pulse rise time on the response of corona detectors", IEEE EI-7, 3 (1972).

Bartnikas, R., "Use of a multichannel analyzer for corona pulse-height distribution measurements on cables and other electrical apparatus", IEEE IM-22, 403 (1973).

Baylis, J.A., et al., "AC losses in a composite tubular superconductor for power transmission", Cryogenics 14, 553 (1974).

Belanger, B. C., "Superconducting power transmission programme in the U.S.A." Cryogenics 15, 88 (1975).

Belanger, B. C., "Dielectric problems in the development of resistive cryogenic and superconducting cables", 1973 Annual Report Conf. on Electrical Insulation and Dielectric Phenomena, 486 (1974).

Bobo, J., et al., "Dielectric strength of polymers at cryogenic temperatures under vacuum", Vacuum 18, 397 (1968).

Bogner, G., "Transmission of electrical energy by superconducting cables", NATO Advanced Study Institute, Entreves, Italy, 1973, Superconducting Machines and Devices, S. Foner and B. B. Schwartz, eds, Plenum, 401 (1974). 
Bogner, G., "Cryopower transmission studies in Europe", Cryogenics 15, 79 (1975).

Bosack, D. J., "Development of the dielectric system for a helium cooled superconducting transmission line", 1974 Annual Report Conf. on Electrical Insulation and Dielectric Phenomena, 594 (1975).

Burnier, P. and Moreau, J., "Etude des diélectriques aux temperatures de 1 ! hydrogene liquide", Rev. Gen. I'Elec. $17 ; 600$ (1968).

Carson, R.A.J., "Low-temperature dielectric relaxation in polyethylene", Proc. Roy. Soc. Lond. A 332,255 (1973).

Chant, M. J., "Dielectric properties of some insulating materials over the temperature range 4.2-300 ${ }^{\circ} \mathrm{K}$ ", Cryogenics I, 351 (1967).

Dubois, P., et al., "Research and development of superconducting cables for high power transmission", Proc. 1972 Applied Superconductivity Conf., IEEE Pub. No. 72CH0682-5-TABSC, 173 (1972).

Fallou, B. and Bobo, M., "Electrical properties of insulating materials at cryogenic temperatures", 1973 Annual Report Conf. on Electrical Insulation and Dielectric Phenomena, 514 (1974).

Fallou, B. and Galand, J., "Propriétés de l'hélium liquide", Rev. Gen. l'Elec., I7, 594 (1968).

Gallagher; T. J., "Mobility, conduction and breakdown in cryogenic liquids: a review", 1973 Annual Report Conf. on Electrical Insulation and Dielectric Phenomena, 503 (1974).

Garton, C. G., "Dielectric loss in thin films of insulating liquids", Proc. IEE 88 II, 103 (194I).

Gauster, W. F., et al., "Dielectrics for. superconducting systems", 1973 Annual Report Conf. on Electrical-Insulation and Dielectric Phenomena, 534 (1974).

Gerhold, J.,; "Dielectric breakdown of helium at low temperature", Cryogenics 12, 370 (1972).

Goldschvartz, J. M. and Blaisse, B. S., "Electrical breaktown of liquid helium", Brit. J. Appl. Phys. 17, 1083 (1966):

Horigome, T., "The present state of R\&D for superconducting transmission in Japan", Cryogenics 15 , 91 (1975).

Iwata, Z. and Kikuchi, K., "Electrical insulation for liquid nitrogen cooled EHV cryogenic cable", 1973 Annual Report Conf. on Electrical Insulation and Dielectric Phenomena, 494 (1974).

Jefferies, M. J. and Mathes, K. N., "Insulation systems for cryogenic cable", IEEE PAS-89, 2006. (1970).

Jefferies, M. J. and Mathes, K. N., "Dielectric loss and voltage breakdown in liquid nitrogen and hydrogen", IEEE EI-5, 83 (1970). 
Kreuger, F. H., Discharge Detection in High Voltage Equipment, Elsevier, N.Y., (1965).

Kusters, N. L. and Petersons, 0., "A transformer-ratio-arm bridge for highvoltage capacitance measurements", IEEE CE-82, 606 (1963).

Lampard, D. G., "A new theorem in electrostatics with applications to calculable standards of capacitance", IRE Institution Monograph No. 216M, 271 (1957).

Long, H. M. and Notaro, J., "Design features of ac superconducting cables", J. Appl. Phys., 42, 155 (1971).

Mathes, K. N., "Dielectric properties of cryogenic liquids", IEEE EI-2, 24 (1967).

Mathes, K. N. and McGowan, E. J., "Problems with dielectric measurements at cryogenic temperatures", Measurement of Dielectric Properties Under Space Conditions, ASTM STP $420,3(1967)$.

Meats, R. J., "Pressurized-helium breakdown at very low temperatures", Proc. IEE, $119,760(1972)$.

Meyerhoff, R. W., "AC superconducting power transmission", Proc. 1972 Applied Superconductivity Conf., IEEE Pub. No. 72CH0682-5-TABSC, 194 (1972).

Muller, A. C., "Insulating tape characteristics at cryogenic temperatures", 1973 Annual Report Conf. on Electrical Insulation and Dielectric Phenomena, $524(1974)$.

Nelson, R. L., "Dielectric loss of liquid helium"; Cryogenics 14, 345 (1974).

Nelson, R. L., "Bridge measurement of very low dielectric loss at low temperatures", Proc. IEE 121, 764 (1974).

Petersons, 0. and Anderson, W. E., "A wide-range high-voltage capacitance bridge with one ppm accuracy", IEEE IM-24, 336 (1975).

Phillips, W. A., "Low temperature dielectric relaxation in polyethylene and related hydrocarbon polymers", Proc. Roy. Soc. Lond. A 319, 565 (1970).

Rigby, S. J. and Weedy, B. M., "The effects of semiconducting screens on the dielectric loss of liquid nitrogen impregnated taped cable insulation", Cryogenics 15, 465 (1975).

Rigby, S. J. and Weedy, B. M., "Partial discharges in liquid-nitrogen impregnated, taped-cable insulation", Proc. IEE 123, 165 (1976).

Shields, J. Q., "Phase-angle characteristics of cross capacitors", IEEE IM-2I, 365 (1972).

So, E., "Losses in electrode surface films in gas dielectric capacitors", thesis, 1973, unpublished.

Swan, D. W. and Lewis, T. J., "Influence of electrode surface conditions on the electrical strength of liquified gases", J. of the Electrochem. Soc. 107,.180 (1960). 
$-42-$

Swift, D. A., "Dielectric design criteria for solid-insulated superconducting ac cables", Proc. IEE 118, 1237 (1971).

Swift, D. A., "Dielectric design for a superconducting ac cable with solid insulation", Rev. Gen. 1'Elec. 84, 741 (1975).

Thomas, R. A. and King, C. N., "Low-temperature dielectric loss in polyethylene", . App. Phys. Lett. 26, 406 (1975).

Thompson, A. M. and Lampard, D. G., "A new theorem in electrostatics and its application to calculable standards of capacitance", Nature 177, 888 (1956).

Vincett, P. S., "Colorimetric measurement of very low dielectric loss at low temperatures", Brit. J. Apple. Phys. 2, 699 (1969). 


\section{PART II}

INVESTIGATION IN THE POLYMERS DIVISION ON THE. DIELECTRIC PROPERTIES AND THE

STRUCTURE OF POLYMER TAPES FOR USE AS INSULATION IN SUPERCONDUCTING CABLES

Report Covering Period November 1974 to June 1976 (NBSIR 76-954)

by

F. I. Mopsik and S. J. Kryder (Bulk Properties Section)

and

F. Khoury, J. P. Colson and L. H. Bolz (Structure and Properties Section) 


\section{TABLE OF CONTENTS}

ABSTRACT

p. 45

SUMMARY

1. Dielectric Measurements (F. I. Mopsik and S. J. Kryder)

p. 46

1.1 Introduction

p. 51

1.2 Apparatus and Procedure

p. 51

1.2.1 Ce11

p. 52

1.2.2 Measurement Procedure

p. 52

1.2.3 Measurement Accuracy

p. 56

p. 57

1.3 Results

p. 59

1.3.1 Polycarbonate

p. 59

1.3.2 Nylon 11

p. 61

1.3.3 Polysulfone

p. 61

1.3.4 Polyethylene

p. 63

1.3.5 Polypropylene

p. 68

1.4 Discussion

p. 70

1.5 Summary

p. 72

p. 73

2. Aspects of the Fine Structures of Polymer Tapes (F. Khoury,

J. P. Colson, and L. H. Bolz)

2.1 Introduction

p. 73

2. 2 Crystalline Orientation and Order in the Tapes

p. 75

2.2.1 Background and Experimental

p. 75

2.2.2 Polypropylene

p. 77

2.2.3 Nylon 11

p. 93

2.2.4 Polycarbonate

p. 96

2.2.5 Polyethylene

p. 102

2.2.6 Discussion and Summary

p. 108

2. 3 Some Observations on the Surface Textures of Polymer Tapes

p: 110

2.3.1 Polýpropylene-A Tape

P. 111

2.3.2 Polycarbonate-A Tape

p. 113

2.3.3 Polyethylene-A Tape

p. 115

2.3.4 Summary and Comment

p. 115

p. 117

TABLES

p. 120

GLOSSARY

p. 127 
ABSTRACT

This report is divided into two sections. The first section is on the dielectric properties of the polymer tapes which are being evaluated as insulation for the Brookhaven AC Superconducting Power Transmission Cable Project. In order to measure dielectric loss levels in the tapes a special test cell has been assembled that measures six polymer tape samples at temperatures from $4 \mathrm{~K}$ to $300 \mathrm{~K}$. The loss resolution in $\tan \delta$ is $1 \times 10^{-6}$. Samples of polyethylene, polypropylene, polycarbonate, Nylon 11 and polysulfone have been studied from $4 \mathrm{~K}$ to $273 \mathrm{~K}$. Polyethylene, Polypropylene and Nylon 11 all exhibit a value of tan $\delta$ of $20 \times 10^{-6}$ or less at temperatures of $6-8 \mathrm{~K}$ and $100 \mathrm{~Hz}$ and meet the required dielectric criteria for use in the cables. The presence of antioxidant in polyethylene samples increases $\tan \delta$ below $30 \mathrm{~K}$. The second section of the report is on aspects of the structures of the polymer tapes. In particular, the crystalline orientation in polyethyelene, polypropylene, Nylon 11 and polycarbonate tapes have been surveyed using wide angle $x$-ray diffraction as the main probe. The results are briefly discussed with reference to the mechanical and dielectric properties of the tapes. Some initial observations illustrating differences in surface texture between opposite surfaces in some tapes are also presented. 
SUMMARY

This report on the evaluation of polymer tape insulation for the AC superconducting power transmission cable project at Brookhaven National Laboratory (BNL) is divided into two main sections. Measurements on the dielectric properties of polymer tapes are reported in the first section. Observations on the crystalline orientation and the topography of the surfaces of some tapes are presented in the second section.

\section{Dielectric Properties}

In order to determine the dielectric properties of the polymer tapes, a special test cell has been assembled. The cell can measure the dielectric constant and loss of six samples at the same time at any temperature in the range $4.2 \mathrm{~K}$ to room temperature at audio frequencies. The bridge used with the cell allowed a loss resolution in $\tan \delta$ of $1 \times 10^{-6}$. The materials studied were submitted by BNL and included samples of tapes made from the following polymers: Polycarbonate (4.4'-dioxydipheny 12.2'-propane carbonate), polysulfone, Nylon 11, polyethylene and polypropylene. Most samples were measured over the entire temperature range $4.2 \mathrm{~K}$ to room temperature at frequencies of $100 \mathrm{~Hz}$ and $1 \mathrm{kH}$. The $100 \mathrm{~Hz}$ data are sufficiently representative of the expected behavior at $60 \mathrm{~Hz}$.

The Polycarbonate-A, Polycarbonate- $B$, and Polysulfone-A tapes exhibited excessive loss at $6-8 \mathrm{~K}$ compared to the desired level of $\tan \delta=30 \times 10^{-6}$ for use in the power cable. These tapes had a $\tan \delta$ in excess of $80 \times 10^{-6}$. The Nylon 11-A sample had a $\tan \delta$ of $20 \times 10^{-6}$ at $6-8 \mathrm{~K}$ but poor mechanical properties has ruled out the use of this tape in cables. 
Most of the loss measurements were carried out on polyethylene and polypropylene, which are the main candidates for use in the power cables. Except for a series of polyethylene samples specially prepared for BNL which contained varying amounts of antioxidant and which are discussed separately below, all the polyethylene and polypropylene tapes met the criterion for dielectric loss at 6-8 K. There were some significant differences among these tapes, however. Except for one of the polypropylene tapes (Polypropylene-D) all the polypropylene and polyethylene tapes showed the presence of a loss peak below $4 \mathrm{~K}$. Furthermore, with the exception of three polypropylenes (Polypropylene A, B and D) tan $\delta$ was $10 \times 10^{-6}$ or less at $4-10 \mathrm{~K}$. The loss peak below $4 \mathrm{~K}$ was strong enough in the Polyp ropylene-A and Polypropylene-B tapes to raỉse $\tan \delta$ at $4 \mathrm{~K}$ to $30 \times 10^{-6}$. Polypropylene-D showed a very broad loss peak at $30 \mathrm{~K}$ that caused tan $\delta$ to remain at 20 to $30 \times 10^{-6}$ over the entire $4-10 \mathrm{~K}$ range.

Measurements on a series of polyethylene samples in the form of sheets specially prepared for BNL from polymer to which different amounts of antioxidant were added, showed that the presence of antioxidant in polyethylene can give rise to a loss peak below $4 \mathrm{~K}$. : The loss increased with the amount of antioxidant added to the polyethylene and could be reduced by solvent extraction. The similarity in the dielectric behavior exhibited by the polypropylene and polyethylene tapes indicate that, excess loss observed in the former as the temperature approaches $4 \mathrm{~K}$ is also due to the presence of antioxidant. The only exception seems to be the Polypropylene-D sample where'a different but unknown mechanism seems present. It could not be determined however, whether the additional loss due to antioxidant is related only to its concentration in this tape or whether other factors affect the loss. 
In general the dielectric measurements reported here show that both polyethylene and polypropylene should meet the criterla for dielectric loss at 6-8 $\mathrm{k}$. The possibility exists, however, that as these materials are modified in order to enhance their mechanical properties, the changes in the polymer fine structure could give rise to enhanced dielectric loss either from interactions with any antioxidant present or the appearance of new loss peaks such as the $30 \mathrm{~K}$ loss peak in the Polypropylene-D sample.

\section{Aspects of the Fine Structure of the Polymer Tapes}

In addition to their dielectric properties, an important aspect of the selection of polymer tapes for use as insulation in the superconducting cables is their mechanical properties both at cryogenic temperatures $6-8 \mathrm{~K}$ as well as at room temperature. A vital criterion is the ability of the tapes to withstand the tensions they are subjected to during the tape winding process in cable manufacture. The current guideline used by BNL in this connection is that the tensile modulus of the tapes in the direction of their long axis should be $5 \times 10^{5}-6 \times 10^{5}$ psi (3.4-4.1 GPa) or greater, at room temperature.

The mechanical properties of polymer films are sensitively dependent on the structure of the films which can vary widely with respect to the overall crystallinity of the film, the orientation of the crystallites in the $f i l m$, the manifestation of chain-folding in the crystallites and the occurence of tie molecules between neighboring crystallites. These various features are governed by the conditions under which the polymer crystallizes and the extent to which it is subjected, as is usual in film manufacture, to orientation inducing processes such as uniaxial or biaxial stretching or rolling. In view of the dependance of the mechanical properties of tapes on their fine structures and also because of the possible 
influence of structure on dielectric loss at cryogenic temperatures $(6-8 \mathrm{~K})$, a screening of the respective fine structures of the different tapes was considered desirable as a first step towards establishing guidelines on how their structures could be modified or controlled at the manufacturing stage so as to optimize their mechanical and dielectric properties. A qualitative determination of the nature of the preferred orientation(s) of the crystallites in the tapes was therefore undertaken using $\mathrm{x}$-ray diffraction methods.

Among the features necessary for achieving a high tensile modulus in the direction of the long axis of the tapes are a high crystallinity, and a highly preferred orientation of the crystallites with their constituent chains preferentially parallel to the long axis of the tape. The main observation which emerged in this connection from the x-ray diffraction study of the tapes was that, with the exception of the Polycarbonate-A, all the other tapes, (i.e. the Polycarbonate-B, the Polypropylene-A, B, C, D, E, the Polyethylene-A, D, and the Nylon 11-A) fell short with respect to either crystallinity, or crystallite orientation, or both. It is correspondingly interesting to note from the measurements of the tensile modulus of the tapes at room temperature carried out at BNL, that the present criterion for the tensile modulus was approached closely only by the Polycarbonate-A tape in which there is a distinct preferred orientation of the crystallites with the chain molecules paralle1 to the long axis of the tape. This tape does not, however, meet the present criterion for dielectric loss at 6-8 $\mathrm{K}$, in contrast with the polypropylene, polyethylene; and Nylon 11 tapes which were satisfactory in this respect but are mechanically unsuitable. It may be concluded that new tapes made from polyethylene, polypropylene, and Nylon 11 which have been subjected to high enough 
stretching in the long axis direction during manufacture to impart judiciously high degrees of crystallite orientation with the chain molecules parallel to the long axis of the tape would most probably satisfy the current dielectric and mechanical property criteria for use in the cables. The effects of increased orientation and/or crystallinity on the dielectric properties of such tapes at 6-8 $\mathrm{K}$ would have to be checked, however.

It is not clear at this stage whether differences in dielectric loss levels which were observed among the polypropylene tapes and among the polyethylene tapes examined in the present study are due solely to differences in additive content, or whether structural variations between the tapes were a contribliting factor.

In addition to the examinations of crystallite orientation in the polymer tapes, a.study of another aspect of the structure of the tapes has been initiated, namely the nature of their surface textures. This latter aspect is being investigated in order to obtain insights into the nature of the interfacial contacts between successive windings of the tape in the superconducting cables. The nature of the contacts between successive layers of tape is of interest to BNL in connection with the desired flexibility of the cables, as well as the radial thermal conductivity through the several layers of polymer tape in the cables. Some preliminary light optical and scanning electron microscopical results on three tapes are reported. In particular differences in texture between opposite surfaces of the Polypropylene-A, Polycarbonate-A, and the Polyethylene-A tapes are described. 


\section{Dielectric Measurements (F. I. Mopsik and S. J. Kryder)}

\subsection{Introduction}

The aims of the Polymers Division in establishing a facility for the measurement of dielectric constant and loss at temperatures as low as $4 \mathrm{~K}$ were several. The most important was to determine the dielectric constant and loss at cryogenic temperatures of polymer films that were actively being considered as suitable insulating materials for the Brookhaven Superconducting Cable Project. Another was to provide a facility for testing new materials of possible interest as cryogenic insulation as they become available. Still another was to have enough measurement capability to provide background information on possible sources of dielectric loss.

The electrical requirements for polymer insulating tapes used in superconducting power cables are quite restrictive. They are a dielectric constant, $\varepsilon$, less than 2.5 and a dissipation factor, $\tan \delta$, less than $30 \times 10^{-6}$ at the operating temperatures of the cable, 6-8 $\mathrm{K}$. These requirements in turn present a difficult measurement problem. This is both because of the very low loss levels that have to be determined as well as the form of the samples, films 25-100 $\mu \mathrm{m}$ thick. However, past experience In the Bulk Properties Section, Polymers Division, enabled the design of a suitable sample cell that successfully carried out this measurement task.

These considerations and the emphasis on material properties has led to a facility that allows for the measurement of six samples at the same time for both dielectric constant and loss. The measurements can be made at frequencies in the audio frequency range. The temperature can be maintained conveniently at any temperature from $4.2 \mathrm{~K}$ to room temperature. No attempt was made to work at high applied potentials. This is in contrast to the approach of the Electricity Division where the need for studying the effects of high applied voltage has allowed the measurement of tan $\delta$ for one sample at a time of single samples at $60 \mathrm{~Hz}$ and $4.2 \mathrm{~K}$ only. 


\subsection{Apparatus and Procedure}

\subsubsection{Ce11}

The main problem in establishing the ability to carry out the necessary dielectric measurements was the design and construction of a suitable sample holder. The sample holder, or cell, had to be capable of determining very low losses in thin samples at controllable temperatures. Because of the exploratory nature of the measurements it was felt desirable, in particular, that the cell could be used at any temperáture between $4 \mathrm{~K}$ and room temperature. In addition, to minimize sample handling problems, another desirable feature was the ability to measure more than one sample at a time. The design that was adopted is shown in Figures 1 and 2. The construction of all the necessary parts and the mechanical assembly was done for us by Brookhaven National Laboratory.

The basĭc design consisted of six three-terminal electrode sets put together as a single unit that is surrounded by two sealed cans. The inner can acts as an isothermal shield and the outer can provides the necessary isolation from the cryogenic liquid in which, it is immersed. As a result, six samples could be measured during the same run at any temperature from $4 \mathrm{~K}$ to room temperature with the temperature held constant to about $0.002 \mathrm{~K}$.

The electrode assembly is shown schematically in Figure 1. Six electrodes $1.5 \mathrm{~cm}$ in diameter were cirçularly arranged in the bottom block that acted as the grounded guard. The electrodes were held in place by epoxy cement and beryllium oxide washers for both good electrical isolation and good thermal contact. Six separate leads were brought out to provide-the means to switch from sample to sample. 


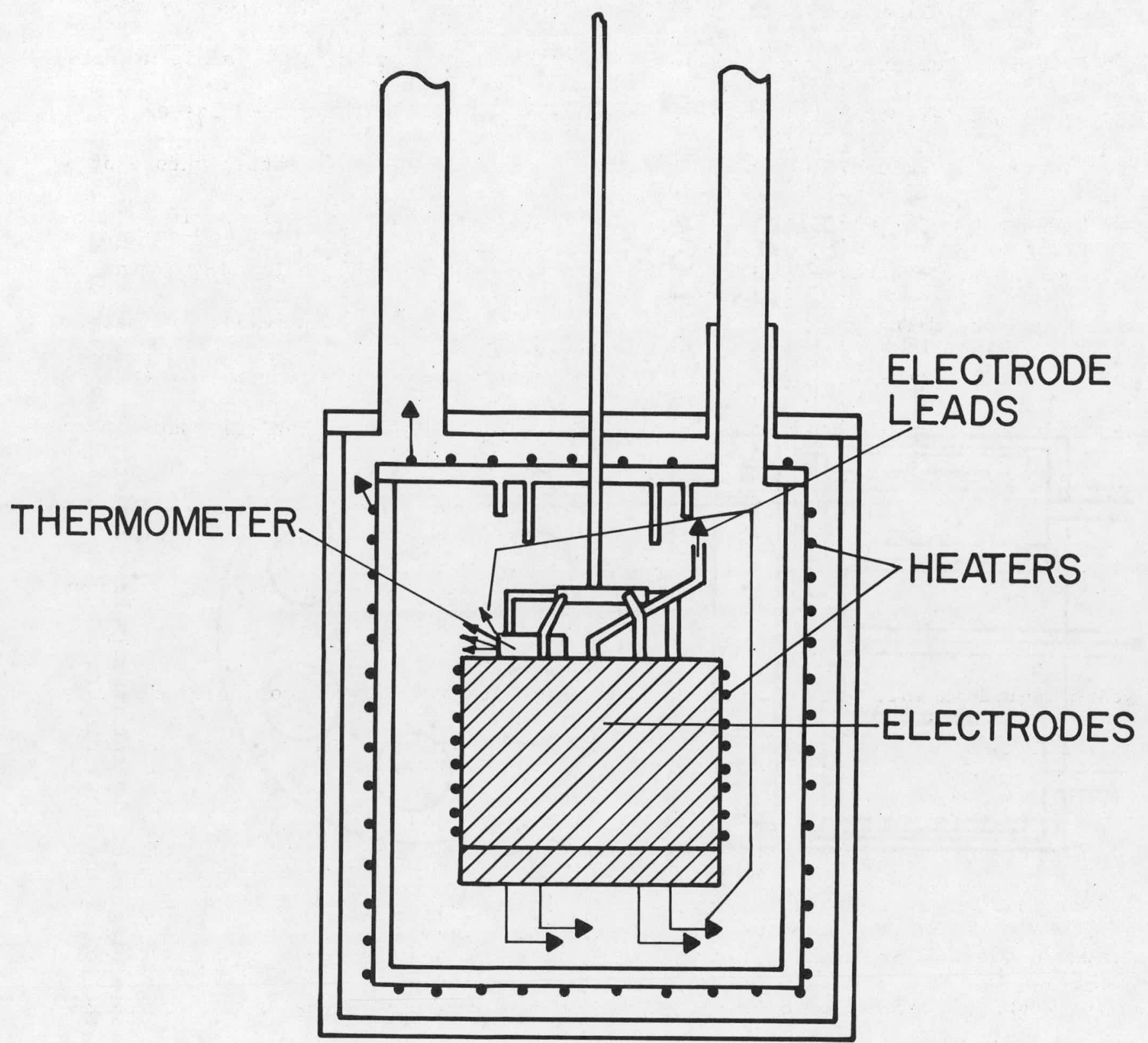

Figure 1: Schematic diagram of the electrode assembly for the cryogenic dielectric ce11. The hatched areas represent insulating spacers. 


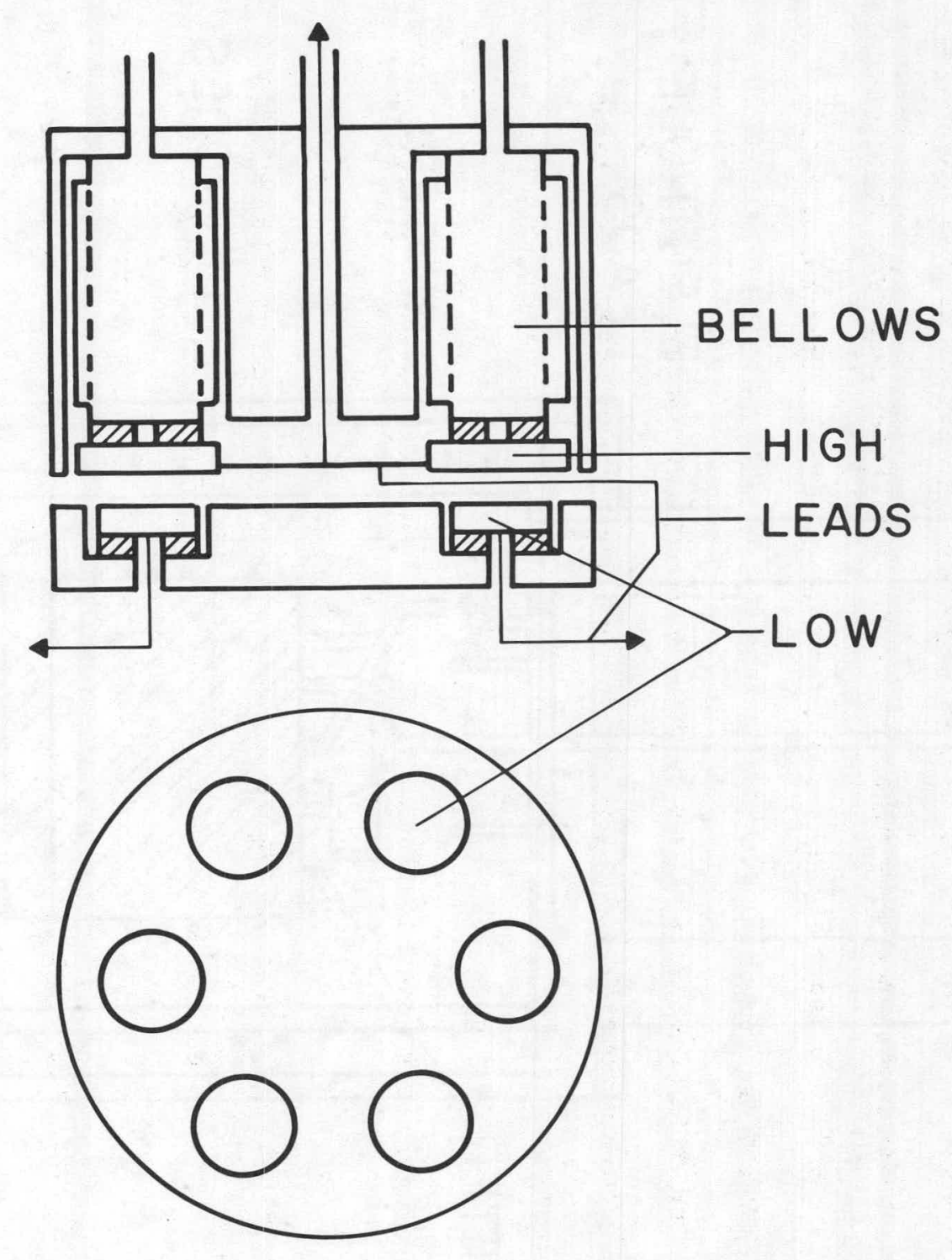

Figure 2: Schematic diagram of the entire cryogenic dielectric cell as it is assembled. The rings projecting from the top of the inner can are used for thermal tempering of all leads going to the electrode assembly. 
After the electrodes were mounted, the entire assembly was ground and laped flat and gold plated. The matching six upper electrodes, $2 \mathrm{~cm}$ in diameter, were individually ground, lapped flat and gold plated. They were attached to bellow assemblies alşo by epoxy cement and beryllium oxide washers. To minimize thermal conduction paths, the upper electrodes shared a common lead. The bellows were connected to a small $(0.3 \mathrm{~cm}$ dia.) stainless steel tube that was the only mechanical tie between the upper block and the top of the inner can from which is was suspended. Apart from electrical leads, the only other contacts to the inner can from the electrode assembly were four loose fitting stainless steel tubes to limit the strain placed on the connecting tube.

Mounted on the upper electrode assembly were an auxiliary heater, a miniature platinum resistance thermometer and a germanium resistance thermometer. All leads going to the electrode assembly were wrapped one turn around tempering rings that were provided on the top lid of the inner can. This was true for the electrode leads as well as the others with care being taken to ensure complete shielding of the lead from the upper electrodes, from the leads of the bottom electrodes.

The entire assembly is shown in Figure 2. The cell and two cans were suspended by two thin-walled stainless steel tubes from the lid of a Dewar that had a $15 \mathrm{~cm}$ inner diameter. The tubes carried the leads from the cell and also served to connect the cans to a gas mainfold and diffusion pump. The tubes were $0.9 \mathrm{~m}$ long and had several baffles attached along their length to minimize convective heat losses in the Dewar. Uniformly wrapped around the inner can, including the top, was a heater proportioned to maintain a constant heating rate on all surfaces. This heater was used as the main heater for controlling temperature of the cell. 
All the leads were brought out on the top of the assembly by vacuum feedthroughs to appropriate connectors. The bottom electrodes were connected to separate coaxial connectors as was the common upper electrode lead. In use, the electrode leads that were not being used for measurement were covered by shorting caps to eliminate the effects of interlead capacitance. A11 the leads were 30 gauge enamel covered wire to minimize thermal conduction except for the electrode leads which were 26 gauge Teflon insulated wire. This heavier gauge wire was used to keep effective lead losses low compared to measurement resolution.

\subsubsection{Measurement Procedure}

Measurements were made by placing six samples $2 \mathrm{~cm}$ in diameter in the cell and sealing the cans in place with indium wire. Both cans were evacuated and back filled with helium gas at a pressure of $200 \mathrm{~mm} \mathrm{Hg}$ at room temperature. When the desired minimum temperature was achieved, the outer can was evacuated and the temperature was controlled. Temperature was monitored above $20 \mathrm{~K}$ by the platinum thermometer and below $20 \mathrm{~K}$ by the germanium thermometer, both of which were calibrated to better than $0.01 \mathrm{~K}$. Both thermometers were supplied a constant current known to $0.001 \%$ and the voltage across them was used both for temperature measurement and control. The current level used in both thermometers gave a sensitivity of about $400 \mu \mathrm{V} / \mathrm{K}$. A three mode proportional controller having a resolution of $1 \mu \mathrm{V}$ and supplying $20 \mathrm{~W}$ of $\mathrm{DC}$ power to the inner can heater was able to keep the temperature constant to within $0.002 \mathrm{~K}$. During the electrical measurements, a pressure of $0.2 \mathrm{MPa}$ ( $2 \mathrm{~atm}$.) of helium was maintained in the bellows to ensure adequate electrode contact. 
The bridge used to determine capacitance and loss was a three-terminal transformer bridge that used substitution. It had a resolution in terms of $\tan \delta$ of $1 \times 10^{-6}$ or better, depending on the sample capacitance. By means of a standard capacitor calibrated for loss by the Electricity Division, the loss uncertainty of the bridge was shown to be $0.5 \times 10^{-6}$ expressed as tan $\delta$. Typical working voltages were $20-40 \mathrm{~V}$.

The useable frequency range of the bridge with the interchangeable transformers that were available was $30 \mathrm{~Hz}$ to $10 \mathrm{kHz}$ but sensitivity and balancing time considerations made a lower frequency of $100 \mathrm{~Hz}$ preferable and lead losses made an upper frequency of several $\mathrm{kHz}$ desirable. In the measurements reported here, only frequencies of $100 \mathrm{~Hz}$ and $1 \mathrm{kHz}$ were used. With the frequency range available, additional frequencies would not have added much additional information and would have lengthened the measurement time excessively. While no data were taken at $60 \mathrm{~Hz}$, since $\tan \delta$ is a slowly varying quantity with respect to the logarithm of frequency, the $100 \mathrm{~Hz}$ data can be used as $60 \mathrm{~Hz}$ data with little extra uncertainty.

\subsubsection{Measurement Accuracy}

The accuracy of $\varepsilon$ and tan $\delta$ for the samples measured and reported here was limited by the samples themselves. The temperature was determined well enough to be considered exact. The bellows pressure chosen was found experimentally to be high enough to get as good electrode contact as possible. Higher pressures were not found to change the results significantly with respect to sample errors. The only requirement on temperature was that it was constant to keep the cell capacitance constant to facilitate bridge balance. Similarly, the bellows system had to be completely tight. At one time a small leak developed in the pressure line that disturbed conditons enough to make measurements difficult. 
Errors associated with the determination of electrode area and the bridge accuracy were negligible. The electrode area, needed to determine $\varepsilon$, was measured with the use of gauge blocks and a reference plane to an accuracy of $0.1 \%$. The bridge had an accuracy of better than $1 \mathrm{ppm}$. The only problem encountered was that sometimes the leads vibrated excessively, leading to increased noise at 100 $\mathrm{Hz}$, which deteriorated resolution, although usually not seriously.

The samples themselves were the main limit in the determination of $\varepsilon$ and $\tan \delta$. One limitation was the determination of sample thickness which enters directly in the computation of $\varepsilon$. Sample thicknesses were measured by micrometer at room temperature to $1 \mu \mathrm{m}$ which is an error of 1 to $5 \%$ depending on sample thickness. It was this thickness value that was used for all temperatures with no correction made for thermal contraction since this data was not generally available to us. The rise in $\varepsilon$ for a material such as polyethylene with decreasing temperature, as reported later, is undoubtedly partly due to this neglect.

When thermal expansion data do become available, the values of $\varepsilon$ should be corrected. Another source of error is the error that results from the films not being perfectly flat and in perfect contact with the electrodes. This source of error was checked by the use of Teflon FEP films of varying thickness and known dielectric constant. The apparent dielectric constant for these films was $3 \%$ low. for $125 \mu \mathrm{m}$ films and $12 \%$ low for $25 \mu \mathrm{m}$ films. These values include the error from using the micrometer and should be considered indicative only since they are dependent on the condition of the film surface. Accordingly, these corrections were not applied to the data. Finally, corrections for the guard gap were not made as they are less than the other incertainties in the measurements. 
Sample conditions could also occassionally cause problems. Sometimes a sample would warp or.include a piece of dust, resulting in an unusually low value. of measured capacitance and often enhanced loss, especially at 100 Hz. In most cases duplicate samples prevented this from being much of a problem and those cases where it was will be indicated.

\section{3 $\quad$ Results}

Al1 the samples described in this report were supplied by Brookhaven National Laboratory. They were used as received after being cut into $2 \mathrm{~cm}$ diameter discs. Dust was excluded as much as possible.

\subsubsection{Polycarbonate}

Two different types of polycarbonate film were measured, Polycarbonate-A and Polycarbonate-B. Both films were about $60 \mathrm{\mu m}$ thick and both were dyed. Polycarbonate-A was yellow-green and the Polycarbonate-B was yellow-brown. Two samples were measured for both films with good agreement between the samples. The data are presented in both Table I and Figure 3.

Both films are characterized by a broad loss peak at about $180 \mathrm{~K}$ at $100 \mathrm{~Hz}$. The peak is broad also in a frequency, sense as can be seen by comparing the $100 \mathrm{~Hz}$ and $1 \mathrm{kHz}$ data. While the loss peak for the Polycarbonate-A film is lower than that of the Polycarbonate-B film, at $30 \mathrm{~K}$ and below both films were identical in terms of loss.

In Figure 3, an inset shows the loss behavior of both films in the cryogenic cable temperature range of 4-10 $\mathrm{K}$. The loss decreases slowly with decreasing temperature with no indication of any loss peaks. Tan $\delta$ changes from $80 \times 10^{-6}$ at $10 \mathrm{~K}$ to $60 \times 10^{-6}$ at $4 \mathrm{~K}$. Compared to the maximum loss criterion for use in 


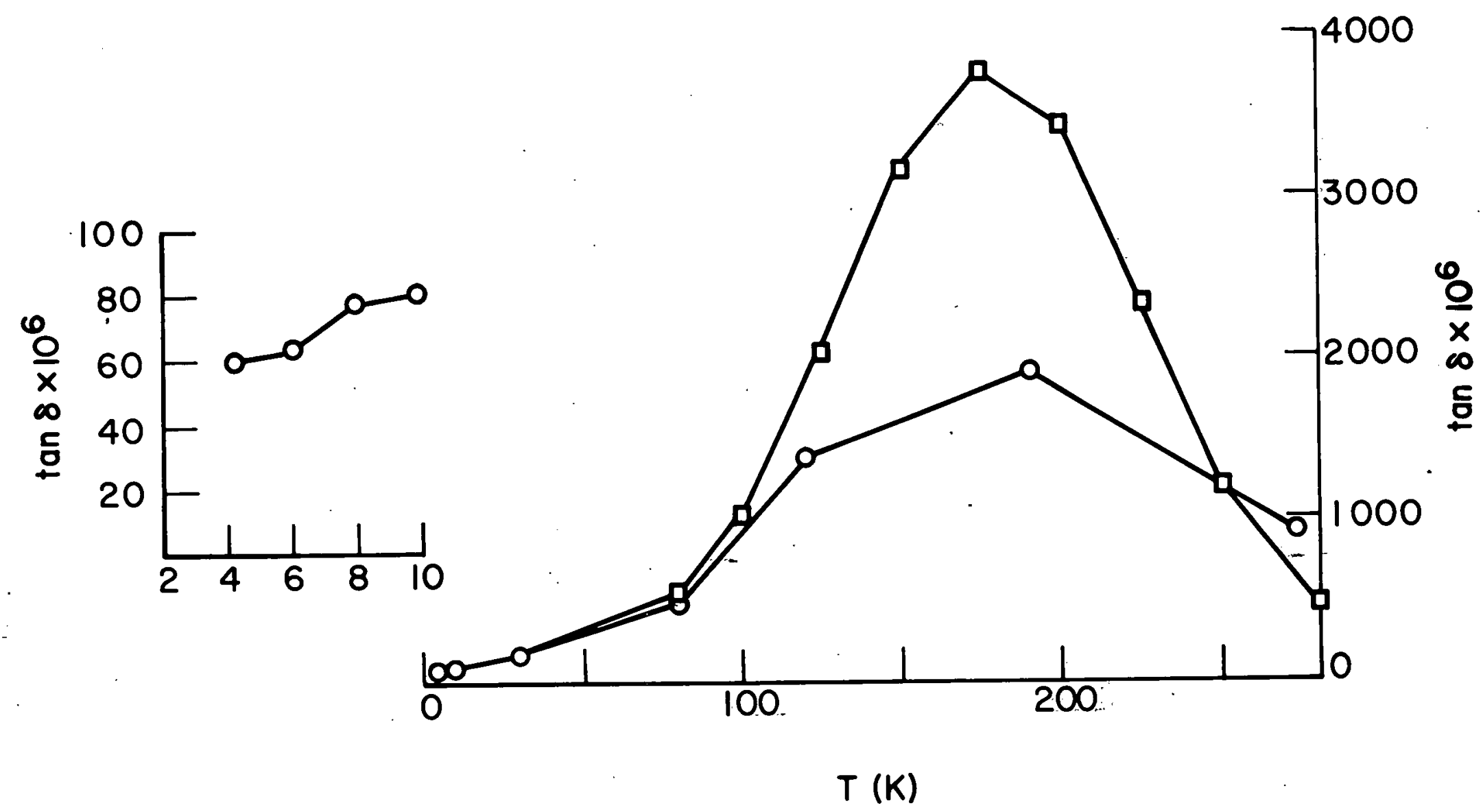

1
8
1

Figure 3: Tan $\delta$ vs. temperature at $100 \mathrm{~Hz}$. The circles are for Polycarbonate-A, the squares for Polycarbonate-B. The inset showing more detailed data below. $10 \mathrm{~K}$ is for both polymers..- 
a power cable of $30 \times 10^{-6}$, both films are definitely not satisfactory. Furthermore, since the $B$ film has poor crystallinity while the A film is distinctly crystalline and exhibits a high degree of crystalline orientation (see Section 2.2.4), structural modifications of these Polycarbonate films do not seem important in modifying dielectric loss at cryogenic temperatures.

\subsubsection{Nylon 11}

Only one type of Nylon $11 \mathrm{film}$, the Nylon 11-A was measured. This was a poorly crystalline sample (see Section 2.2.3). The film was $40 \mu \mathrm{m}$ thick. The data are presented in Table II and Figure 4.

The loss behavior of the Nylon 11-A studied here is quite different from any of the other polymers studied to date. The loss near room temperature is much larger than any of the other polymers but decreases very rapidly with decreasing temperature. By $30 \mathrm{~K}$ tan $\delta$ has become $30 \times 10^{-6}$ and between 4 to $10 \mathrm{~K} \tan \delta$ has become $20 \times 10^{-6}$. With the decrease in loss, the dielectric constant has decreased from 3.0 to 2.5 at cryogenic temperatures. Therefore, in terms of dielectric properties, the Nylon 11 changes from a poor material at room temperature to one that meets the desired criteria at the operating temperature of the superconducting cable.

No further work was done on Nylon 11 due to the unavailability of samples with suitable mechanical properties. However, the data determined here suggests that if a film could be found that is suitable mechanically, it could pe suitable electrically.

\subsubsection{Polysulfone}

Only one type of polysulfone, the Polysulfone-A which is a highly transparent film $50 \mu \mathrm{m}$ thick was measured. The data for this material are shown together with the Nylon 11-A data in Table II and Figure 4. 


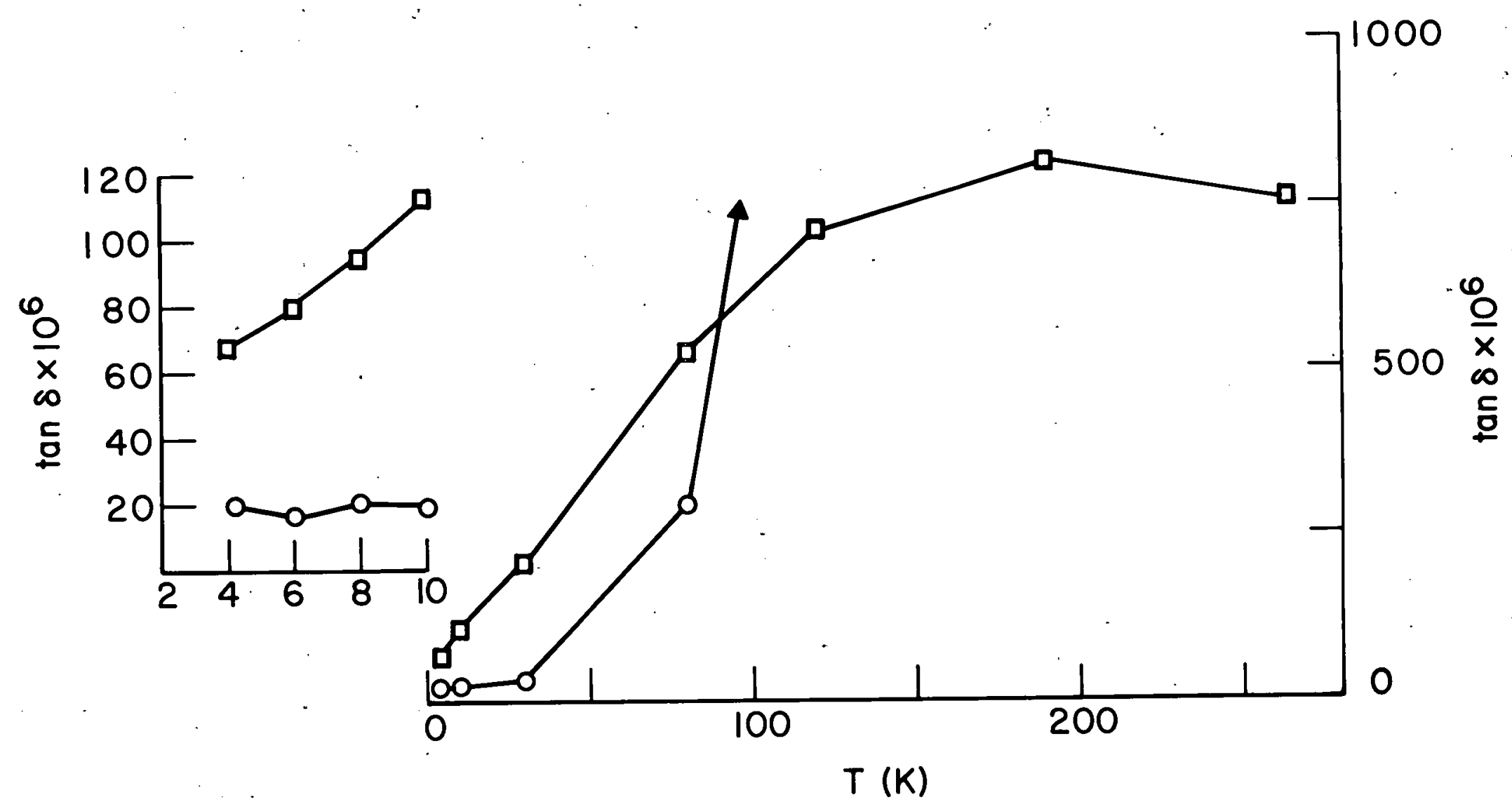

Figure 4: Tan $\delta$ vs. temperature at $100 \mathrm{~Hz}$. The circles are for Nylon 11-A, the squares for Polysulfone-A. 
The loss data show a very broad peak around $200 \mathrm{~K}$ at $100 \mathrm{~Hz}$. The peak is so broad in a temperature sense that it persists down to the lowest temperature attained, $4.2 \mathrm{~K}$. The loss mechanism is also very broad with respect to frequency as seen in the near equality of the $100 \mathrm{~Hz}$ and $1 \mathrm{kHz}$ data (see Table II). Tan $\delta$ varied from 70 to $110 \times 10^{-6}$ in the temperature range of 4.2 to $10 \mathrm{~K}$ which is clearly much too high for the power cable requirements.

\subsubsection{Polyethylene}

A larger number of measurements were made on many different samples of polyethylene. The samples measured include the Polyethylene-A film (26 $\mu \mathrm{m}$ thick) for which data were collected from both single and double layers, the Polyethylene-C and Polyethylene-D films (100 $\mathrm{mm}$ thick), and a series of polyethylene sheets specially prepared for BNL (Polyethylene-BNL, see later and Table III). The data are given in Tables IV and $V$ and Figures 5 and 6. Significant differences in dielectric behavior were found in the different samples of polyethylene. These differences will be pointed out in the following discussion.

The Polyethylene-A was measured both as single layers and double layers. Within experimental accuracy, the data for the double layers were the same as the single layers and, therefore, no separate data will be given for the double layers. This shows that for these films, the presence of an additional interface was not enough to influence the measurements.

At $100 \mathrm{~Hz}$ the loss data for the Polyethylene-A film show a loss peak at about $175 \mathrm{~K}$, a slight rise in loss with temperature beginning at $250 \mathrm{~K}$, and a low leve 1 of loss at all temperatures. The loss at $100 \mathrm{~Hz}$ below $10 \mathrm{~K}$ was constant at $6 \times 10^{-6}$ which is well below the desired maximum. The $1 \mathrm{kHz}$ data do show a slight rise at $4.2 \mathrm{~K}$ but are still quite low. As for all polyethylenes, the dielectric constant is always lower than the desired maximum of 2.5 . 


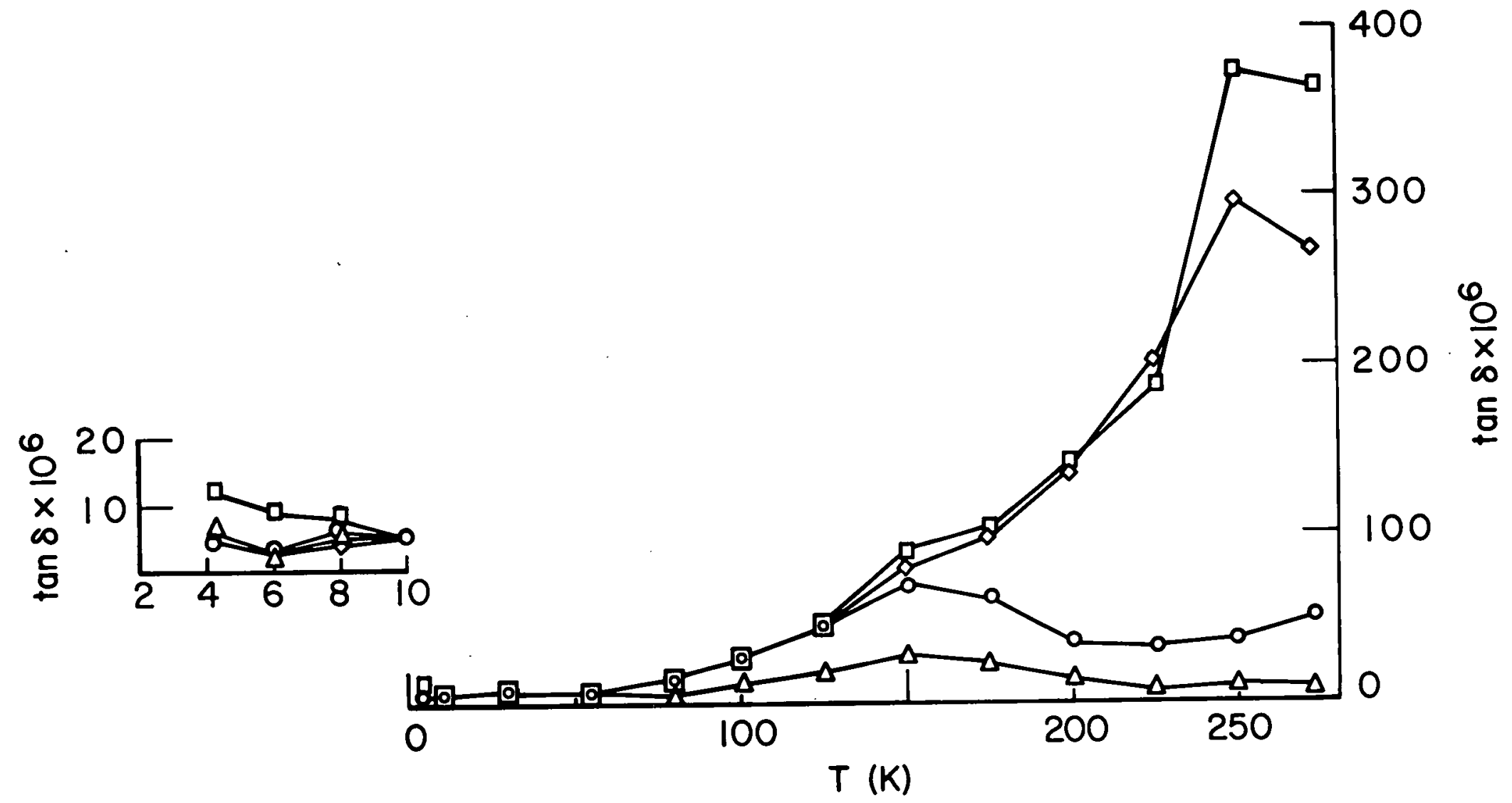

Figure 5: Tan $\delta$ vs. temperature at $100 \mathrm{~Hz}$ for various polyethylenes. Circles represent Polyethylene-A, diamonds Polyethy lene-C, squares Polyethylene-D, triangles Polyethylene-BNL-2. The circle inside the square symbol represents overlapping data. 


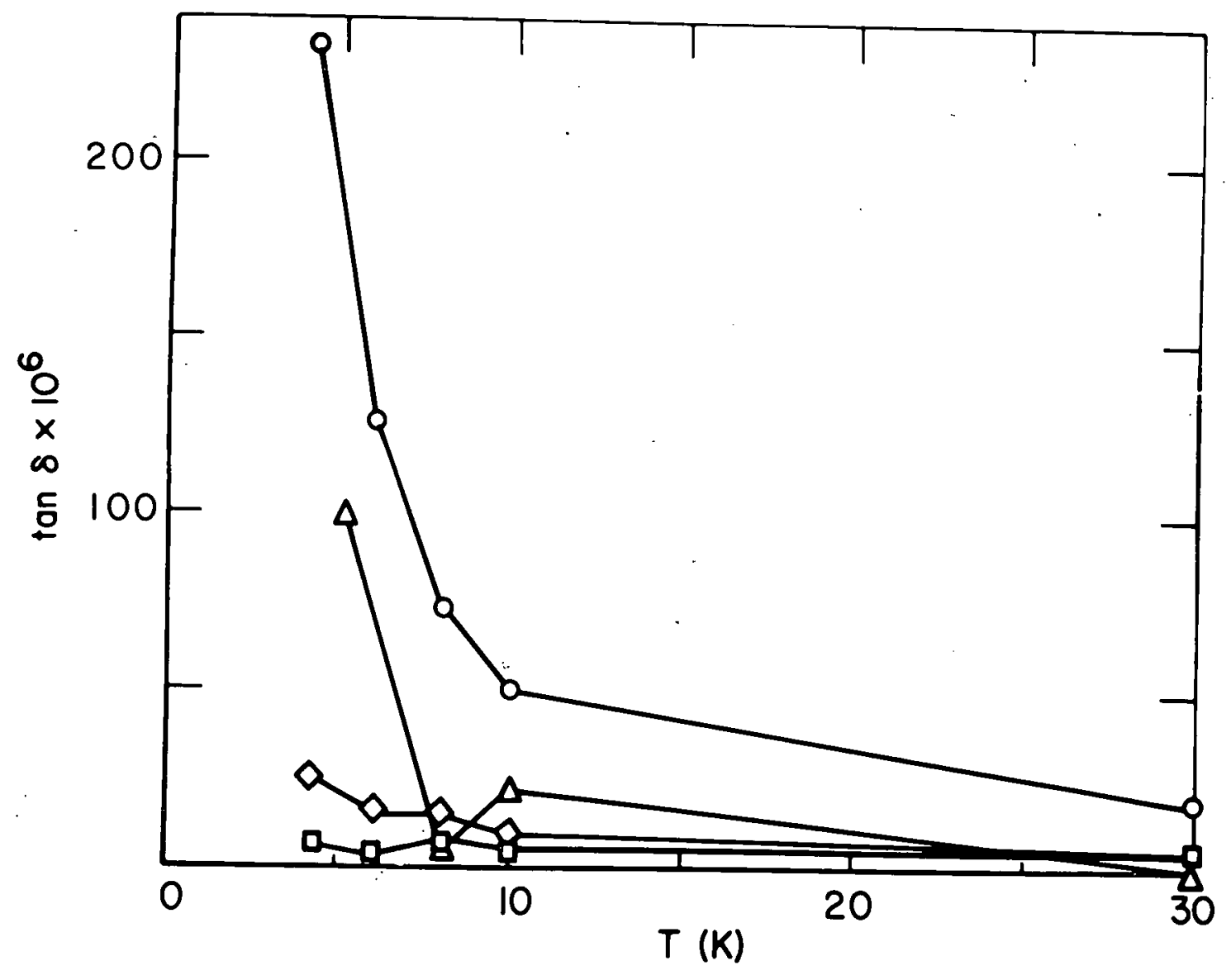

Figure 6: Tan $\delta$ vs. temperature at $100 \mathrm{~Hz}$ for various Polyethylene-BNL samples listed in Table III. Circles represent sample BNL-8, triangles, BNL-7, diamond, BNL-9, and squares, BNL-2. 
The Polyethylene-C film shows an additional loss peak compared with the Polyethylene-A at $260 \mathrm{~K}$ and generally higher loss levels above $125 \mathrm{~K}$, but below $125 \mathrm{~K}$, it seems indistinguishable from the Polyethylene-A. The Polyethylene-D film differs from the Polyethylene-A film even more. Above $125 \mathrm{~K}$ the loss is similar but larger than that of the Polyethylene-C. As the temperature decreases below $10 \mathrm{~K}$ the $100 \mathrm{~Hz}$ loss rises to a $\tan \delta$ of $12 \times 10^{-6}$ and the $1 \mathrm{kHz}$ loss to a $\tan \delta$ of $33 \times 10^{-6}$. This indicates that there is a definite loss peak in the Polyethylene-D below $4.2 \mathrm{~K}$ although the loss level in both the $\mathrm{C}$ and $\mathrm{D}$ films remains suitable at the power cable operating temperatures.

The other polyethylene samples measured consisted of 14 sheets (each about $200 \mu \mathrm{m}$ thick) prepared from the same linear polyethylene polymer by Battelle, for BNL. The sheets differed in that they had various amounts of antioxidant added and some were subsequently subjected to 24 hour extraction in different boiling solvents. The Polyethylene-BNL samples are listed in Table III. Sample BNL-2 was taken as characteristic of the base polymer for illustration in Figure 5. This film showed a very low loss level at all temperatures, no indication of increasing loss at room temperature, and a reduced peak at $150 \mathrm{~K}$. The low temperature data were similar to the Polyethylene-A data below $55 \mathrm{~K}$ except for a rise in tan $\delta$ at 1 $\mathrm{kHz}$ below $6 \mathrm{~K}$. In terms of dielectric properties, it too met all criteria.

The data for all the Polyethylene-BNL samples are given in Table V and representative data are illustrated in Figure 6. Because only single samples of each of the different sheets were measured and because their surfaces were not very flat, the loss data show additional scatter. Also, some of the samples had noticeable surface layers that were not easily removed with an acetone-ethanol-distilled water rinse which was given to all the samples. Some of the sheets had noticeable inclusions but they were avoided, when possible, in cutting the discs used in the cell and did not seem to effect the measurements. 
Samples BNL-1 and BNL-2 are made from the base polymer which.. contains only the antioxidant present in the original polymer, $0.015 \% \mathrm{WW}^{(1)}$. These two samples show the presence of a loss peak below $4 \mathrm{~K}$, with the unextracted BNL-1 having somewhat more loss. Both samples are very similar in behavior to previous samples of polyethylene. The other unextracted samples, which have antioxidants XX, YY, and $\mathrm{ZZ}$ added, all have enhanced low temperature loss with the peak still being below $4 \mathrm{~K}$. The loss, with one exception is proportional to the additive content and similar for all three additives. The exception is that the samples which had $0.1 \%$ antioxidant added seem to act as if they had only, $0.05 \%$ antioxidant. DSC data ${ }^{(1)}$ behaved in a like manner, so that this exceptional behavior of the $0.1 \%$ additive, content samples could be an artifact of the way the samples were prepared. The data on the BNL samples show that the presence of antioxidant in polyethylene can give rise to enhanced loss in polyethylene at cryogenic temperatures. The loss peak is below $4 \mathrm{~K}$ at the frequencies used here and is quite sharp with respect to frequency. It is not as sharp as a Debye loss peak, however, as can be seen by comparing the $100 \mathrm{~Hz}$ and $1 \mathrm{kHz}$ data. For a Debye loss peak tan $\delta$ would be proportional to frequency in the low frequency wing, whereas the data for sample BNL- 8 at $8 \mathrm{~K}$ show that the loss at $1 \mathrm{kHz}$ is larger than that at $100 \mathrm{~Hz}$ by a factor of 6 rather than 10 . Compared to normal polymer loss peaks, though, the loss peak.. below $4 \mathrm{~K}$ is still very sharp. Also, this enhanced loss is confined to low temperatures. Data on sample BNL-8, which shows the largest loss peak at cryogenic temperatures, were obtained over the entire temperature range. When the temperature was $80 \mathrm{~K}$ and above, the loss was very similar to that for sample BNL-2 which had the lowest loss at cryogenic temperatures of all the BNL samples. Thus at low frequencies, antioxidants seem to affect $\tan \delta$ only at cryogenic temperatures. 
Solvent extraction with methanol and cyclohexane does reduce the low temperature loss caused by the addition of antioxidant. For the one sample for which there is data, cyclohexane seems to reduce the loss to that of the original polymer while for the others with methanol the additional loss is reduced by about half. This loss reduction is seen even in the base polymer, thus sample BNL-2, which was extracted in methanol, has löwer loss than sample BNL-1, which was not. Since the original polymer contained antioxidant, this loss reduction is also related to the antioxidant content.

\subsubsection{Polypropylene}

Four different polypropylene films were measured. They are identified as Polypropylene-A, B, C, and D, respectively. The A and B samples were nominally the same except for surface treatment, the B having been corona treated. As the $A$ and $B$ films appeared identical, only the data for the A film will be mentioned. The sample thicknesses were $\mathrm{A}(27 \mu \mathrm{m}), \mathrm{C}(130 \mu \mathrm{m})$, and $\mathrm{D}(33 \mu \mathrm{m})$. The data are presented in Table VI and Figure 7.

Even more than in polyethylene, the loss data are quite different for the different polypropylene samples. This is true throughout the entire temperature range. The data for Polypropylene-C, a film with low crystallinity (see Section 2.2.2b) show the beginning of a loss peak above room temperature, a loss peak at $120 \mathrm{~K}$ and a decrease in $\tan \delta$ with decreasing temperature to about $10 \times 10^{-6}$ at $10 \mathrm{~K}$ and below. There is no evidence of a low temperature loss peak. 


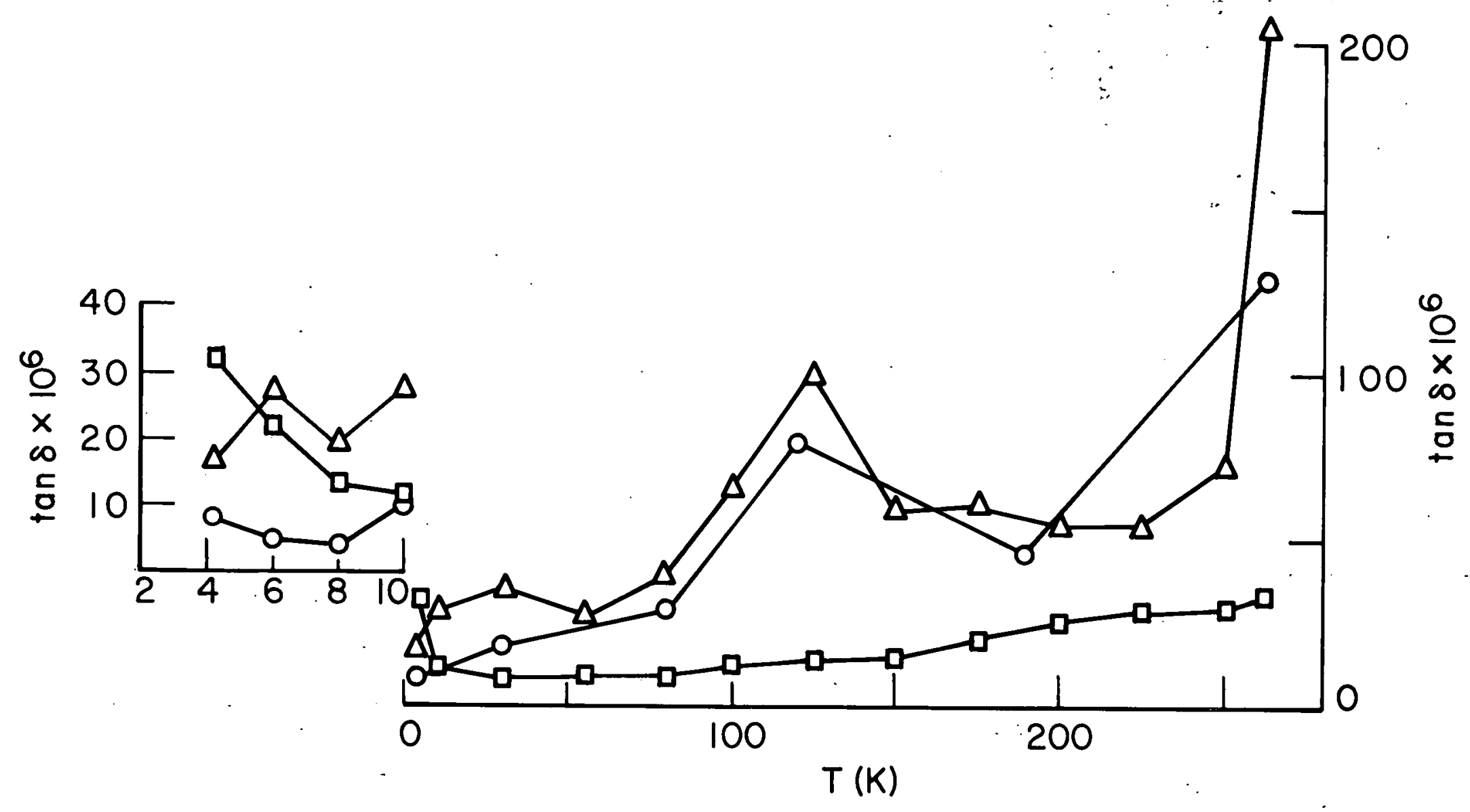

Figure 7: Tan $\delta$ vs. temperature at $100 \mathrm{~Hz}$ for various polypropylenes. The squares represent Polypropylene-A, the circles Polypropylene-C, and the triangles Polypropylene-D. 
Polypropylene-A shows just a gradual decline in tan $\delta$ from $30 \times 10^{-6}$ at room temperature to $9 \times 10^{-6}$ at $30 \mathrm{~K}$. The loss peak at $120 \mathrm{~K}$ exhibited by the Polypropylene-C seems to be completely supressed. As in the polyethylenes, there is a low temperature loss peak below $4 \mathrm{~K}$ and the loss rises to $32 \times 10^{-6}$ at $4.2 \mathrm{~K}$ at $100 \mathrm{~Hz}$. Thus, at $100 \mathrm{~Hz}$ the low temperature loss becomes equal to the room temperature 10ss, and it even exceeds it at $1 \mathrm{kHz}$.

The loss data for Polypropylene-D is different st111. The loss peaks found In the Polypropylene- $C$ are present except that the room temperature loss is somewhat less, and the $120 \mathrm{~K}$ peak a l1ttle more. In the $\mathrm{D}$ sample a new broad loss peak appears at $30 \mathrm{~K}$ that seems to persist down to $4 \mathrm{~K}$. There is no evidence of a loss peak below $4 \mathrm{~K}$, but tan $\delta$ stays between 20 to $30 \times 10^{-6}$ throughout the superconducting cable temperature range, making this polymer acceptable in terms of loss.

\subsection{Discussion}

The loss data for polycarbonate, polysulfone and Nylon 11 do not need much explanation. Only a few samples were measured for each and they are not currently favored candidates for the power cable insulation (2). The reasons range from poor mechanical properties in the case of Nylon 11 to poor electrical behavior for the others. Note that not many different forms of these polymers were available to show the kinds of differences that were found in polyethylene and polypropylene.

Polyethylene and polypropylene do warrant some extended discussion. Both polymers are considered to be low loss materials but the data show considerable differences in behavior throughout the temperature range. While many of the film samples seem to have very acceptable values of tan $\delta$ at cryogenic temperatures two samples approach the limit of acceptability and one of the two (Polypropylene-A) even has a higher loss at $4 \mathrm{~K}$ than at room temperature. 
All the polyethylene films (A, C, D) and the Polypropylene-A showed at least a hint of a loss peak below $4 \mathrm{~K}$. From the data on the Polyethylene-BNL samples, it is clear that the addition of antioxidants to polyethylene can give rise to such loss. This confirms, in a systematic manner, previous work ${ }^{(3)}$. Also the data suggest that the loss can get excessive at the cable operation temperatures. Furthermore, solvent extraction does reduce this source of loss. From the similarities in behavior, the appearance of a low temperature loss peak in all the polyethylene films and the Polypropylene-A can be assumed to be due to the presence of antioxidants.

Questions still remain with respect to antioxidants without even worrying about possible mechanisms. It is not as yet known whether there is any interaction between the polymer structure and the antioxidant content which affects the magnitude of the loss. It should be noted in this regard that when more highly oriented films having better mechanical properties become available, the possibility should be examined of whether they exhibit enhanced antioxidant induced loss. The present data are not able to answer this question.

The ability of solvent extraction to reduce the loss attributed to antioxidant is also a little puzzling in one respect. The use of chromatography seems to indicate that no more than $30 \%$ of the antioxidant was ever removed by extraction (1). This is not enough to account for the reduction in loss seen here or the change in DSC data (1). At tempts to monitor the antioxidant concentration by infrared and Raman spectroscopy at NBS were unsuccessful. If the chromatography results are correct, it is suggestive that the antioxidant effects are surface related. The current measurements are unable to answer this question. 
For Polypropylene-D, the loss below $10 \mathrm{~K}$ does not seem to be related to the presence of antioxidants. There is no indication of a peak below $4 \mathrm{~K}$ as seen in other samples. Rather $\tan \delta$ is almost constant between 4.2 to $10 \mathrm{~K}$. Also, the level of loss was higher between $6-8 \mathrm{~K}$ than any other polyethylene or polypropylene. If the possible antioxidant contribution is ignored, the loss below $10 \mathrm{~K}$ seems to be connected with a broad, weak loss peak near $30 \mathrm{~K}$. This peak has been seen before in mechanical measurements ${ }^{(4)}$ but its origin is unknown. It is possible that this loss peak is related to the structure of this film, (see Section 2.2.2c), but further work will have to be done to verify this. The results for Polypropylene-D do suggest that there are sources of loss at cryogenic temperatures that are not due to antioxidant level and could be troublesome.

\subsection{Summary}

The measurements of $\varepsilon$ and $\tan \delta$ on the polymer films looked at show that it is possible to meet the requirements for the insulation in superconducting power cable with polyethylene, polypropylene, and Nylon 11 . However, the measurements also show that it is possible to get excessive loss in these materials. Any modifications made to these polymers to enhance their mechanical properties will have to be examined for the possibility that they might affect their dielectric properties adversely. 
2. Aspects of the Fine Structures of Polymer Tapes (F. Khoury, J.P. Colson, L.H. Bolz)

\subsection{Introduction}

The purpose of the work described in this section was to establish some background knowledge of aspects of the fine structure of the various polymer tapes whose. dielectric and mechanical properties are being investigated as part of the ongoing screening process aimed at selecting tapes which are suitable for use as insulation in $\mathrm{Nb}_{3} \mathrm{Sn}$ superconducting cables.

Tapes which are slit from commercially available polypropylene, Nylon 1l, polycarbonate and polyethylene films are at present being evaluated for use in the cables. One aspect of the evaluation process is to compare the dielectric and mechanical properties of different tapes made from each of these polymers, the aim being to find whether any tape made from one of these polymers exhibits optimum dielectric and mechanical characteristics. The reasons for undertaking a concurrent examinatio. of the fine structure of the tapes are outlined below.

The structure of polymer films can vary widely with respect to the overall crystallinity of the film, the orientation of the crystallites in the film, the manifestation of chain-folding in the crystallites, and the occurence and tightness of tie molecules between neighboring crystallites. These various features are governed by the conditions under which the polymer crystallizes and the extent to which it is subjected, as is usual in film manufacture, to orientation inducing processes such as uniaxial or biaxial stretching or rolling ${ }^{(5)}$. Furthermore, the dielectric loss characteristics and, more particularly, the mechanical properties of polymers are sensitively structure dependent ${ }^{(6)}$. Thus, dielectric and mechanical losses can vary depending on the crystallinity of the polymer sample, and the tensile modulus 
of polymer samples in which the crystalline regions are unoriented can be as much as two orders of magnitude smaller $\left(x 10^{-2}\right)$ than that in a sample in which the crystalline regions are highly oriented with the chain molecules parallel to the straining direction. Accordingly, knowledge of the respective structures of different tapes made from the same polymer provides a basis for analyzing their physical properties, for assessing the possible origins of differences between their dielectric and/or mechanical properties, and for establishing guidelines on how the fine structure of the tapes could be controlled at the film manufacturing stage so as to optimize these properties. It should be noted in this connection that the physical properties of the tapes are not only of importance with regard to the functions of the tapes at cryogenic temperatures. The success of the polymer tape winding operation in the manufacture of the cables is critically dependent on the mechanical properties of the tapes at room temperature.

The main objective of our work at this exploratory stage has been to examine the nature of the orientations of the crystallites (crystalline orientation) in the tapes and to characterize, qualitatively, salient differences in orientation among the tapes. To this end, wide angle $x$-ray differaction (WAXD) has been used as a probe which, as will be seen, has also served to identify tapes having relatively poor crystallinities. In addition, small angle $x$-ray diffraction (SAXD) data have been obtained which pertain to the manifestation of chain-folding in the crystallites and which also have a bearing on the crystalline orientation in the tapes.

Apart from their dielectric and mechanical properties, there are other aspects of the polymer tapes which are being investigated at BNL and other laboratories in connection with either the cable manufacturing process (the tape winding operation) or the performance of the cables in end use. Two of these aspects are the frictional properties of the tapes, and the radial thermal conductivity at cryogenic temperatures 
of the helium bathed ( 0.5-1") thick layer of polymer tapes wound spirally around the core of the superconducting cable. A feature of interest in connection with both of these aspects is the nature of the interface between successive layers of tape. Some observations on the surface textures of a few of the polymer tapes are summarized in the last section of this report.

All of the tapes whlch have been studied were supplied by BNL.

\subsection{Crystalline Orfentation and Order in the Tapes}

\subsubsection{Background and Experimental}

Wide angle $x$-ray diffraction (WAXD see Glossary) patterns were recorded using a flat f1lm camera and $N 1-f 11$ tered CuKa $(\lambda=1.5418 \AA)$ radiation. Diffraction patterns were obtained with the $x$-ray beam orlented parallel to three different directions in each tape. These directlons which are 1dentified in Fig. 8, are the machine direction $M$ (1.e., the direction parallel to the long axis of the tape), the transverse or edge-on direction $T$ which is a right angle to $M$ and parallel to the plane of the tape, and $\mathrm{N}$ which 1 serpendicular to the plane of the tape. From a comparison of the wide angle $x$-ray diffraction patterns obtained in these three directions, deductions were made concerning the main characteristics of the crystalline orientation in the tapes, 1.e. the preferred orientation(s) of the constituent crystallites in the tapes.

In addition to the wide angle $x$-ray diffraction experiments, small angle $x$-ray diffraction (SAXD see Glossary) patterns of some of the tapes were recorded (on photographic film) using a small angle $x$-ray camera with pin-hole collimation and Ni-filtered CuK $\alpha$ radiation. These SAXD patterns were examined for the presence of discrete diffraction rings, arcs, or spots corresponding to spacings in the range 50-400A, the latter dimension being near the limit of resolution of the instrument. 


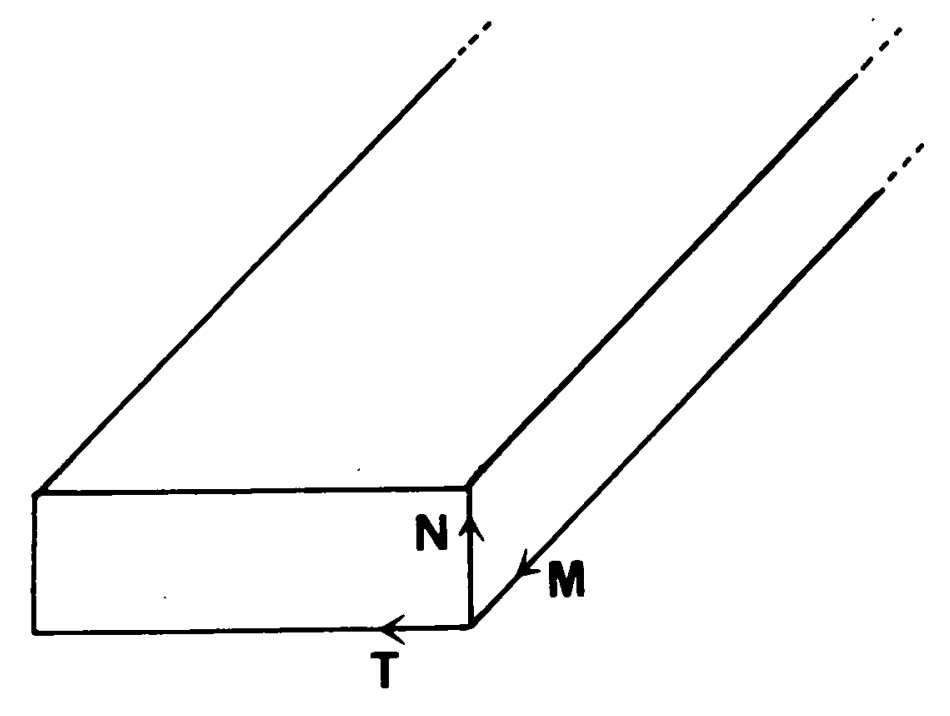

Figure 8: Identification of the axes of reference in the tapes. M is parallel to the machine direction which corresponds to the long axis of the tape. T is the transverse direction which is at right angles to $M$ and parallel to the plane of the tape. $N$ is normal to the plane of the tape. 
Briefly, the manifestation of such features is generally taken as evidence for the occurrence of chain folding in the crystallites. Furthermore, since in the simplest cases the chain molecules and hence the c-axis tend to be oriented at right angles to the fold surfaces of the crystallites information concerning the crystalline orientation in the tapes can also be derived from SAXD patterns. This latter type of information complements that obtained from WAXD patterns.

The density of some of the polyolefin tapes was measured using a water/ethyl alcohol density gradient column kept at $296 \mathrm{~K}$. These measurements remain to be checked for reproducibility; we nevertheless include them subject to duplicate determinations.

\subsubsection{Polypropy lene Tapes (Polypropylene-A, B. C, D, E)}

The most common unit cell structure of isotactic (see Glossary) polypropylene is monoclinic (see Glossary). The unit cell parameters are $a=6.65 \AA, b=20.96 \AA$, $c=6.5 \AA, b=99^{\circ} 20^{\circ},(7)$ and this cell is usually referred to as the $\alpha$-monoclinic ce11. Four chain molecules pass through the cell. The axis of each of these helical molecules is oriented parallel to the c-axis of the unit cell.

As an introduction to the crystalline orientations exhibited in the tapes the following characteristics of the manifestation of orientation in stretched polypropylene samples may be pointed out.

In common with other crystalline polymers, stretching polypropylene along one particular direction (uniaxial stretching) causes the crystallites to be oriented with their c-axis (which is parallel to the chain molecules) preferentially parallel to the stretching direction, the orientation of the crystallites is otherwise randomized in a cylindrically symmetrical manner about that direction. We shall refer 
to this type of orientation as c-axis orientation. Fig. 9a represents the WAXD pattern which would be obtained with the $\mathrm{x}$-ray beam oriented at right angles to the stretching direction in an ldealized sample having perfect c-axis orlentation parallel to the stretching direction. The calculated ${ }^{(8)}$ dispositions of the diffraction spots corresponding to the (110), (040), (130), (111), (13I) and (041) crystal planes are Indicated in the diagram as they would be recorded on a flat photographic film. A pecullarity of unfaxially stretched polypropylene should be noted, however. Thus, in addition to the manifestation of c-axis.orlentation parallel to the stretching direction and depending on the extent of stretching, on the thermal conditions under which stretching takes place; and on thermal aftertreatments, a varylng but usually relatively minor proportion of the crystallites In unlaxially stretched samples are orlented with their a-axis (or possibly the $a^{\prime}$-axis, where $a^{\prime}$ is the projection of the a-axis onto the plane perpendicular to the c-axis) parallel to the stretching direction $(9,10)$. The orientation of this latter population of crystallites is otherwise randomized in a cylindrically symmetrical fashion about the stretching direction. We shall refer to this type of orientation as a-axis orlentation, and Fig. $9 \mathrm{~b}$ represents the corresponding calculated WAXD pattern.

The simultaneous occurence of two populations of crystallites, one with c-axis orientation and the other with a-axis orientation, both parallel to the stretching direction in uniaxially stretched polypropylene is believed to be a consequence of, or related to, a mode of twinned crystal growth which is unique to polypropylene. The main feature of this twinning phenomenon is that the c-axis in one twin is parallel to the a-axis in its companion, the b-axis in each twin being parallel to that in its companion (11). We shall not attempt to discuss current views on the 


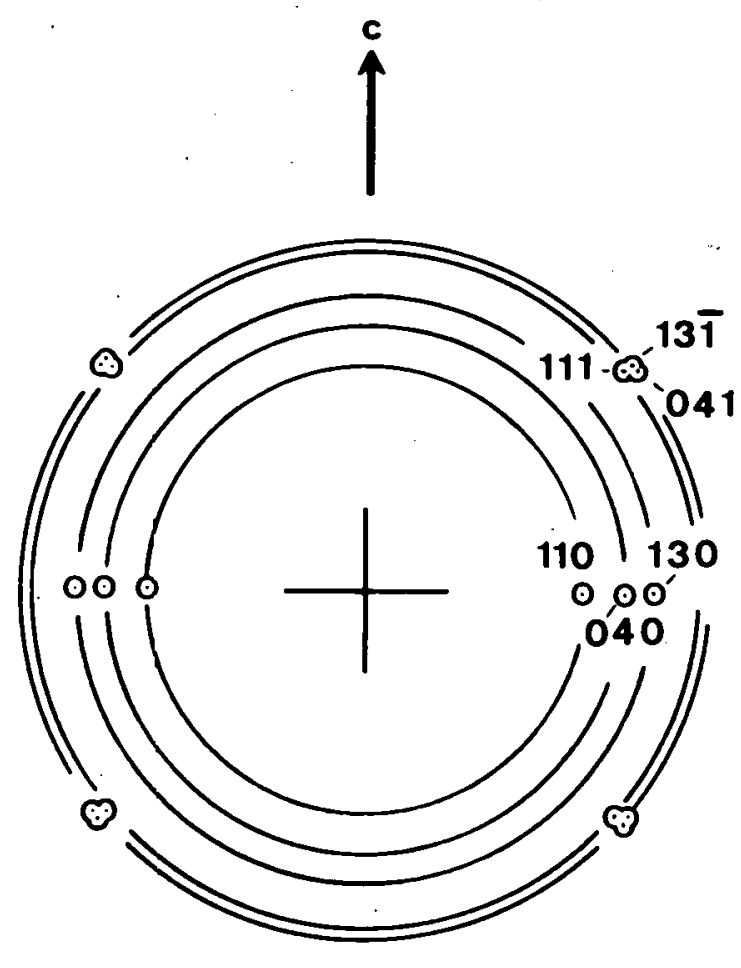

(a)

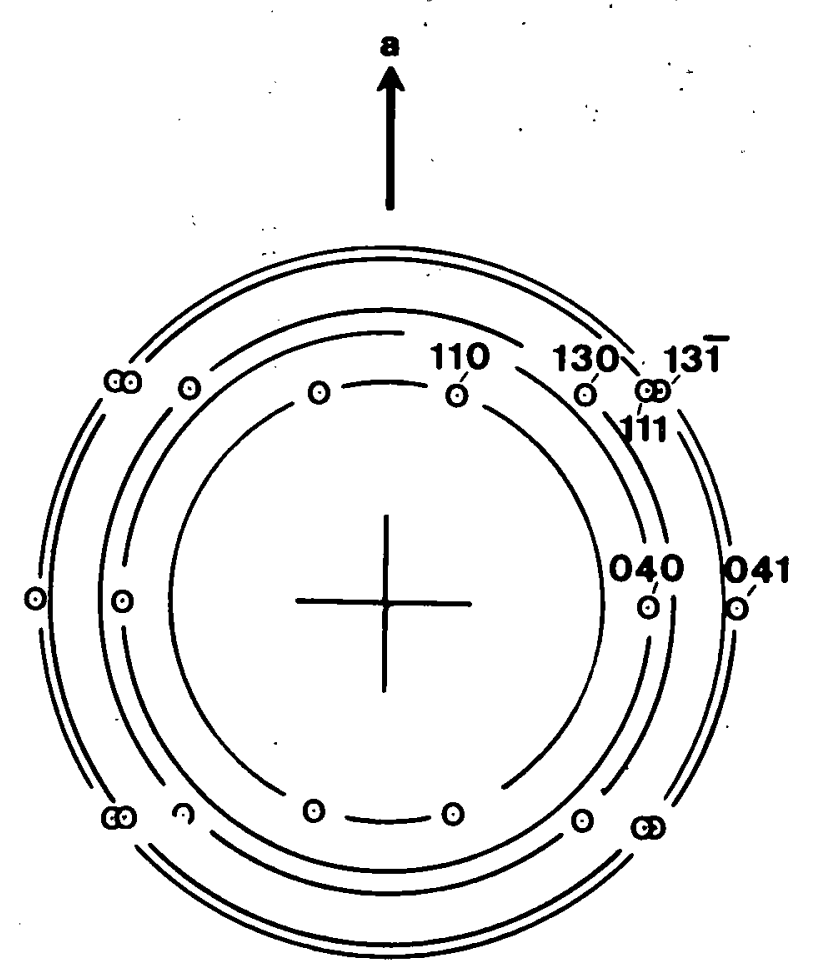

(b)

Figure 9: Calculated dispositions of 110, 040,130,111, 13ī, and 041 reflections in:

(a) A WAXD pattern of a polypropylene sample having c-axis orientation (see text). The crystallites are oriented with their c-axis parallel to the vertical direction in the diagram. The $x$-ray beam is normal to the plane of the figure.

(b) A WAXD pattern of a polypropylene sample having a-axis orientation (see text). The crystallites are oriented with their a-axis parallel to the vertical direction in the diagram. The $x$-ray beam is normal to the plane of the figure: 
origins of this phenomenon ${ }^{(11-13)}$. It is sufficient in the present context to note that composite WAXD patterns corresponding to a parallel superposition of the two patterns depicted in Fig. 9 are often exhibited by uniaxial stretched polypropylene samples. In actuality, of course, diffraction arcs rather than sharp spots are observed in experimental WAXD patterns due to deviations among the crystallites from the idealized orientations represented in Fig. 9.

More intricate orientations or mixtures of orientations than occur in uniaxially stretched samples are manifested in samples subjected to more complicated stretching regimes, e.g. simultaneous or stepped biaxial stretching (balanced or unbalanced), ${ }^{(14-17)}$ cold rolling, ${ }^{(18)}$ or uniaxial compression (19).

Confining our remarks to the case of biaxial stretching, it is sufficient for our present purpose to indicate, with reference to Figs. 10a and 10b, two preferred types of crystallite orientation which have been reported to occur simultaneously (and in some cases in combination with both c-axis and a-axis orientations) in biaxially stretched films (14-17).

Adopting a nomenclature used by Adams, ${ }^{(17)}$ the orientation illustrated in Fig. 10a will be referred to as (040) planar orientation. This designation identifies a population of crystallites in which the (040) planes are parallel to the plane (MT) of the film. The b-axis in these crystallites is parallel to $N$, their orientations are otherwise randomized in a cylindrically symmetrical fashion about N. It follows that the c-axis in the crystallites is parallel to the plane (MT) of the film, but there is no net preferred orientation of the c-axis along any direction in that plane. Fig. 10a represents the calculated dispositons of the 110, 040, 130, $111,13 \vec{I}$, and 041 diffraction spots in a WAXD pattern taken with the $x$-ray beam parallel to either $\mathrm{T}$ or $\mathrm{M}$ (or any direction parallel to the MT plane) in a film exhibiting (040) planar orientation. 


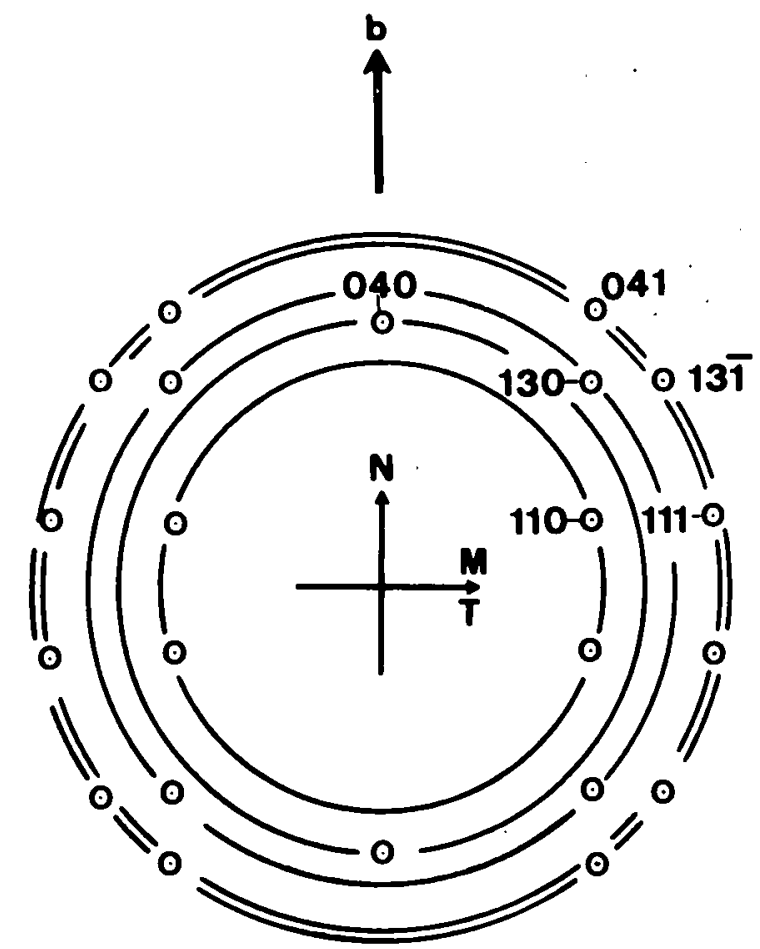

(a)

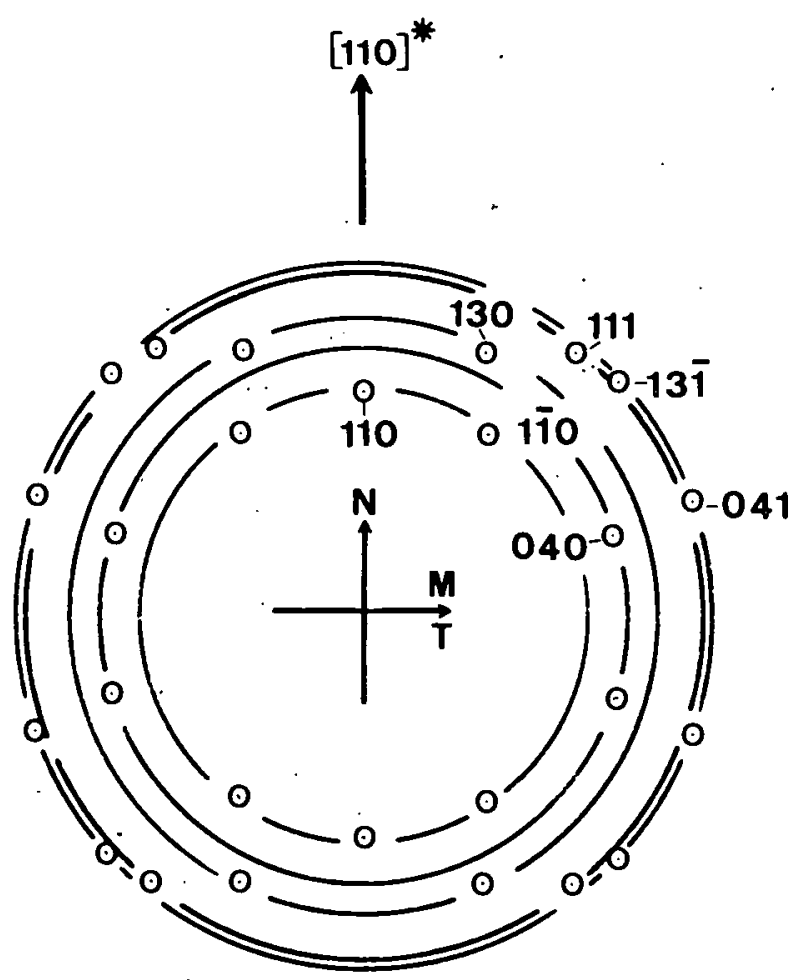

(b)

Figure 10: Calculated dispositions of the $110,1 \overline{1} 0,040,130,111,13 \overline{1}$, and 041 reflections in WAXD patterns taken with the $x$-ray beam along $M, T$, or any direction parallel to the MT plane in films having the following respective orientations: (a) (040) planar orientation, the (040) planes in the crystallites are oriented parallel to the MT plane of the film and the b-axis is normal to the MT plane (see text for more details). (b) (110) planar orientation, the $\{110\}$ planes are oriented parallel to the MT plane of the film and the $[110]^{*}$ or [110]* reciprocal lattice axes are normal to the MT plane (see text). 
The orientation illustrated in Fig. 10b, referred to as $\{110\}$ planar orientation, (17) Identifies a population of crystallites in which $\{110\}$ planes i.e., the (110) or the (1 10$)$ planes) are parallel to the plane (MT) of the film. Correspondingly, the $[110] *$ or the $[1 \overline{10}] *$ reciprocal lattice axes in the crystallites are parallel to $\mathrm{N}$ and the orientations of the crystallites are otherwise randomized in a cylindrically symmetrical fashion about $N$. In addition the c-axis in the crystallites is oriented parallel to the (MT) plane of the film. Fig. 10b represents the calculated dispositions of the $110,1 \overline{1} 0,040,130,111,13 \overline{1}$, and 041 diffraction spots in a WAXD pattern taken with the $x$-ray beam paralle1 to either $T$ or $M$ (or any direction parallel to the MT plane) in a film exhibiting $\{110\}$ planar orientation.

M-axis or T-axis WAXD patterns obtained from a film in which both the (040) planar and the $\{110\}$ planar orientations are manifested, would correspond to a parallel superposition of the calculated patterns shown in Fig. 10a and Fig. 10b. In actuality, due to deviations from the perfect orientations depicted in Fig. 10 the reflections will be arced. The orientations depicted in Fig. 10 represent in fact the preferred orientations of the crystallites and correspond to peaks in the distribution of crystallite orientations in the films. There is another feature of the diagram depicted in Figs. $10 \mathrm{a}$ and $10 \mathrm{~b}$ which should be pointed out. In the case of perfect (040) planar orientation, the (040) planes which are oriented parallel to the plane of the film will not satisfy the Bragg conditions for diffraction when the $x$-ray beam is parallel to $M$ or $T$, so that the $(040)$ reflections which are situated along $\mathrm{N}$ in Fig. 10a should have been omitted in principle.' The same consideration applies for the 110 reflections situated along $N$ in the case of $\{110\}$ planar orientation depicted in Fig. 10b. Due to the distribution in the 
rientations of the $(040)$ and $(110)$ planes in the crystallites relative to the ideal orientations represented in Figs. $10 \mathrm{a}$ and $10 \mathrm{~b}$ respectively, one would expect M-axis or T-axis diffraction patterns from films to exhibit in practice 040 reflections along. N. corresponding to preferred (040) planar orientation, and 110 reflectlons also along $\mathrm{N}$, corresponding to preferred $\{110\}$ planar ortentation. We have therefore marked the dispositions of these reflections in the respective diagrams since they are to be expected, and are Indeed observed diagnostic features (14-17).

\subsection{2a Polypropylene-A and Polypropy lene-B}

Since the WAXD patterns exhlbited by the Polypropylene-A tape were similar to those obtalned from the Polypropylene-B, we 11lustrate in F1g. 11 examples from the former tape only. The patterns may be indexed according to the $\alpha$-monoclinlc untt cell of polypropylene. The following features may be noted in F1g. 11:

(1) The M-axis WAXD pattern (1.e., diffraction pattern with the $x$-ray beam parallel to M In Fig. 1la, and the T-axis WAXD pattern in F1g. Ilb, in both of which the reflections are distinctly arced, are essentailly indistinguishable from one another. This feature taken together with the ringed character of the N-axis WAXD pattern, Fig. 1Ic, strongly suggests the existence of a crystalline orientation, or orientations, which are cylindrically symmetrical about $\mathrm{N}$.

(1i) Intense 110 and 040 arcs occur along $N$ in both Fig. 1la and Fig. 11b. From a consideration of Fig. 10, it can be seen that these two features are consistent with the occurence of preferential (110 planar orientation and preferential 040 planar orientation respectively in the tape.

(iii) Considering the case of 040 planar orientation first, it can be seen from Fig. 10a that one would expect the occurence of 110 arcs situated along two diameters oriented at close to $\pm 72.5^{\circ}$ relative to $\mathrm{N}$ in both $\mathrm{M}$ and $\mathrm{T}$ axis WAXD patterns. Such ares are present in Figs. 11a and 11b. Examination of Fig. 10a also shows that one 
a)
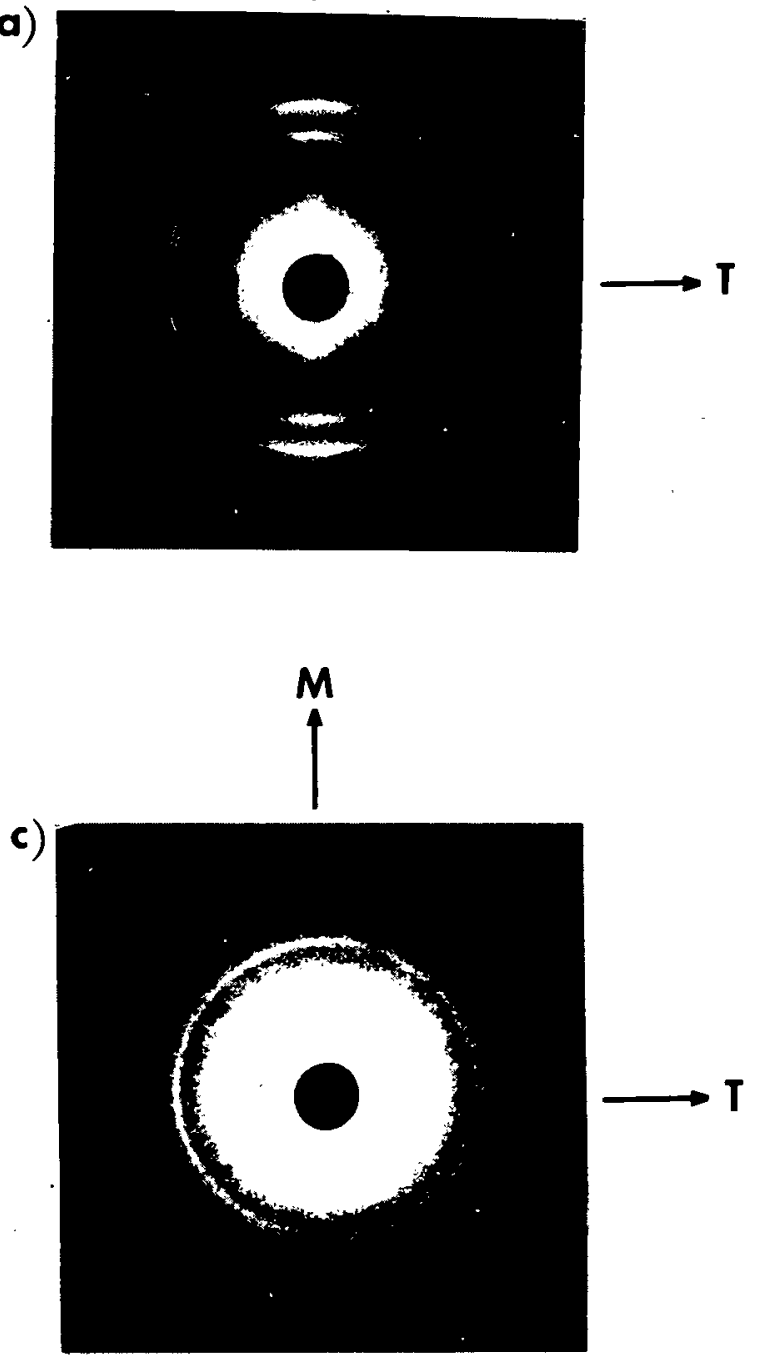

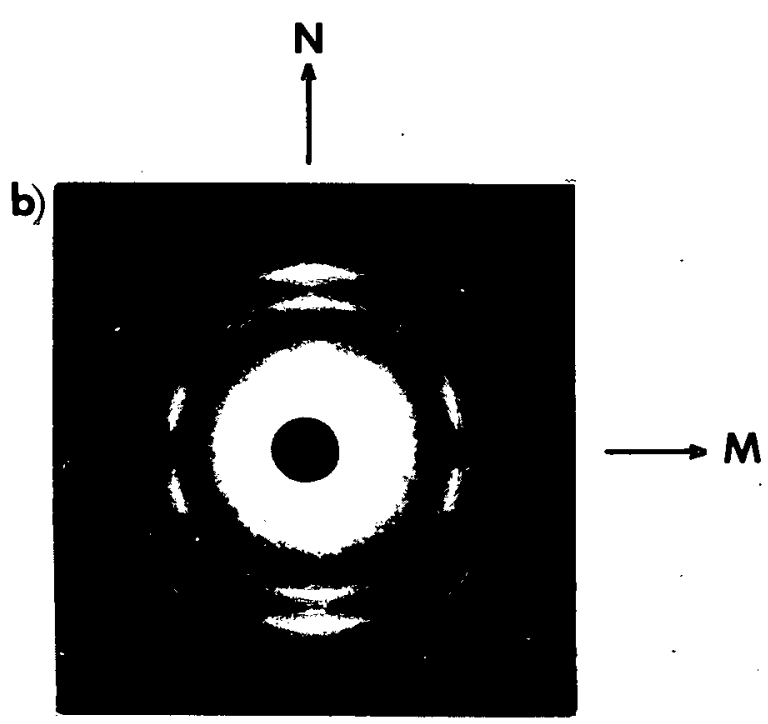

Figure 11: (a) M-axis, (b) T-axis, (c) N-axis, WAXD patterns from the Polypropylene-A tape. The specimen to film distances were not identical. These are contact prints of the originals. The two intense arcs situated along $N$ in (a) and (b) are 110 and 040 reflections respectively. The innermost ring in (c) is the 110 ring: 
would expect 111 arcs to occur along two diameters oriented at close to $\pm 78^{\circ}$ relative to N. Although faint, such arcs can be discerned in both Figs. 1la and $11 \mathrm{~b}$ - they were clearly evident in the original films. In short, the features indicated above are consistent with and indeed confirm the occurrence in the tape of a population of crystallites having preferential (040) planar orientation.

(iv) Turning to the case of (110) planar orientation, it can be seen from Fig. $10 \mathrm{~b}$ that one would expect 040 arcs to occur along two diameters oriented at close to $\pm 72.5^{\circ}$ relative to $\mathrm{N}$ in both $\mathrm{T}$-axis and $\mathrm{M}$-axis WAXD patterns. Such arcs are present in Figs. 11a, and 11b. A consideration of Fig. 10b also indicates that one would expect the occurences of 110 arcs along diameters oriented at $\pm 34^{\circ}$ relative to $N$. No such discrete arcs can be convincingly resolved in Figs. 1la and 11b. This may be attributed to the merging of these arcs with the 110 arcs situated along $N$, and the 110 arcs situated along diameters at $\pm 72.5^{\circ}$ to $\mathrm{N}$ which were identified above as, resulting from (040) plane orientation. Such merging would account for the apparent continuity between these last two sets of arcs. This "consideration and the clearer evidence provided by the occurrence of the 110 arcs along $\mathrm{N}$ and 040 arcs along diameters at $\pm 72.5^{\circ}$ to $\mathrm{N}$ certainly point to the occurrence in the tape of a population of crystallites having preferentail $\{110\}$ planar orientation.

We shall not attempt to analyze and discuss the diffraction patterns further, except to point out that a consideration of the patterns in Figs. 11a and 11b in the light of the calculated patterns shown in Fig. 10 with reference to the dispositions of the (130), (13i) and (041) reflections arising from both $(040)$ and (110) planar orientations, confirms the deductions pointed out above, namely that two identifiable populations of crystallites, one with preferred 040 planar orientation and one with preferred $\{110\}$ planar orientation, occur in the Polypropylene-A and 
Polypropylene-B tapes. It must, however, be emphasized that these two orientations are preferred orientations and that, as evidenced by the arced character of the reflections and continuities between others due to apparent overlaps, appreciable deviations from the idealized cases represented in Fig. 10 occur among the crystallites.

A SAXD pattern was taken with the $\mathrm{x}$-ray beam parallel to $\mathrm{N}$ in the Polypropylene-A tape, no small angle reflections could be discerned in the photographic film. This may be due to a broad distribution of fold periods in the crystallites. Annealing the tapes at elevated temperatures and examination of the SAXD patterns exhibited by the annealed specimens would shed further light on this matter which is however not being pursued at this juncture.

Finally, the density of a sample of the Polypropylene-A tape and one from the Polypropylene-B tape were measured and found to be $0.904_{5}$ and $0.904_{2}$ respectively. These values correspond to a weight fraction crystallinity of 0.63 .

\section{$2.2 .2 b$ Polypropylene-C}

No detectable evidence of crystalline orientation was observed in the WAXD patterns obtained from this type of tape. In addition, the WAXD patterns were indicative of poor crystalline order. The patterns differed from the type of pattern expected for unoriented $\alpha$-monoclinic polypropylene. Fig. 12a represents a diffraction pattern obtained with the $\mathrm{x}$-ray beam parallel to $\mathrm{N}$. A diffraction pattern from the same tape after it had been annealed at $413 \mathrm{~K}$ for two hours is shown in Fig. 12b, the $\mathrm{x}$-ray beam was also paralle1 to $\mathrm{N}$ in this case. The powder (ringed) type pattern exhibited by the annealed sample is typical of $\alpha$-monoclinic polypropylene as evidenced by the five distinct rings which, proceeding from the inner ring outwards, can be identified as the 110 , the 040 , the 130 , the 111 and the composite $13 \bar{I}, 041$ diffraction rings. In contrast, the pattern from the unannealed (as received) tape shown in Fig. 12a exhibits what appears, at first examination, to be only two diffuse rings 
a)
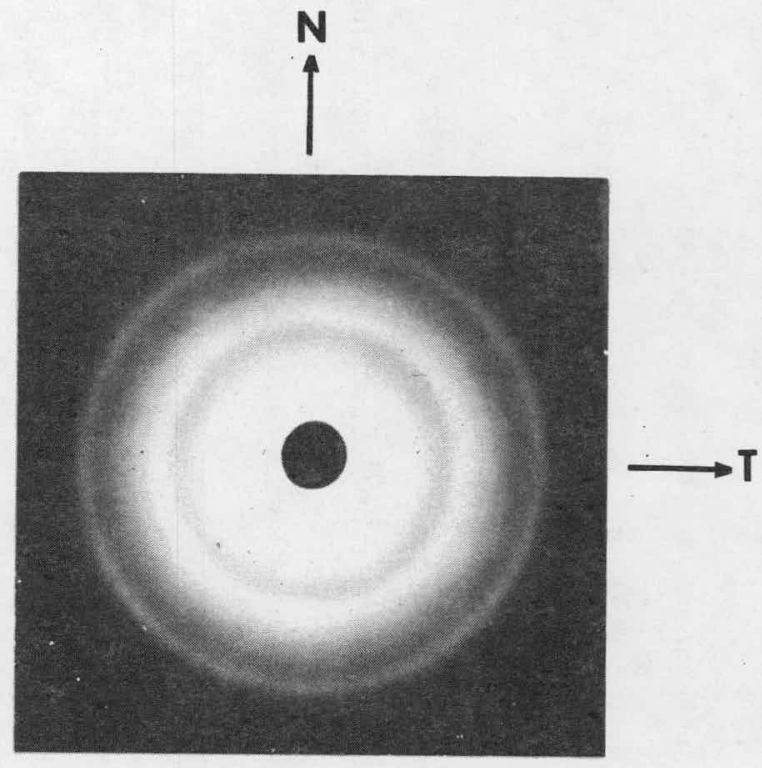

c)

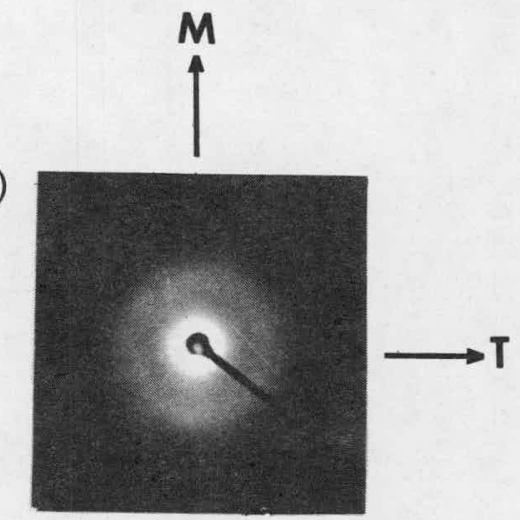

b)
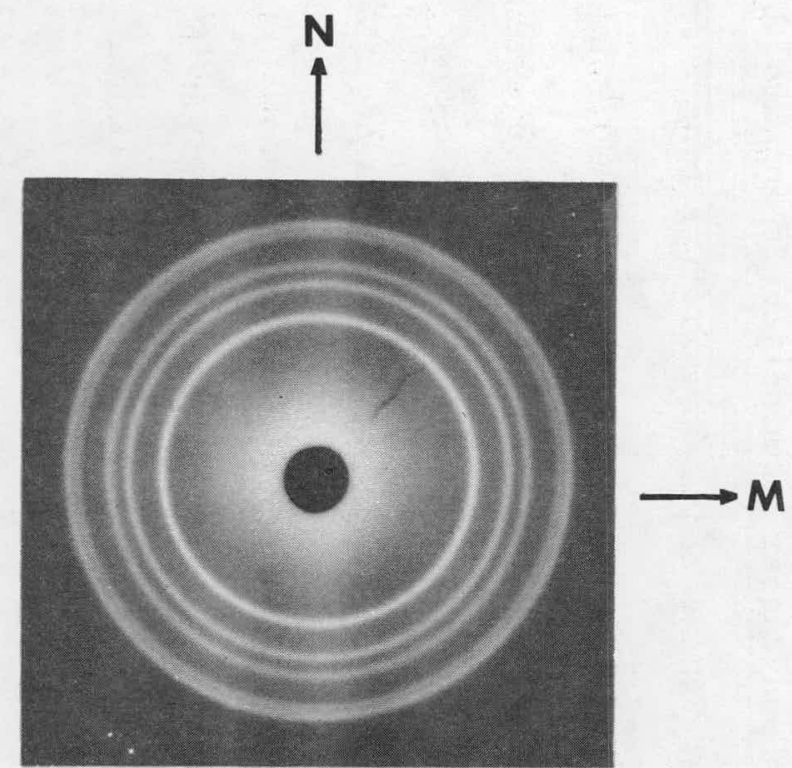

Figure 12: (a) N-axis WAXD pattern from the Polypropylene-C tape. (b) N-axis WAXD pattern from the same tape after annealing at $413 \mathrm{~K}$ for two hours. Specimen to film distances for (a) and (b) were not identical, these are contact prints of the originals, see text for comparison between the patterns.
(c) N-axis SAXD pattern of the unannealed tape. 
corresponding to spacings of $\sim 4 \AA$ and $\sim 6 \AA$. Upon closer examination it appears that the inner ring is a composite of a relatively sharp and a more diffuse ring. It may be concluded that the crystalline order in the tape, as received, lies between that of the smectic mesomorphous state of polypropylene ${ }^{(20)}$ (which state of order occurs when the polymer is rapidly cooled from the molten state) and the $\alpha$-monoclinic crystalline form associated with samples crystallized or annealed at relatively elevated temperatures.

The density of a sample of the unannealed tape was found to be $0.884_{3}$ which corresponds to a weight fraction crystallinity of 0.39 . This low crystallinity is consistent with the WAXD results described above.

A SAXD pattern obtained from the unannealed (as received) tape with the $x$-ray beam parallel to $\mathrm{N}$ is shown in $\mathrm{Fig} .12 \mathrm{c}$ in which a readily distinguishable diffraction ring can be seen. This ring corresponds to a spacing of $110 \AA$. The occurrence of such a diffraction ring is consistent with the presence of a chain-folded lamellar morphology and a random orientation of the lamellae in the tape. This latter feature is in turn consistent with the absence of evidence for crystalline orientation in the WAXD patterns.

\subsection{2c Polypropylene-D}

The M-axis, T-axis and N-axis WAXD patterns obtained from this tape are shown in Figs. 13a, 13b, and $13 \mathrm{c}$ respectively. These patterns can be indexed according to the $\alpha$-monoclinic unit cell of polypropylene. Conciseness and simplicity in description are better served in this case by first summarizing the conclusions concerning the orientation characteristics of this tape. The evidence, based on Fig. 13, will subsequently be described. 
a)
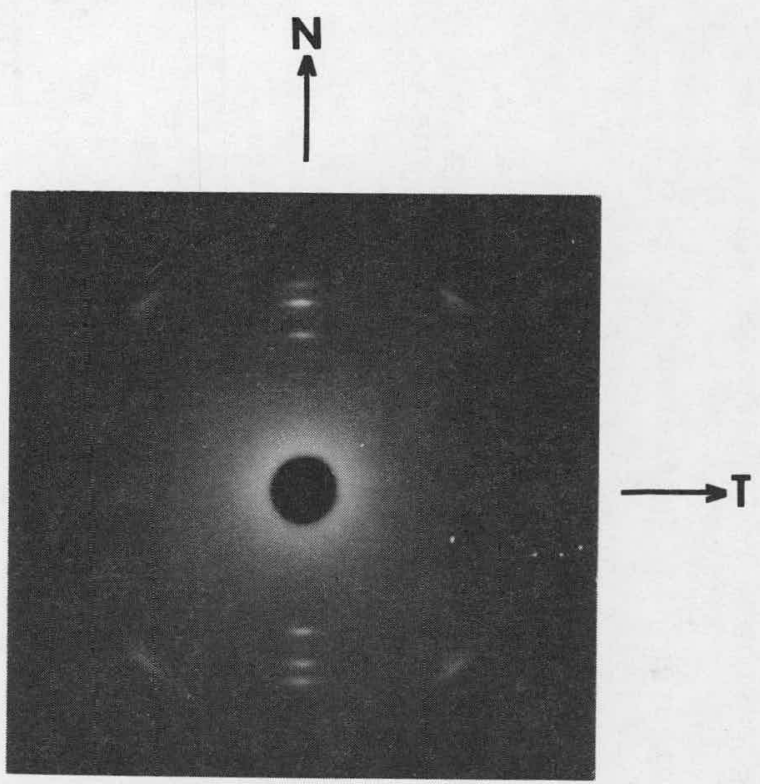

c)
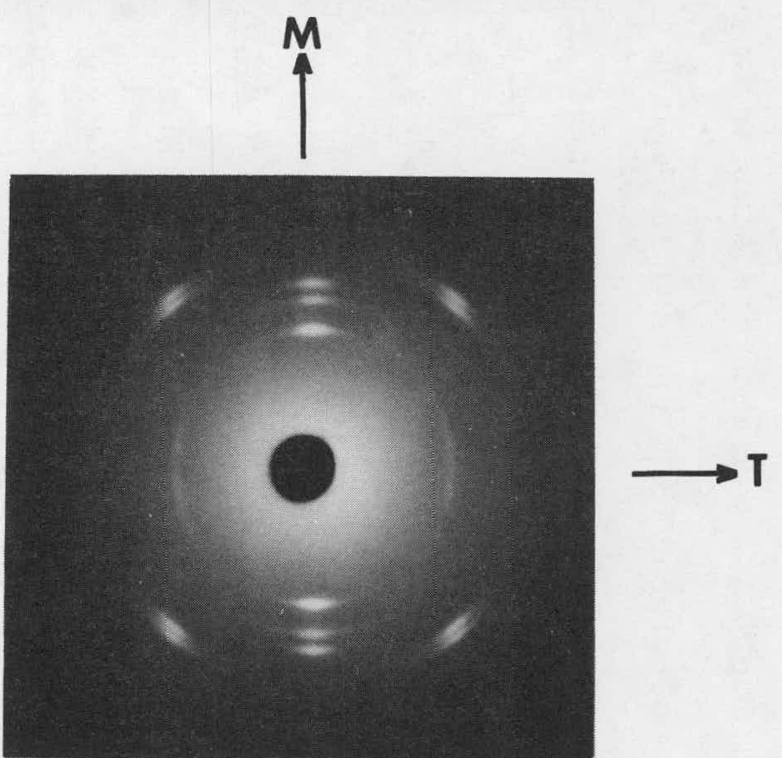

b )

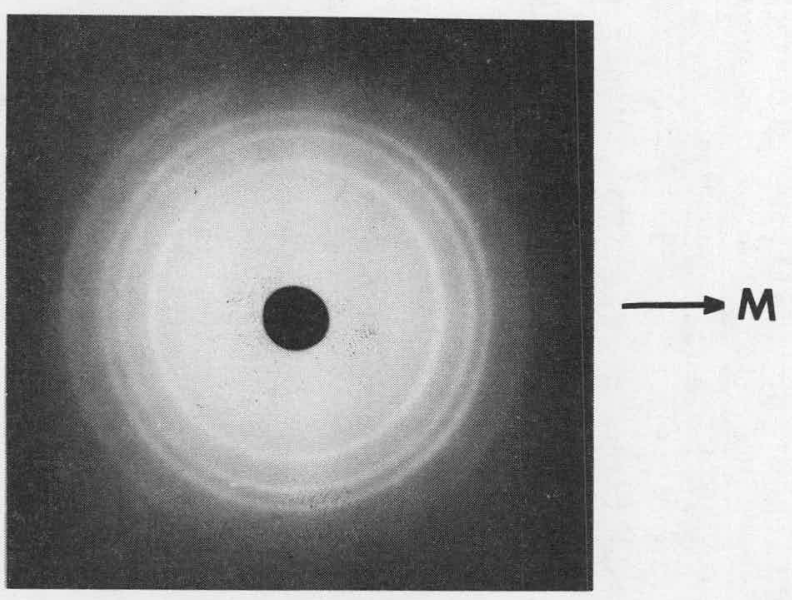

d)

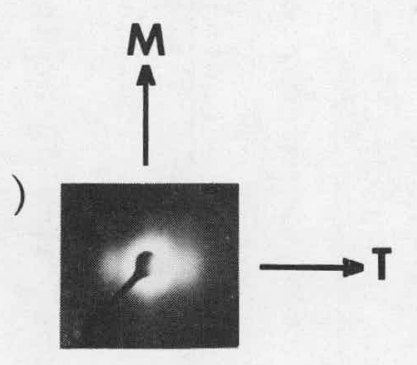

Figure 13: (a) M-axis, (b) T-axis, (c) N-axis WAXD patterns from the Polypropylene-D tape. The specimen to film distances are not identical, these are contact prints of the originals. The three intense arcs situated along $\mathrm{N}$ in (a) and $\mathrm{M}$ in (c) are the 110,040 and 130 reflections respectively. The three innermost rings in (b) are 110, 040 and 130 rings. (d) $N$-axis SAXD pattern from the same tape. 
The predominant feature of the crystalline orientation characteristics in this tape is the cylindrically symmetrical orientation of crystallites about the T-axis of the tape with the c-axis (and hence the chain molecules) in the crystallites oriented preferentially parallel to T. In addition, a relatively minor population of crystallites in which the a-axis in the crystallites is oriented preferentially parallel to $\mathrm{T}$, is also present in the tape. In short, the c-axis orientation and the a-axis orientation, which were mentioned in Section 2.2.2 (see also Fig. 9) as occuring simultaneously in certain circumstances in uniaxially stretched polypropylene, also occur in this tape. The important feature to note in the case of this tape, however, is that the c-axis and the a-axis orientations of the two populations of crystallites are respectively oriented parallel preferentially to the transverse direction $\mathrm{T}$ in the tape and not parallel to $\mathrm{M}$. This indicates that the film from which this tape was slit was stretched predominantly in the transverse direction $\mathrm{T}$.

The first aspect to note in Fig. 13 is that the M-axis (Fig. 13a) and the $\mathrm{N}$-axis (Fig. 13c) WAXD patterns are similar to one another. Furthermore, consideration of the calculated WAXD pattern in Fig. 9a indicates that preferential c-axis orientation (and hence preferential stretching) parallel to $\mathrm{T}$ in the tape would result in the occurrence of 110, 040, and 130 arcs situated along $N$ and $M$ in M-axis and $\mathrm{N}$-axis WAXD patterns respectively. This is indeed found to be the case in the corresponding patterns in Fig. 13a and 13c. Further, reference to Fig. 9a indicates that 111,041 and $13 \overline{1}$ arcs would be expected to occur along diameters at $\pm 40^{\circ}$ to the axis along which the 110,040 , and 130 arcs are situated. This is found to be so in both Fig. 13a and Fig. 13c, and confirms the occurrence among the crystallites of preferential c-axis orientation parallel to $\mathrm{T}$. 
The evidence for crystallites having preferential a-axis orientation parallel to $\mathrm{T}$ in this tape is by comparison very weak and limited to the occurrence of two faint and overlapping (110) arcs which straddle the T-axis in the M-axis and $\mathrm{N}$-axis diffraction patterns. Such arcs are faintly perceptible in Figs. 13a and 13c, they could, however, be discerned more clearly in the original x-ray films.

A small angle $x$-ray diffraction pattern taken with the $x$-ray beam parallel to $\mathrm{N}$ is shown in Fig. 13d. Two diffraction spots corresponding to a spacing of $280 \AA$ can be seen situated along $\mathrm{T}$. This pattern indicates in itself the occurrence in the tape of chain-folded crystallites which are oriented with their fold surfaces perpendicular to $\mathrm{T}$, and hence with the c-axis and the chain molecules parallel to $\mathrm{T}$, which features are consistent with the WAXD observations, and thus provide further confirmation that the predominant orientation among the crystallites in this tape is preferential c-axis orientation parallel to $\mathrm{T}$.

The density of a sample of this tape was found to be $0.908_{5}$ which corresponds to a weight fraction crystallinity of 0.69 .

\subsection{2d Polypropylene-E}

The M-axis, T-axis and N-axis WAXD patterns obtained from this tape are shown in Fig. 14. These patterns can be indexed according to the $\alpha$-monoclinic unit cell of polypropylene. An examination of these patterns leads to the conclusion that there apparently exist in this tape four distinguishable populations of crystallites having different preferred orientations namely, (040) planar orientation, (110) planar orientation, c-axis orientation parallel to $\mathrm{T}$, and a-axis orientation also parallel to $T$. In short, the two orientations identified in the Polypropylene-A tape and the two identified in the Polypropylene-D tape occur collectively in this tape. We shall not attempt to discuss the patterns or the complexities involved 
a )
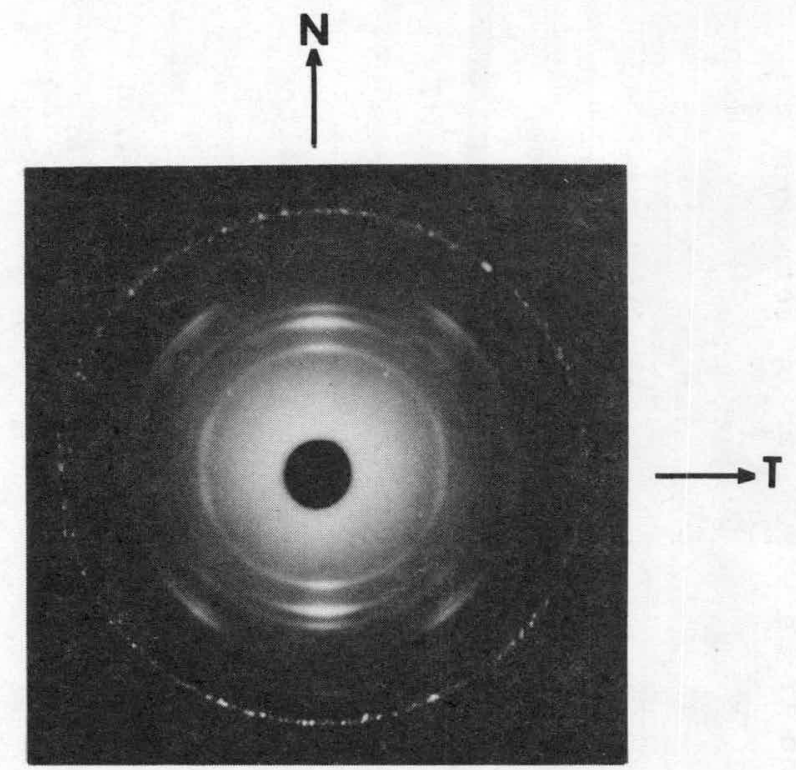

M

c)
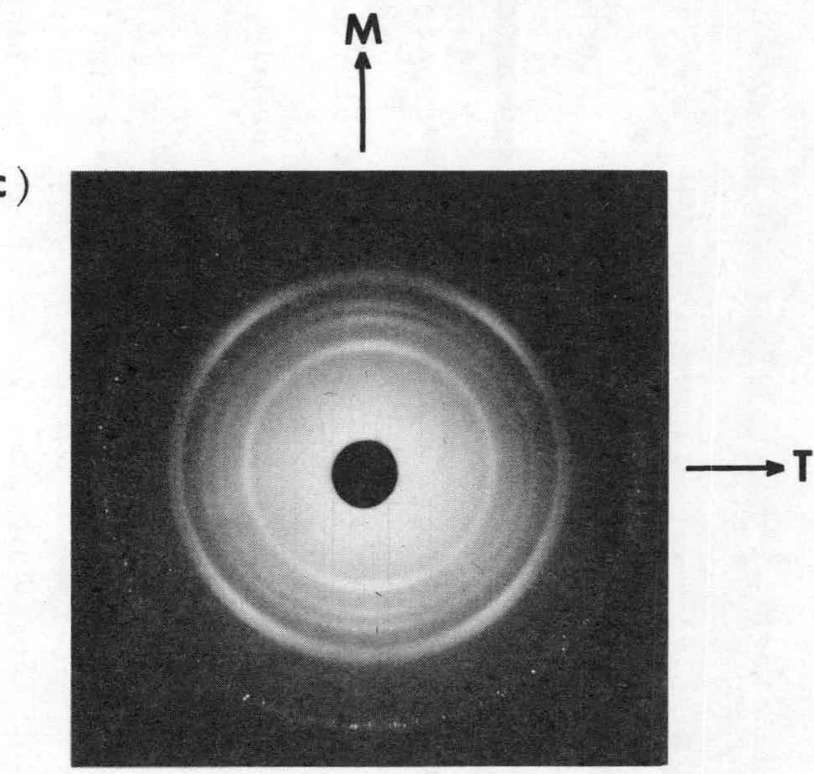

b)

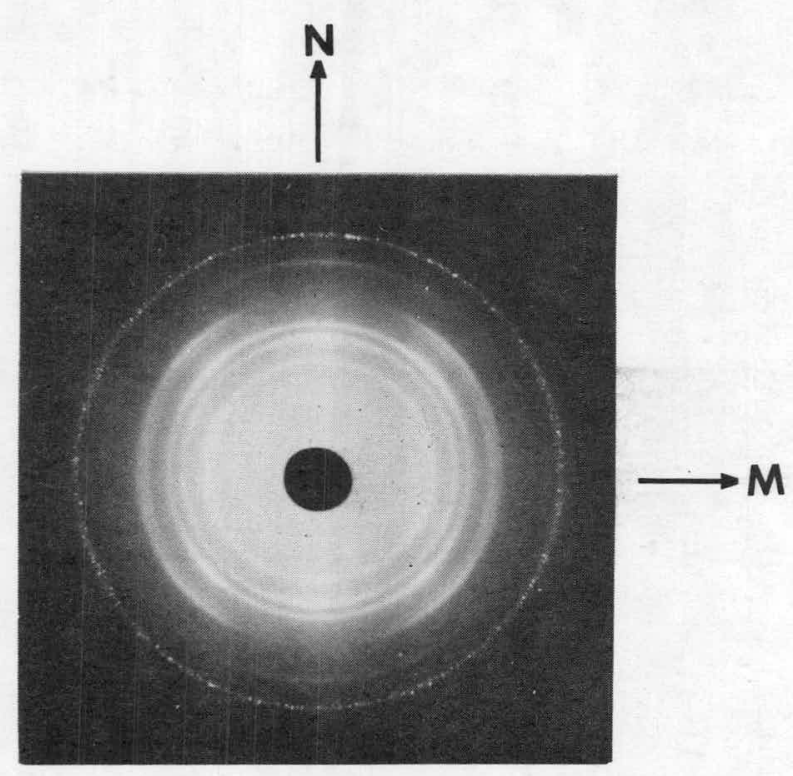

Figure 14: (a) M-axis, (b) T-axis, and (c) N-axis, WAXD patterns from the Polypropylene-E tape. The spotty outer ring in each pattern is the 111 ring from polycrystalline Si used for calibrating the specimen to film distance which is not the same in all three diagrams. 
in their analysis. We will limit ourselves to pointing out that the simultaneous existence of these four orientations in the same tape results in the occurrence, in the WAXD patterns, of several complicating coincidences and overlaps between arcs corresponding to particular crystallographic planes (e.g., (110) or (040)) in the crystallites of the differently oriented populations. These complications can be judged, in part for example, from a consideration of the superposition of the calculated patterns shown in Fig. 9 and those in Fig. 10 when appropriately oriented relative to one another. The resulting complications and ambiguities were taken into account in deducing the presence of the four preferred orientations indicated above. The occurrence of crystallites exhibiting preferred c-axis orientation parallel to $T$ indicates that the film was stretched to a greater extent parallel to $T$ than to $M$ in manufacture. The density of a sample of this tape was found to be $0.909{ }_{3}$ which corresponds to a weight fraction crystallinity of 0.69 .

\subsubsection{Nylon-11 (Nylon 11-A)}

Two different triclinic (see Glossary) unit cells have been proposed for Nylon-11 $(21,22)$. As background for our observations on the Nylon-11 tape it is of interest to note that according to slichter ${ }^{(22)}$ the side by side packing of the chain molecules and their arrangement in hydrogen bonded sheets in the Nylon-11 crystal lattice is similar to that found in the well established a-triclinic unit cells of Nylon 6.6 and Nylon $6.10^{(23)}$. The Nylon-11 unit cell parameters given by Slichter are $a=4.9 \AA, b=5.4 \AA, c=14.9 \AA, \alpha=49^{\circ}, \beta=77^{\circ}, \gamma=63^{\circ}$. One chain passes through this cell with its long axis parallel to the c-axis. These unit cell parameters are identical with those of Nylon 6.6 and Nylon 6.10 except that the c-axis is shorter in accordance with the difference in length between the w-amino undecanoic acid repeat unit in Nylon-11 and the diamine-dioic acid repeat units in 
Nylon 6.6 and Nylon 6.10. As is the case for the latter two polymers, one would expect WAXD patterns of unoriented crystalline Nylon-11 to exhibit in particular, two characteristic intense rings, one of which corresponds to diffraction from the (100) planes and the other, which is a composite ring, corresponds to diffraction from the (010) and (110) planes in the crystallites. The calculated interplanar spacings are $d_{100}=4.36 \AA, d_{110}=3.7 \AA$ and $d_{010}=3.67 \AA$. It is pertinent to note in anticipation of what follows that the (100), (010) and (110) spacings (which define the lateral packing of the chains) are known to vary in the case of Nylon 6.6 and Nylon 6.10, according to the degree of crystallinity of the polymers ${ }^{(24-27)}$. Thus, in rapidly quenched samples which have very low crystallinity, a single broad diffraction ring replaces the two above mentioned rings. Upon annealing such samples at elevated temperatures the broad ring resolves itself into two rings whose corresponding spacings approach the (100) and (010)(110) interplanar spacings in the $\alpha-$ triclinic cell as the annealing temperature and hence the crystallinity is increased.

The WAXD patterns obtained from the Nylon-11 tape showed no indication of crystalline orientation. In fact, as shown in Fig. 15a which was taken with the x-ray beam parallel to $\mathrm{N}$, they exhibited a broad diffraction ring corresponding to a spacing of 4.14A. Based on the previous experience with Nylon 6.6 and Nylon 6.10 summarized above, this feature was taken as evidence of the prevalence of poor crystalline order (crystallinity) in the tape. The conclusion was confirmed by the fact that a WAXD pattern obtained from the tape after it was annealed at $433 \mathrm{~K}$ for two hours, exhibited two distinct rings as may be seen in Fig. 15b. The spacings corresponding to these rings are $4.37 \AA$ and $3.86 \AA$. In view of the fact that Nylon-11 is currently not being considered as a prime candidate for use in the superconducing cable we have discontinued work on this tape and Nylon-11 generally for the present. The apparent existence of two crystal forms of Nylon-11 $(21,22)$ is an aspect which needs to be examined further in any future work on tapes of this polymer. 
M

a)

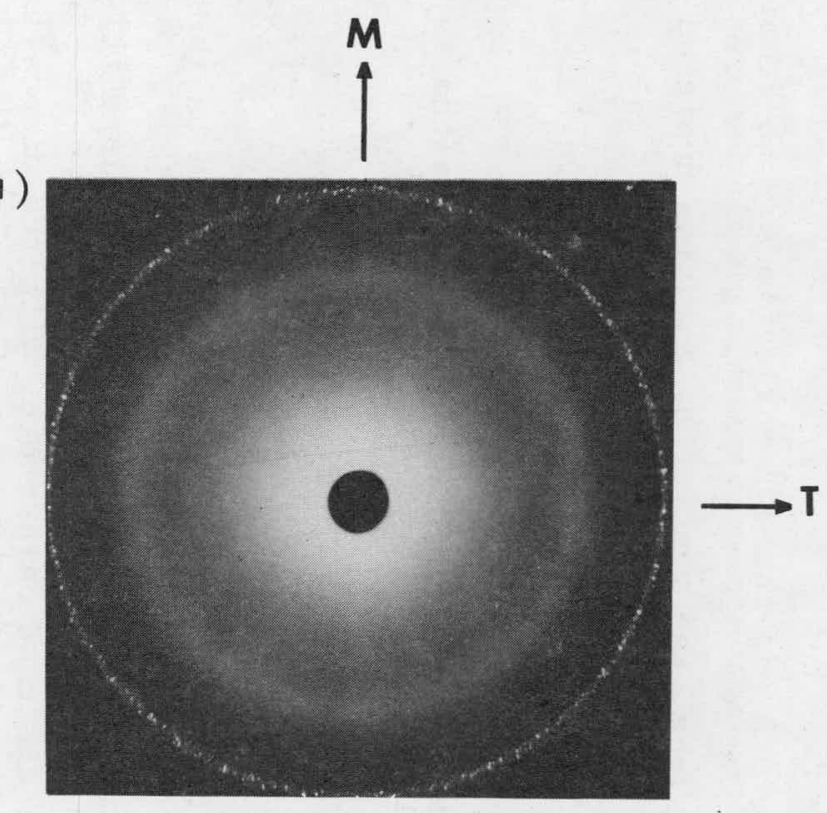

M

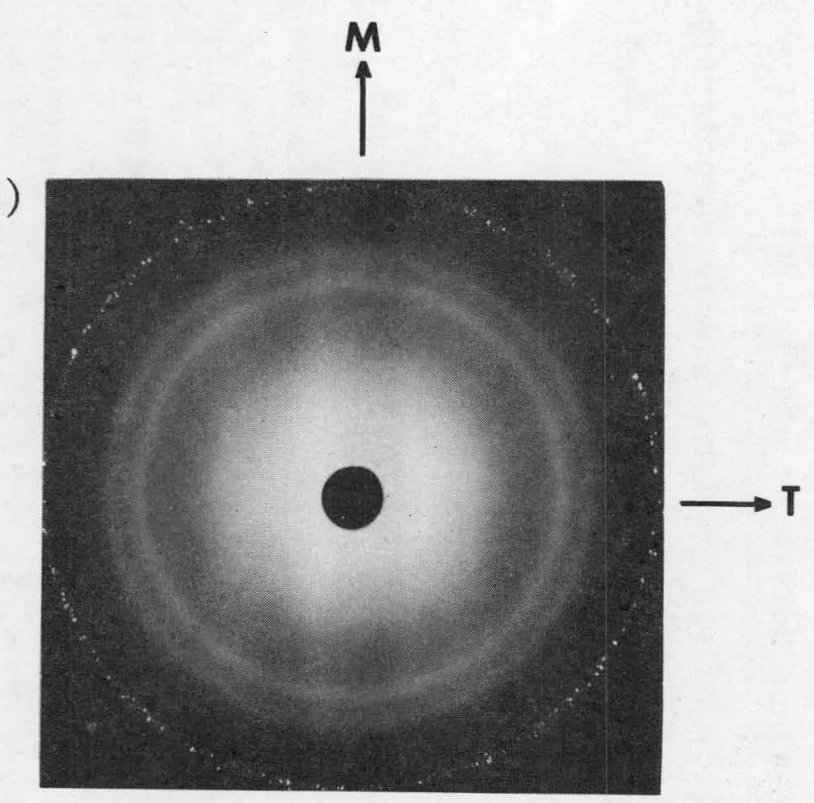

Figure 15: (a) N-axis WAXD pattern from the Nylon-11-A tape. (b) N-axis WAXD pattern from the same tape after annealing at $433 \mathrm{~K}$ for two hours. The spotty outer ring in each pattern is the 111 ring from polycrystalline $\mathrm{Si}$ used for calibrating the specimen to film distance. 


\subsubsection{Polycarbonate Tapes}

Two types of tapes made from poly (4, 4'-dioxydipheny $1-2,2^{\prime}$-propane carbonate) were examined, namely Polycarbonate-A and Polycarbonate-B. Both tapes were dyed in manufacture. The polymer will be referred to below simply as polycarbonate.
According to Bonart
this polycarbonate has a monoclinic unit cell. Its parameters are $a=12.3 \AA, b=10.1 \AA, c=20.8 \AA, \gamma=84^{\circ}$. Four chains pass through the cell with the chain axes parallel to the c-axis.

As an aid to the ensuing discussion of the WAXD patterns obtained from the tapes we show in Fig. 16 the calculated dispositions of some of the more intense reflections expected in a WAXD pattern from a polycarbonate film in which the crystallites are oriented with the c-axis parallel to the machine direction $\mathrm{M}$, their orientations being otherwise randomized in a cylindrically symmetrical manner about M. The reflections which are shown correspond to some of the most intense ones observed experimentally by Bonart in uniaxially stretched samples, in which case the most intense ones were the 020 and the 202 reflections. In keeping with the nomenclature used for polypropylene, the type of orientation depicted in Fig. 16 will be referred to as c-axis orientation paralle1 to M.

\subsection{4a Polycarbonate-A}

The M-axis, T-axis and N-axis WAXD patterns obtained from this tape are shown in Figs. 17a, b, and c respectively.

The WAXD pattern obtained with the $\mathrm{x}$-ray beam parallel to $\mathrm{N}$ (Fig. 17c) exhibited arcs which could be indexed 101, 103, 202, 203, 213, and 303 based on their corresponding interplanar spacings. Furthermore, a comparison of the dispositions of these arcs relative to the M-axis in the diffraction pattern, with the dispositions of the same reflections in the calculated pattern illustrated in Fig. 16 showed that they were indeed similar to one another. Particularly conspicuous features were the occurrence of the 101, 202, and 303 arcs along diameters oriented at very close 


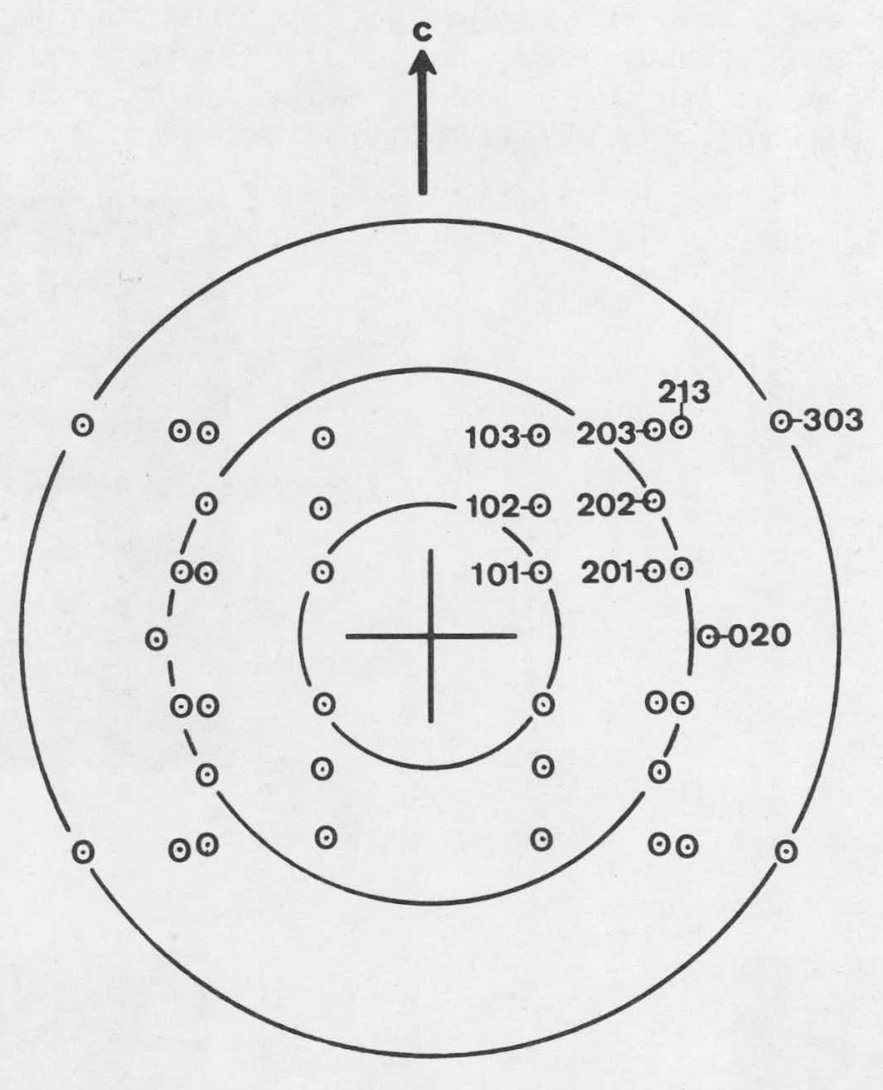

Figure 16: Calculated dispositions of the 101, 102, 103, 201, 202, 020, 203, 213, 303 ref lections in a WAXD pattern of a polycarbonate film having c-axis orientation. The crystallites are oriented with their c-axis parallel to the vertical direction in the diagram, their orientations are otherwise randomized in a cylindrically symmetrical fashion about that direction. X-ray beam is normal to
the plane of the figure. 
a)
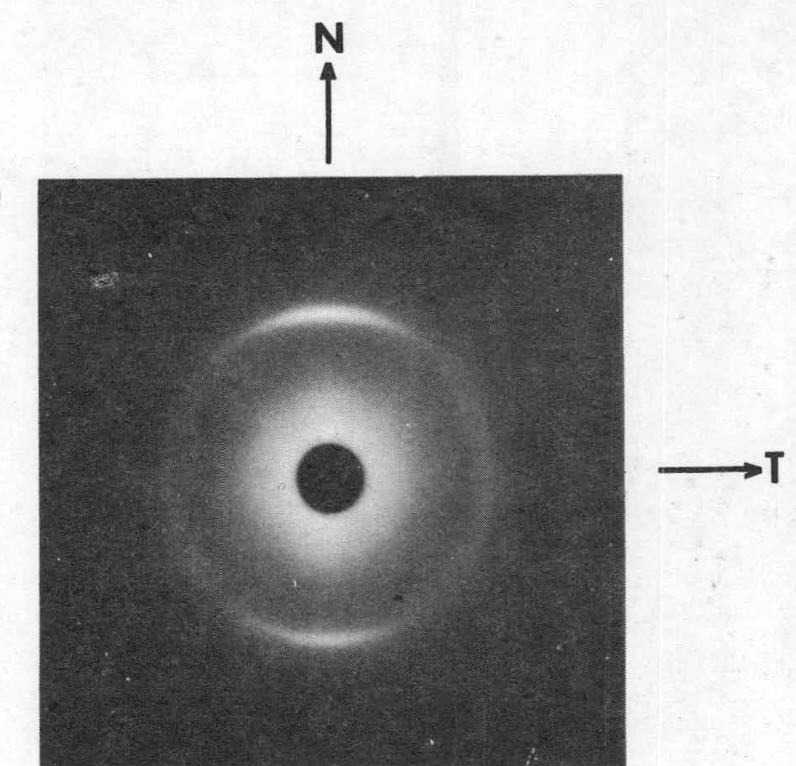

c)

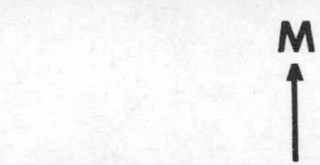

M

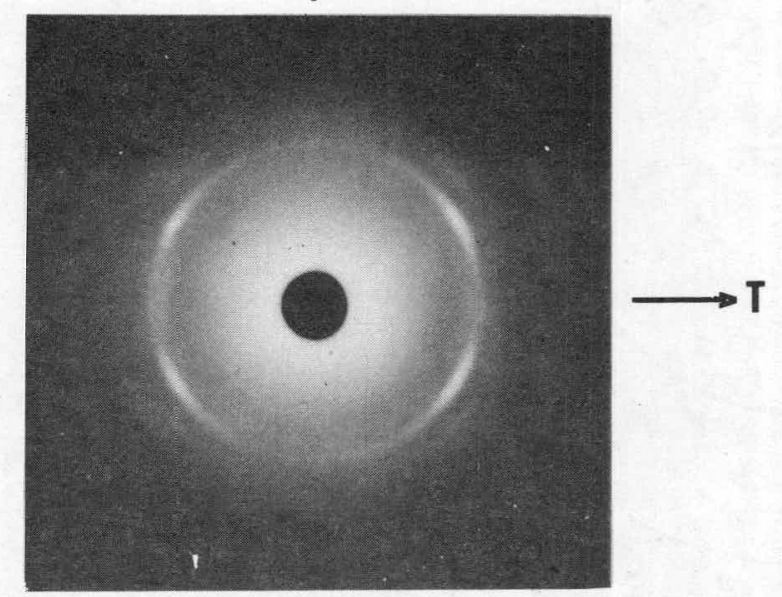

b)

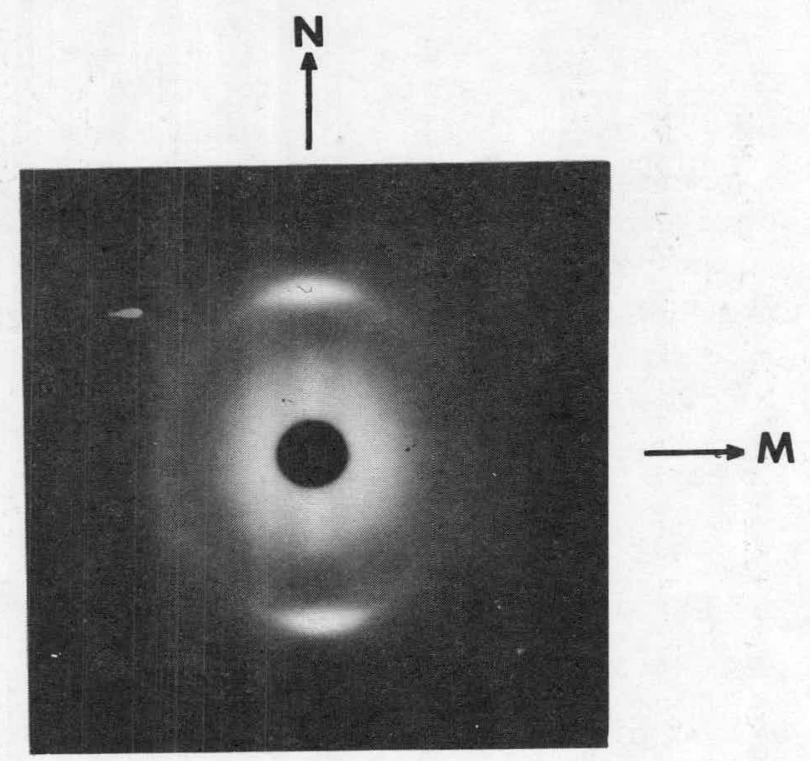

d)

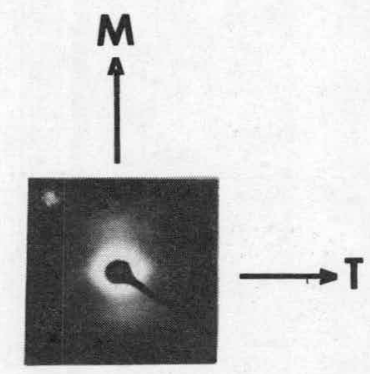

Figure 17: (a) M-axis, (b) T-axis, (c) N-axis, WAXD patterns from Polycarbonate-A tape. Contact prints of originals, specimen to film distances are not identical. The strong arcs situated along $\mathrm{N}$ in (a) and (b) are 020 reflections. In (c) the most intense arcs situated along diameters at about $+60^{\circ}$ to $M$ are 202 reflections. (d) N-axis SAXD pattern from the same tape. 
to $\pm 60^{\circ}$ relative to $\mathrm{M}$, and that the 202 arcs were the most intense - they are the most prominent ones in Fig. 17c. The other arcs were considerably fainter and are somewhat difficult to distinguish in Fig. 17c, in particular the 101 arcs which are overshadowed by the scattering around the central beam stop. All these weaker reflections were however discernable in the original photographic film.

The above mentioned coincidence between the calculated dispositions of the 101, 202, 303, 103, 203, and 213 reflections and the experimentally observed ones in the $\mathrm{N}$-axis diffraction pattern, leads to the conclusion that the crystallites in the tape are oriented with the c-axis preferentially parallel to M. There is, however, a feature which is conspicuous by its absence in the experimental N-axis WAXD pattern (Fig. 17c), namely the absence of 020 arcs. As may be seen from Fig. 16, 020 reflections would have been expected to occur along $\mathrm{T}$ in Fig. 17c, and would also have been expected to be as intense as the 202 reflections, if the preferred orientations of the crystallites in the tape corresponded to the cylindrically symmetrical c-axis orientation parallel to $\mathrm{M}$ represented in Fig. 16. In short, the absence of 020 reflections along $\mathrm{T}$ in the $\mathrm{N}$-axis WAXD pattern, taken together with the evidence obtained from the same pattern which indicates that the crystallites are oriented with their c-axis preferentially parallel to $M$, suggest the manifestation of some preferential orientation of the (020) planes relative to the surface (i.e., the MT plane) of the tape. This is substantiated by the WAXD pattern obtained with the x-ray beam parallel to M (Fig. 17a) in which the most prominent diagnostic feature is the occurrence of intense 020 arcs along $N$ indicating that the $(020)$ planes in the crystallies tend to be preferentially oriented parallel to the plane MT of the tape. It may also be noted that the T-axis WAXD pattern (Fig. 17b) exhibits 020 arcs along $\mathrm{N}$, and is also characterized by the absence of 101, 202, and 303 reflections along diameters at $\pm 60^{\circ}$ to $\mathrm{M}$. Both of these features are consistent with the deductions summarized above. 
A SAXD pattern taken with the $x$-ray beam paralle1 to $\mathrm{N}$ is shown in Fig. $17 \mathrm{~d}$. It exhibits distinguishable if diffuse diffraction spots situated along $M$ and corresponding to a periodic spacing of about $170 \AA$. These features point to the occurrence of chain folding in the crystallites, to the orientation of the fold surfaces at right angles to $M$, and to the orientation of the c-axis in the crysta1lites preferentially parallel to $M$. This latter aspect is in agreement with the WAXD results concerning the orientation of the c-axis of the crystallites.

In summary, the WAXD diffraction patterns obtained from this tape indicate that the crystallites are oriented with their c-axis (and hence their constituent chains) preferentially parallel to $\mathrm{M}$, and that the $(020)$ planes in the crystallites tend to be preferentially oriented parallel to the plane (MT) of the tape. The SAXD data which point to the manifestation of chain folding in the crystallites, substantiate the orientation of the c-axis in them preferentially parallel to $\mathrm{M}$.

\subsection{4b Polycarbonate-B}

The WAXD patterns obtained from this tape exhibited no distinguishable crystalline orientation. Indeed, as illustrated in Fig. 18a which is a pattern obtained with the $x$-ray beam parallel to $N$, the patterns were characterized by the occurrence of a diffuse halo indicative of poor crystalline order and hence very low crysta1linity. The spacing corresponding to this halo is about $5.3 \AA$. After annealing the tape at $473 \mathrm{~K}$ for twenty hours a sharper diffraction pattern indicating increased crystallinity was obtained. Such a pattern is illustrated in Fig. 18b in which the inner and most intense ring corresponds to a spacing of $5.08 \AA$, which agrees closely with the (020) interplanar spacing, of $5.02 \AA$, calculated on the basis of Bonart's unit cell parameters ${ }^{(28)}$. We have not investigated the effects of annealing this tape at higher temperatures on WAXD patterns in order to characterize the changes in the patterns as a function of crystallinity. It was considered sufficient, for the purpose of the present survey, and for qualitative comparison with the Polycarbonate-A tape, to have established the poor crystallinity and the lack of detectable crystalline orientation evidenced by the WAXD patterns of the Polycarbonate-B tape. 
a)
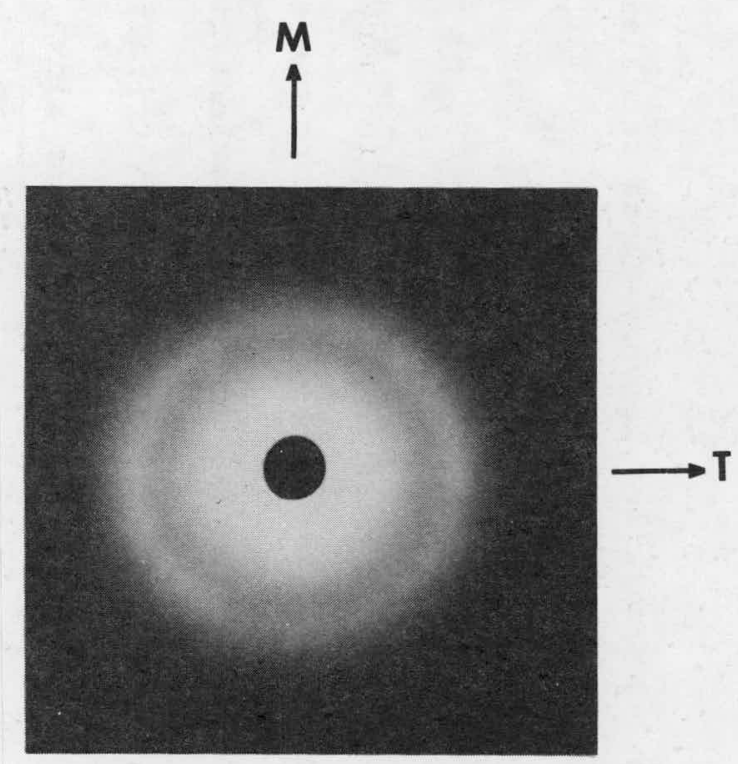

b )
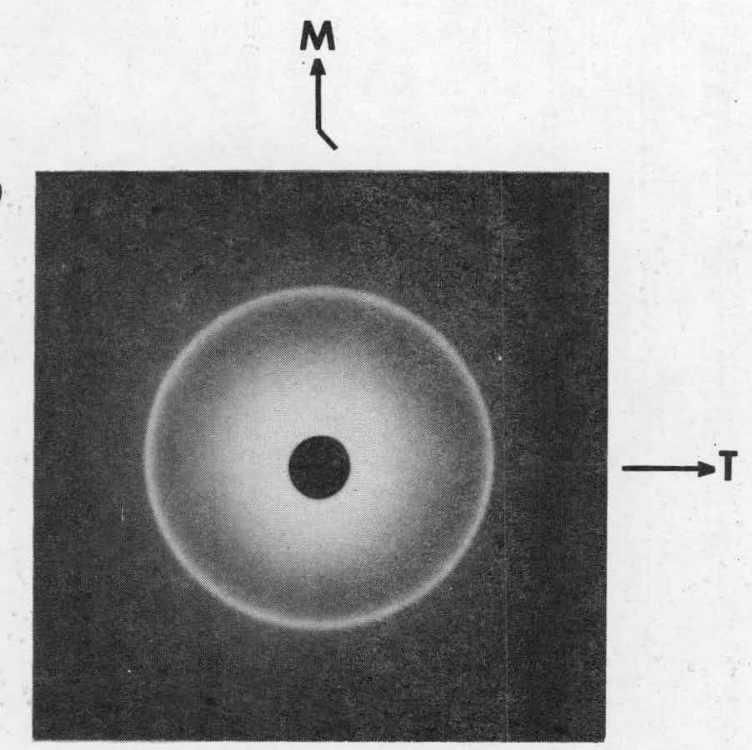

Figure 18: (a) N-axis WAXD pattern from the Polycarbonate-B tape. (b) N-axis WAXD pattern from the same tape after annealing at $473 \mathrm{~K}$ for twenty hours. Contact prints, specimen to film distances not the same. See text for comparison of patterns. 


\subsubsection{Polyethylene Tapes}

We report below some observations on the characteristics of crystalline orientation in the Polyethylene-A and the Polyethylene-D.

As background to the observations described below it may be first pointed out that the unit cell of polyethylene is orthorhombic (see Glossary). The unit cell parameters as determined by Bunn are $a=7.40 \AA, b=4.93 \AA, c=2.534 \AA^{(29)}$. Two chains pass through the unit cell and are parallel to the c-axis. Apart from the usual manifestation of preferential c-axis orientation (same nomenclature as used for polypropylene and polycarbonate) parallel to the stretching direction in the case of uniaxially stretched samples, a variety of preferred types of orientation have been achieved in polyethylene films or sheets by, for example, subjecting them to biaxial stretching ${ }^{(30)}$, rolling followed by heat relaxation ${ }^{(31)}$ or combinations of uniaxial stretching, rolling, and annealing (32).

\subsection{5a Polyethylene-A}

WAXD patterns taken with the $\mathrm{x}$-ray beam parallel or very close parallel to $\mathrm{M}, \mathrm{T}$ and $\mathrm{N}$ are shown in Figs. 19a, $\mathrm{b}$, and $\mathrm{c}$, respectively.

The fact that the N-axis diffraction pattern (Fig. 19c) was ringed in character, coupled with the fact that the positions of the diffraction arcs in the M-axis (Fig. 19a) and T-axis (Fig. 19b) patterns were essentially similar to one another, suggested at the outset the occurrence of a preferred crystalline orientation, or of preferred crystalline orientations, which are cylindrically symmetricaly about $N$. This indication, coupled with the occurrence of (200) and (110) arcs along $N$ in both the M-axis and the T-axis diffraction patterns suggested in turn the existence of two populations of crystallites in the tape, having preferential (200) planar orientation and $\{110\}$ planar orientations respectively (same nomenclature as for polypropylene Section 2.2.2). Such orientations have been previously reported by Adams $(30)$. 
a)

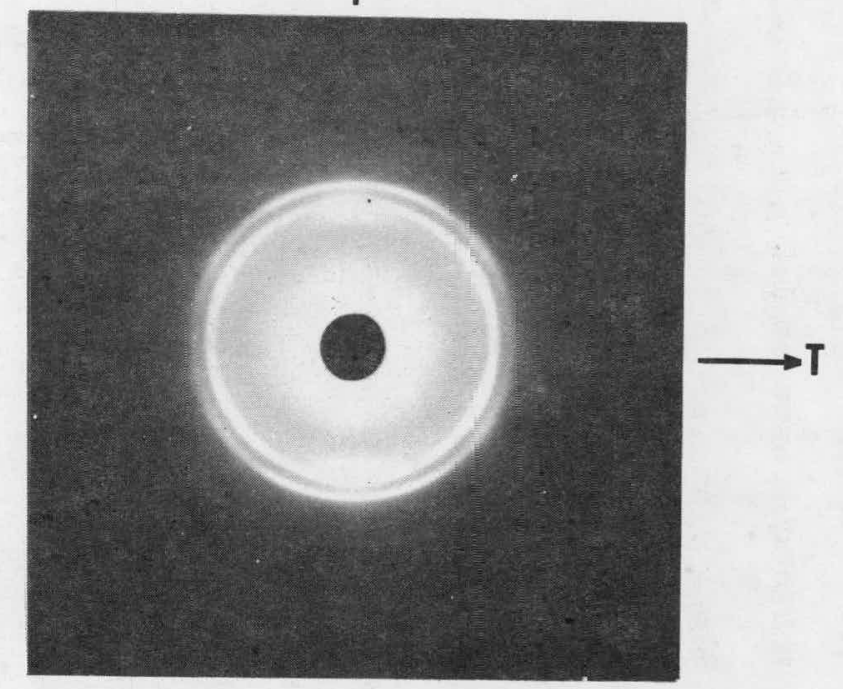

M

c)
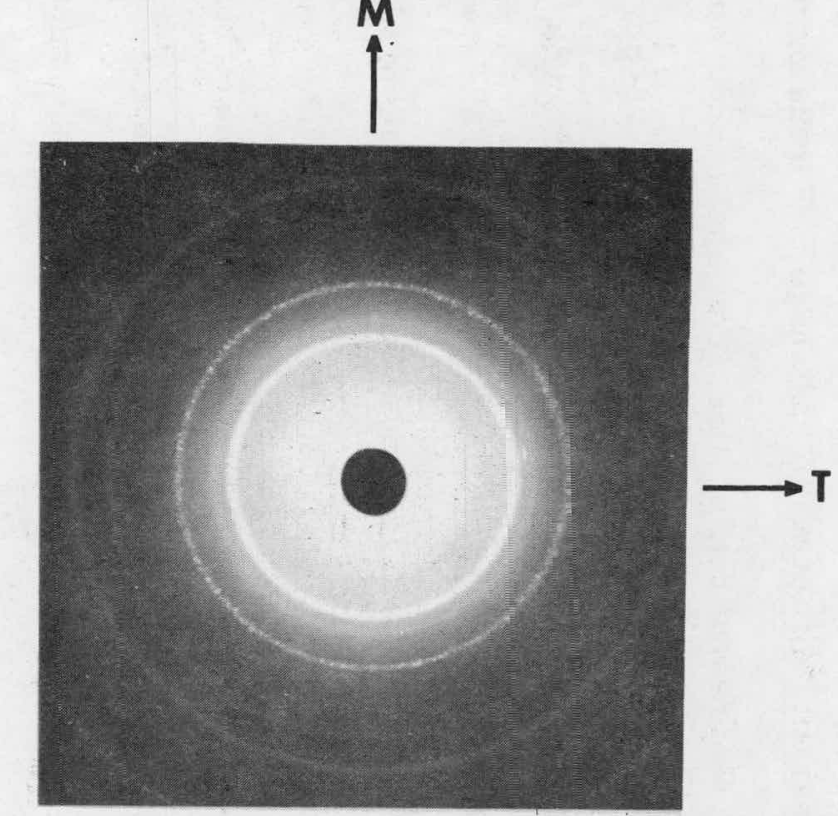

N

b)

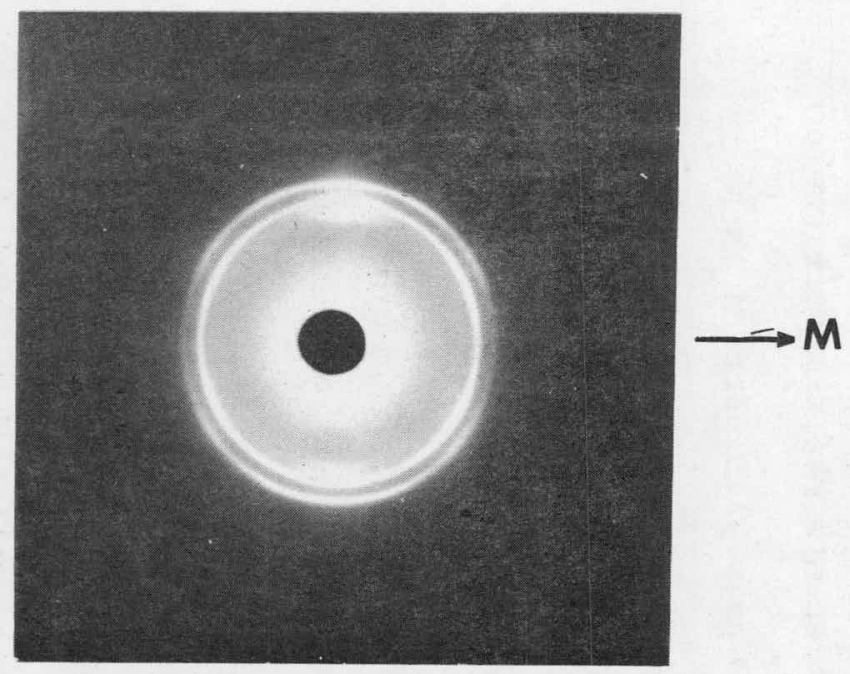

d)

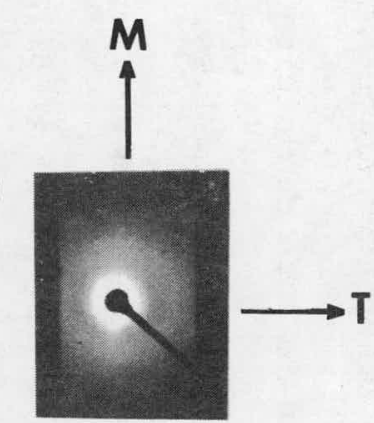

Figure 19: (a) M-axis, (b) T-axis, (c) N-exis, WAXD patterns from the Polyethylene-A tape. The spotty ring in (c) is the 111 ring from polycrystalline Si used for calibrating the specimen to film distance. The intense inner arc situated along $\mathrm{N}$ in (a) and (b) is a 110 reflection. The inner ring in (c) is a 110 ring. 
A comparison was made between the experimental M-axis and T-axis WAXD patterns and the pattern shown in Fig. 20 which represents the dispositions of the $110,200,210$, and 020 reflections in an $M$ or $T$ axis WAXD pattern of a hypothetical tape in which one population of crystallites exhibits (200) planar orientation and the other population exhibits $\{110\}$ planar orientation. It should be emphasized in this connection that the angular spreads of the arcs in the experimental WAXD patterns in Figs. 19a, and 19b, are large. This feature, coupled with uncertainties involved in estimating the positions of maximum intensity along the arcs, has made it difficult to analyze and compare these patterns rigorous ly with the calculated composite pattern in Fig. 20. We shall not enumerate or discuss these difficulties. We point out that the incidence in the experimental M-axis and T-axis diffraction patterns, of 200 arcs along $\mathrm{N}$, of 110 arcs along diameters at about $+56^{\circ}$ relative to $\mathrm{N}$, and of 020 arcs along $\mathrm{T}$ and $\mathrm{M}$ respectively, are consistent with the occurrence of a population of crystallites having preferential (200) planar orientation in the tape. Furthermore, the occurrence of 110 arcs along $\mathrm{N}$ and the manifestation of distinguishable 200 arcs along diameters at $\pm 50^{\circ}$ to $55^{\circ}$ relative to $\mathrm{N}$, and $(020)$ arcs along diameters at about $\pm 30^{\circ}$ to $\mathrm{N}$ are indicative of the occurrence of preferential $\{110\}$ planar orientation.

Additiona1 WAXD experiments involving the recording of diffraction patterns with the $x$-ray beam tilted systematically at different angles relative to the $M$, $\mathrm{N}$, and $\mathrm{T}$ axes should shed further light on the crystallographic orientations in this tape. The main features which we derive from the present WAXD experiments are that the c-axis and hence the chain molecules in the crystallites are oriented preferentially parallel to the plane (MT) of the tape and that the WAXD patterns do not reveal any preferential orientation of the c-axis about any direction in that 


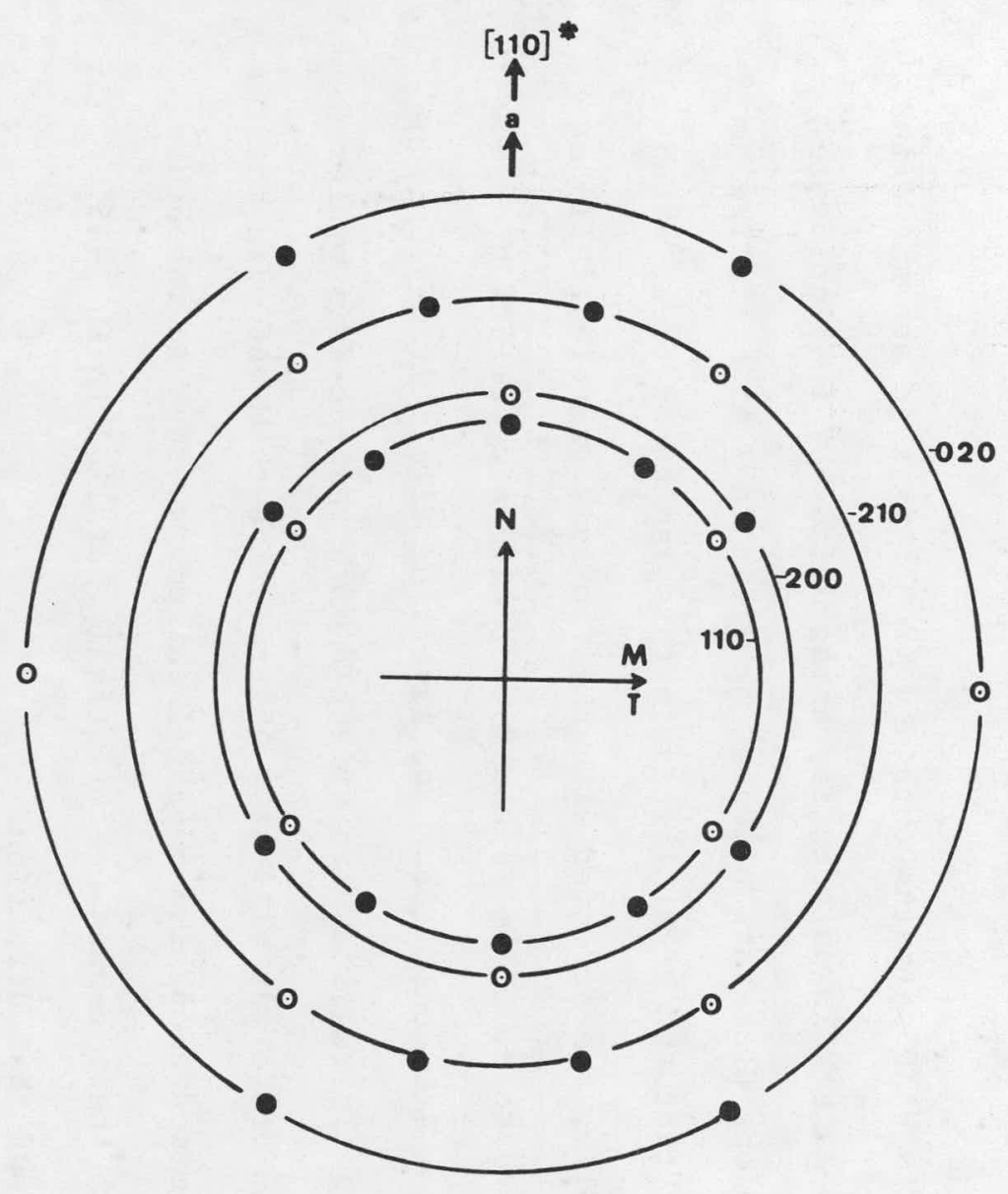

Figure 20: Calculated disposition of the $110,1 \overline{1} 0,200$, and 020 , reflections, in a wAXD pattern

film havin the $\mathrm{x}$-ray beam parallel to $\mathrm{M}$, $\mathrm{T}$, or any direction parallel to the MT plane in a polyethylene 200 lanar orient the planar orientation in which the crystallites have their (200) planes parallel to the MT plane of the film, the a-axis being at right angles to MT. The filled circles are the reflections corresponding to 110$\}$ planar orientation in which the crystallites have their $\{110\}$ planes paralle 1 to the MT plane and the [110]* or [110]* reciprocal lattice axes at right angles to MT. 
plane. A SAXD pattern obtained with the $\mathrm{x}$-ray beam parallel to $\mathrm{N}$ is shown in Fig. 19d. It exhibits a very diffuse diffraction ring which, in addition to indicating the presence of chain-folding in the constituent crystallites in the tape, also points to the absence of (or very poor) preferential orientation of the chain molecules in the crystallites relative to $\mathrm{M}$. The spacing corresponding to the diffraction ring in Fig. 19d is 200A. Finally, the density of a sample of this tape was 0.932 which corresponds to a weight fraction crystallinity of 0.57 .

\subsection{5b Polyethy lene-D}

M-axis, T-axis and $\mathrm{N}$-axis WAXD patterns obtained from this tape are shown in Figs. 2la, b, and c. The N-axis WAXD pattern is characterized by the incidence of 110,200 , and 020 arcs along diameters oriented at close to $\pm 45^{\circ}$ relative to M. These features indicate that the crystallites in this laminated tape, which consists of two layers of film, are oriented with their c-axis preferentially at either $+45^{\circ}$ or $-45^{\circ}$ relative to $\mathrm{M}$. This is consistent with a description of how this tape is constructed from its constituent two layers of film. It may be concluded that in one layer the crystallites are oriented with their c-axis preferentially in the (MT) plane and preferentially at $+45^{\circ}$ relative to $M$ but with their orientation otherwise randomized about the $+45^{\circ}$ direction, whereas in the other layer they are oriented with their c-axis preferentially parallel to the $-45^{\circ}$ directio and randomized about that direction.

A SAXD pattern obtained with the $\mathrm{x}$-ray beam parallel to $\mathrm{N}$ is shown in Fig. 21d. No discrete reflections were observed. The continuous scatter around the beam stop is almost square-like in appearance with the corners of the squares situated at $\pm 45^{\circ}$ to M. This feature can be interpreted as indicating the presence of elongated microvoids oriented with their long dimension at $\pm 45^{\circ}$ to $M$. The manifestation of fourfold symmetry in the scatter around the beam stop is consistent with the two preferred orientations indicated above. 
a)
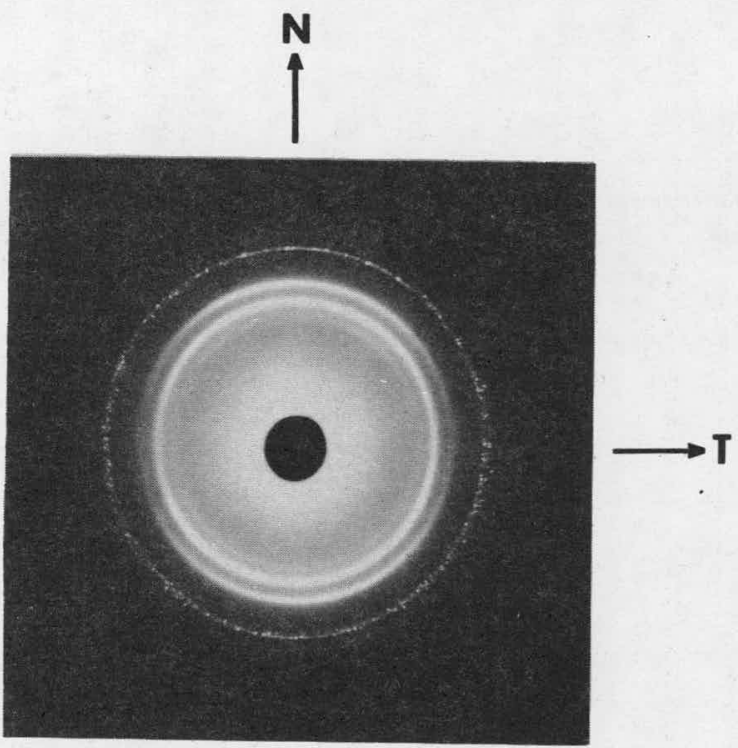

C )

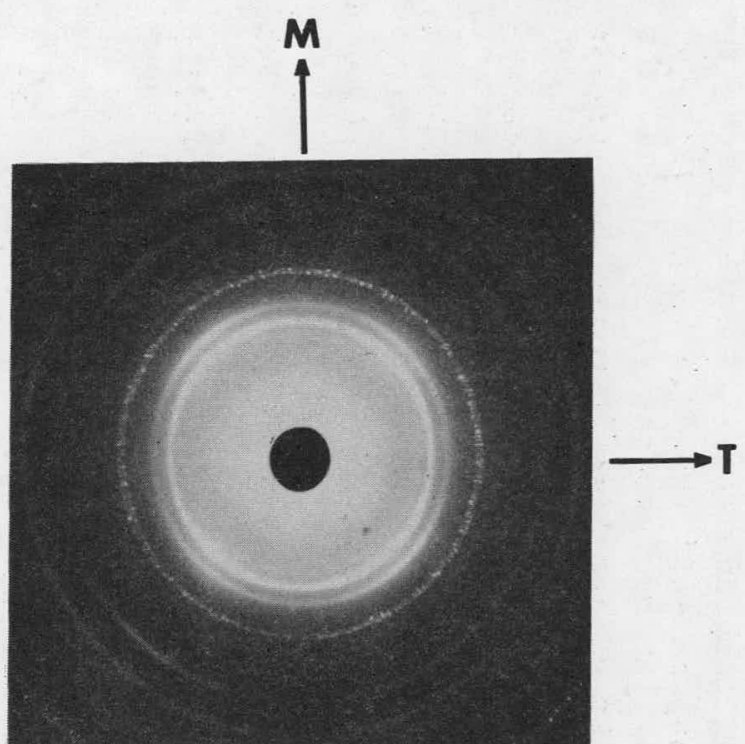

b)

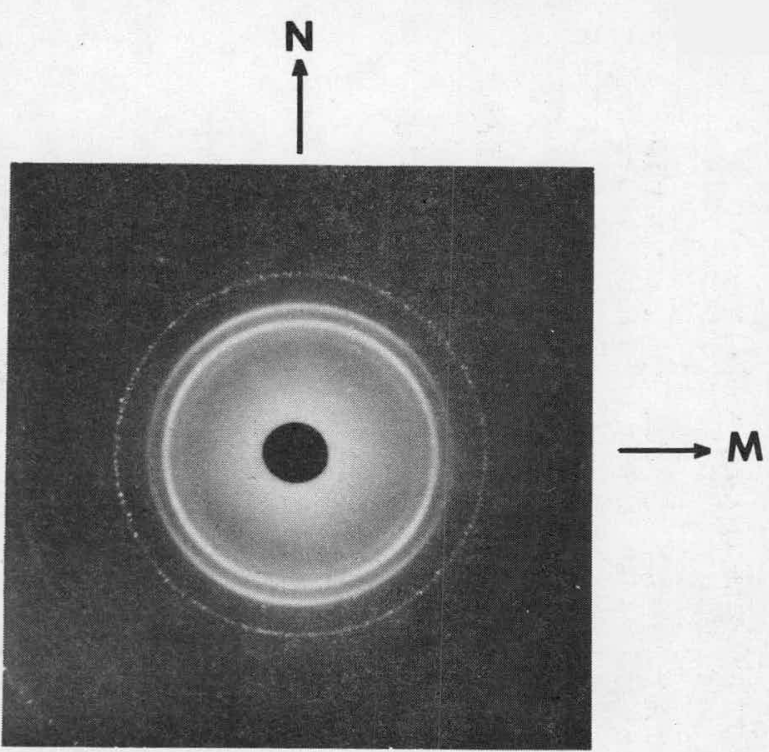

d)

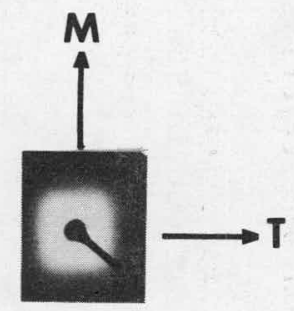

Figure 21: (a) M-axis, (b) T-axis, and (c) N-axis WAXD patterns from the Polyethylene-D tape. The spotty ring in each pattern is the 111 ring from polycrystalline $S i$ used for calibrating the specimen to film distance. (d) $\mathrm{N}$-axis SAXD pattern from the same tape. 


\subsubsection{Discussion and Summary}

Using primarily WAXD as a probe and SAXD as an ancillary tool, a qualitative survey has been carried out of the crystalline orientation in the following tapes: Polypropylene $(A, B, C, D, E)$, Nylon 11, (Nylon 11-A). Polycarbonate $(A, B)$, Polyethylene $(A, D)$.

It has been pointed out in Sections (1.3.2), (1.3.4) and (1.3.5) that the Nylon 11, the polyethylene, and the polypropylene tapes meet the established riteria in so far as their dielectric properties in the range 6-8 $\mathrm{K}$ are concerned. They are considered unsuitable however with regard to at least one aspect of their mechanical properties ${ }^{(33)}$. This aspect is considered below in conjunction with our observations on the structural characteristics of these tapes.

The matter of what are the desirable mechanical properties which polymer tapes must have is a complex problem which remains to be fully resolved in so far as establishing comprehensive criteria. Among the factors which are to be considered are the tensile modulus, the yield stress, the elongation to break, the breaking strength, creep and stress relaxation, and non-linear viscoelastic characteristics. These properties which are structure dependent, and which are interelated, are of interest not only at cryogenic temperatures, but also at room temperature. The interest in this latter case is in connection with the tape winding process used in the manufacture of the superconducting cables. The current view is that the tensile modulus $\mathrm{E}$ of the tapes at room temperature and in the machine direction $\mathrm{M}$ should be $5 \times 10^{5}-6 \times 10^{5}$ psi $(3.4-4.1 \mathrm{GPa})$ or greater ${ }^{(33)}$, as determined under testing conditions adopted at $\mathrm{BNL}^{(34)}$. In addition the compressibility of the tapes is a factor whose importance to the success of the tape winding operation is currently receiving much attention. 
Confining our remarks to the tensile modulus $\mathrm{E}$ (parallel to M-axis) of the tapes at room temperature, it is important to bear in mind at the outset that there are limitations to using the magnitude of $\mathrm{E}$ obtained under one set of testing conditions as a general performance criterion. Not withstanding these limitations which have been outlined in a recent communication ${ }^{(35)}$ and will not be repeated here, data on $\mathrm{E}$ derived from a single set of testing conditions (34) (specimens $12.7 \mathrm{~cm}$ long, $1.9 \mathrm{~cm}$ wide, cross-head speed $1.27 \mathrm{~cm} / \mathrm{min}$. temp. $293 \mathrm{~K}$ ) provide a basis for comparison relative to the tentative criterion of $\mathrm{E}=5 \times 10^{5}-$ $6 \times 10^{5}$ psi $(3.4-4.1 \mathrm{GPa})$.

The tensile properties of the tapes which have been measured at BNL are listed in Table VIIa, from which it can be seen that the tensile moduli of the polypropylene, the Nylon 11, and the polyethylene tapes fall short of the above mentioned criterion. The respective structural characteristics of these tapes are certainly not favorable in this connection. It is sufficient in the present context to point out that among the features necessary for achieving a high tensile modulus are a high crystallinity, and the orientation of the crystalline regions (crystallites) with their c-axis (i.e. the chain molecules) parallel to the straining direction which is $M$ in the case of the tapes. The better the alignment of the c-axis in the crystallites parallel to that direction, the higher the tensile modulus. The polypropylene, the Nylon-11 and the polyethylene tapes fall short with respect to either one and/or the other of these features as can be judged from the summary given in Table VIIb of the qualitative WAXD results described in Sections (2.2.2) to 2.2.5). Finding commercial films, or alternatively, producing special films or tapes which have been subjected to high draw ratios in the machine direction $M$ during manufacture is clearly necessary if the criterion for $\mathrm{E}$ is to be met. A factor to bear in mind in this connection, however, is the tendency for samples having ultra-high c-axis orientation to fibrillate. 
In the case of the polycarbonate tapes, it has been pointed out in Section (1.3.1) that at $30 \mathrm{~K}$ and below the dielectric loss behavior of the Polycarbonate-A and the Polycarbonate-B are identical to one another (see Fig. 3) and do not meet the required specifications at the superconducting cable operating temperatures, 6-8 $\mathrm{k}$. In view of the evident fine structural differences between these two tapes as revealed by their WAXD characteristics and as summarized in Table VIIb (see also Sections 2.2.4a and 2.2.4b), it appears that this polymer is intrinsically unsuitable in so far as its dielectric properties are concerned. It may be noted that the structual differences between the two tapes are reflected in the distinct difference in intensity between their respective loss peaks at circa $180 \mathrm{~K}$. Furthermore, as would be expected, the tensile modulus of the crystalline and oriented Polycarbonate-A is higher than that of the poorly crystalline Polycarbonate-B (see TAble VII). Indeed, of the various tapes listed in Table VII, the Polycarbonate-A satisfies most closely the criterion for $E$, its use in the cables is negated, however, due to its dielectric properties.

Finally, although, as we have pointed out above, the polypropylene and the polyethylene tapes meet the required dielectric performance criteria at the superconducting cable operating temperatures, 6-8 $\mathrm{K}$, differences between dielectric loss levels were observed among tapes of each of these polymers in the range 4-10 $\mathrm{K}$. The origins of these differences remain obscure. It is at present not clear whether these differences are caused wholly due to differences in additive content or whether structural features are a contributing factor.

\subsection{Some Observations on the Surface Textures of Polymer Tapes}

Following some background remarks, we illustrate below a few initial observations on the surface textures of some polymer tapes. These observations point to the fact that opposite surfaces of some tapes exhibit different textural features on a coarse scale. In addition some of the examples which will be shown serve to illustrate diverse surface textural features encountered in different tapes. 
The long term objective of the study of the surface textures of promising or trial polymer tapes is to characterize them at different levels of resolution. The techniques which will be used include the application of optical microscopy and electron microscopy to examine replicas of the tape surfaces, and the application of scanning electron microscopy to examine the surfaces directly. The reason for undertaking such studies stems from interest at BNL in the following tape properties which are being investigated both at BNL and elsewhere, namely: (a) The frictional properties of the tapes, which are of interest in connection with the desired flexibility which the superconducting cables must have so that they can be wound around 8-10 ft. diameter reels for transportation to installation sites. (b) The radial thermal conductivity at cryogenic temperatures of the ( 0.5-1") thick layer of polymer tape insulation in the cables. This is of interest in connection with the problem of the dissipation of heat generated in the insulation due to dielectric loss. Knowledge of the nature of the interfacial contact between successive tape layers, and hence knowledge of the surface textures of the tapes is clearly relevant to both (a) and (b).

Our current observations on the surfaces of the tapes are presented below in the form of short notes.

\subsubsection{A coarse textural difference between opposite surfaces of the}

\section{Polypropylene-A Tape}

Fig. 22a and Fig. 22b are phase contrast light optical micrographs of replicas (36) of opposite surfaces of this tape. The particular features we wish to point out are the somewhat irregular, but roughly circular or oblong, outlines in the central part of Fig. 22a (see arrows) where three such features may be seen. These features were observed throughout this particular surface of the tape. The diameters of the circumscribed areas varied, the largest being $120 \mu \mathrm{m}$. No similar features were observed in replicas of the opposite surface of the tape as may be judged from Fig. 22b. 
a)

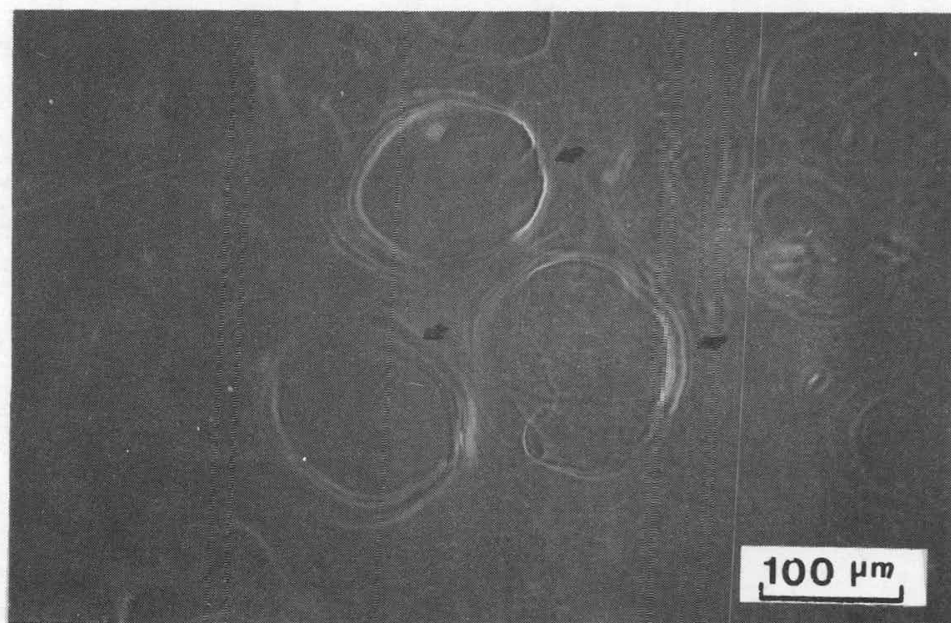

b)

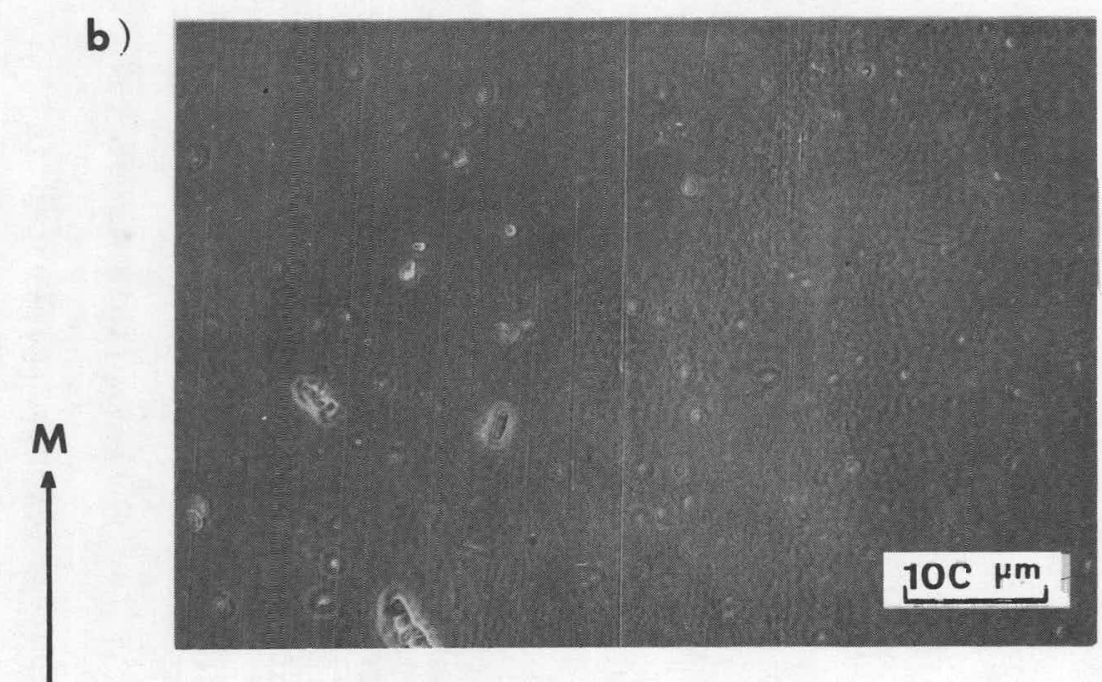

c)

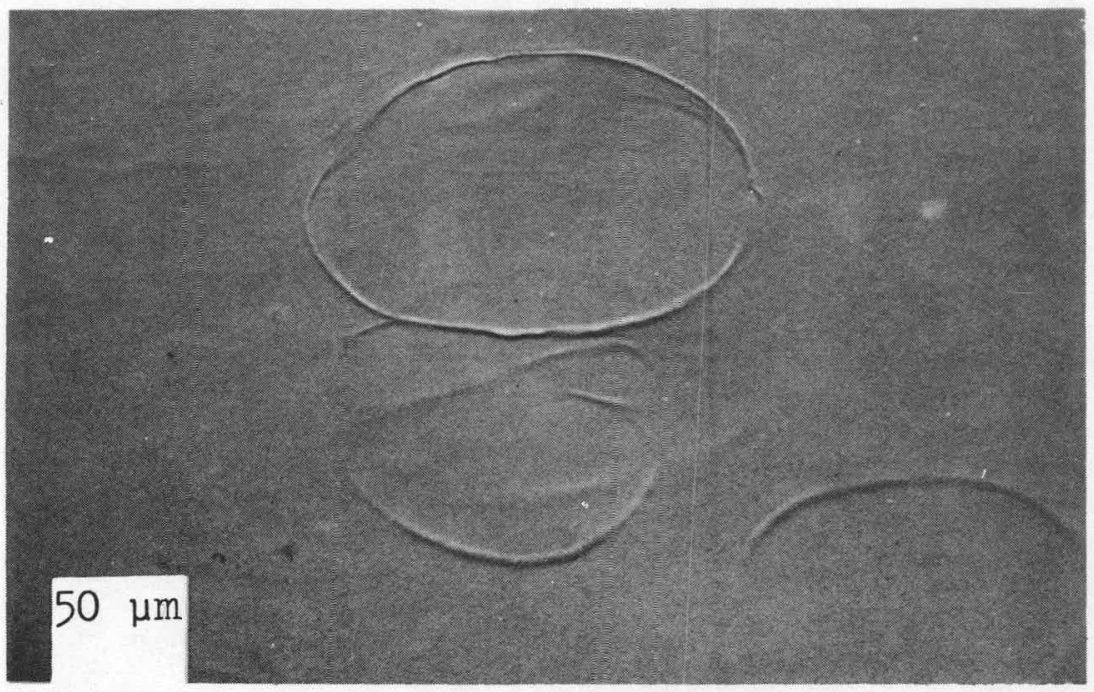

Figure 22:(a)Replica ${ }^{36}$ of one of the surfaces of the Polypropylene-A tape. (b) Replica of the opposite surface of the same tape. Phase contrast light optical micrcgraphs. (c) Scanning electron micrograph of the same surface of the tape as that which exhibits the features shown in (a). 
Fig. 22c is a scanning electron micrograph taken of a portion of the same tape surface ${ }^{(37)}$ as that whose replicas exhibit the type of features seen in Fig. 22a. It can be seen from Fig. 22c that the features observed in the replicas correspond to the occurrence of ridges which protrude from that tape surface. Detailed knowledge of the production procedures used in the manufacture of this $f i l m$ would shed some light on the origins of these ridges and their occurrence on only one side of the film. We point out parenthetically that examination of replicas of the Polypropylene-B tape under the optical microscope revealed the occurrence of similar ridges on only one side of that tape. No such ridges were observed in replicas of the Polypropylene-D or the Polypropylene-E tapes.

2.3.2 Texture differences between opposite surface of the Polycarbonate-A tape.

Fig. 23a and Fig. 23b are phase contrast light optical micrographs of replicas of opposite surfaces of this tape. The main feature we wish to point out in this case is the apparently cellular appearance of the area shown in Fig. 23a in which oblong outlines (see arrow) which are elongated in the direction parallel to $M$ can be seen. These bright outlines correspond to a difference in height at the surface of the tape relative to the surroundings. These features occur throughout this surface of the tape. They are absent, however, in replicas of the opposite surface as may be judged from Fig. 23b. Whether the outlines seen in Fig. 23a correspond to pronounced ridges at this surface of the tape remains to be determined.

It is also of interest to note the occurrence in Fig. 23b of lines which transverse the area shown in a variety of directions. Such lines which correspond to a difference in height relative to the surrounding area of the tape surface were seen in several replicas of this surface. No similar profusion of such lines was observed in replicas of the other surface as may be judged from Fig. 23a. 
a)

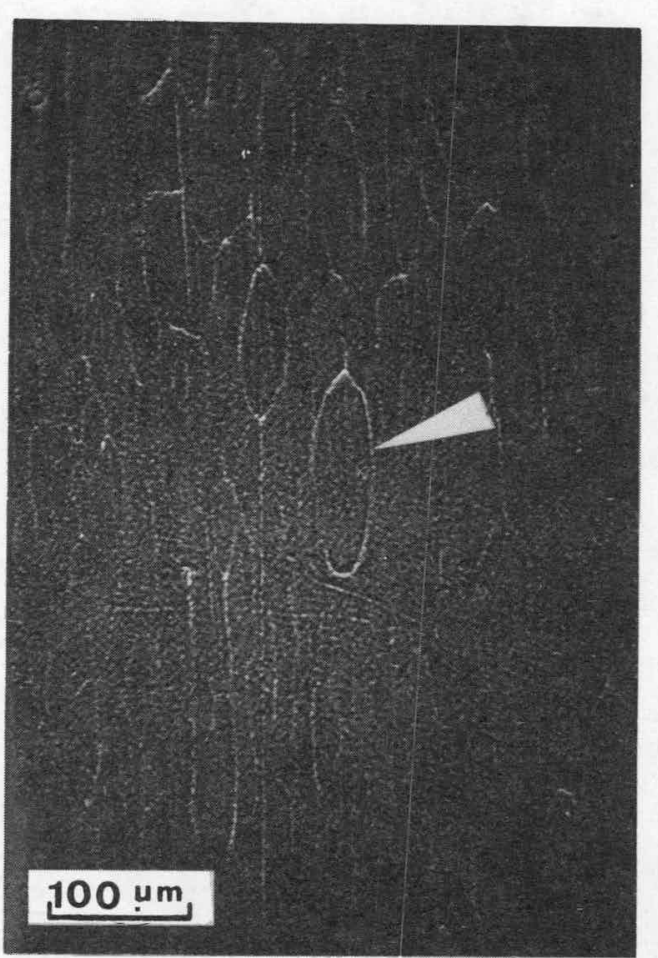

b)

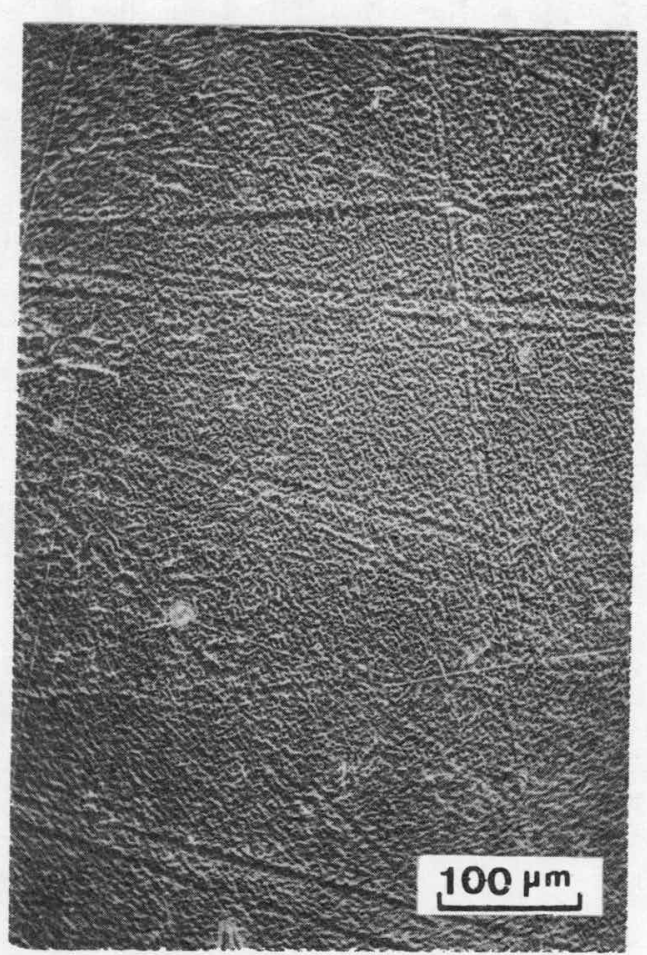

Figure 23: (a) Replica ${ }^{36}$ of one of the surfaces of the Polycarbonate-A tape. (b) Replica of the opposite surface of the same tape. Phase contrast light optical micrographs. 


\subsubsection{Texture differences between opposite surface of the Polyethylene-A tape.}

In this particular case differences between the opposite surfaces of the tape could be resolved under the phase contrast light optical microscope by focussing it on the opposite surfaces of the tape itself. Focussing on one particular surface always revealed a profusion of tree-branch like features exhibiting different configurations and extents of branching as may be seen in Fig. 24a (see arrows). Fig. 24b represents the same area of the tape as that shown in Fig. 24a, but with focussing on the opposite surface. The tree-branch like features seen in Fig. 24a are clearly out of focus in Fig. 24b in which a profusion of faint narrow lines running parallel to $M$ can be seen.

\subsubsection{Summary and Comment}

The main feature of the observations described in the previous Sections (2.3.1-2.3.3) on the surface textures of some of the polymer tapes is the manifestation of differences between opposite surfaces of those tapes. The differences which were pointed out may be described as being coarse in that they are readily detected at relatively low magnifications (e.g. x 160) in the light optical microscope using phase contrast optics. The term coarse, however, applies to the lateral extent of the features, their topographical characteristics remain to be examined in detail using scanning electron microscopy and the examination of surface replicas in the transmission electron microscope. This latter technique will also shed light on the finer aspects of the textures of the tape surfaces. 
a)

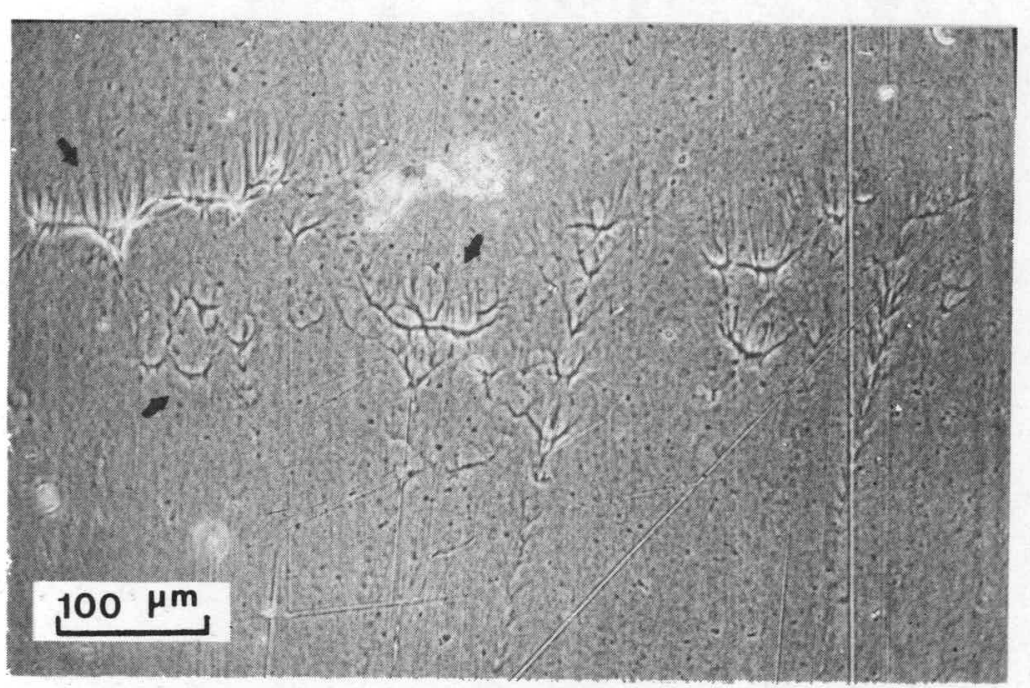

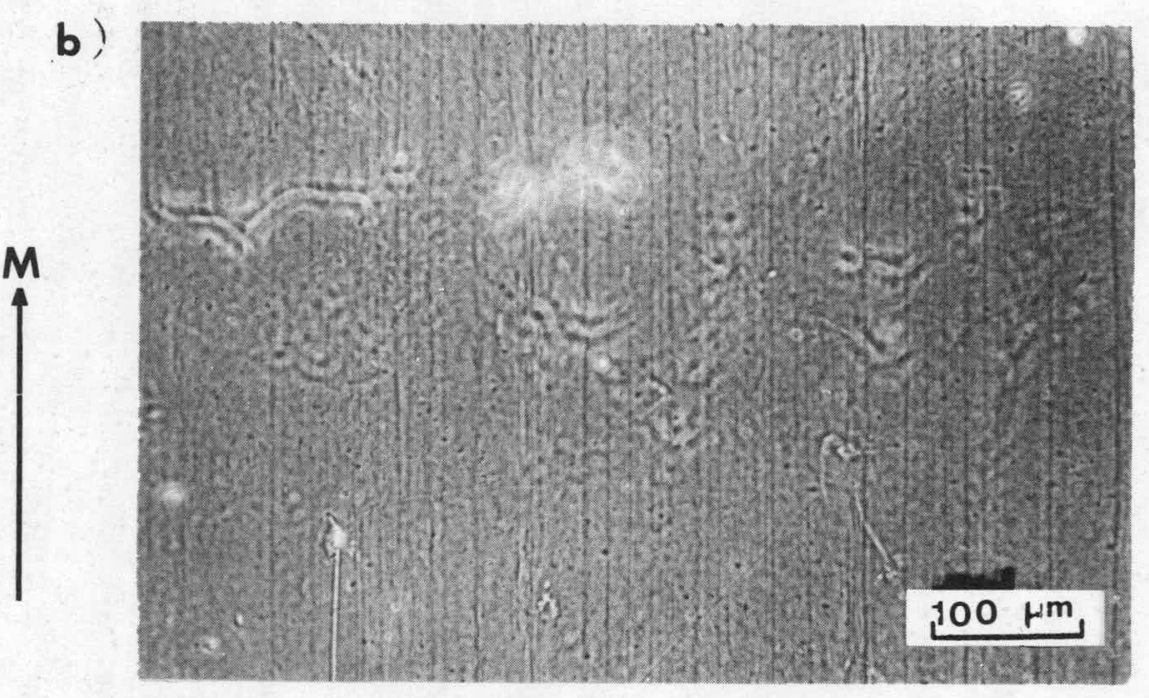

Figure 24: Phase contrast light optical micrographs of the same region in the Polyethylene-A tape. (a) Microscope focussed on upper surface of the tape. (b) Microscope focussed on lower surface of the tape. 


\section{References}

1. G. P. Nance and F. A. Sliemers, Summary Report to Brookhaven National Laboratory, Battelle Columbus Laboratories, Feb. 13, 1976.

2. A. Muller, Private Communication.

3. R. A. Thomas and C. N. King, App1. Phys. Lett. 26, 406 (1975).

W. A. Phillips, Proc. Roy. Soc. London, A $\underline{319}, \overline{565}$ (1970).

4. J. M. Crissman, J. A. Sauer, and A. E. Woodward, J: Polymer Sc1. A 3 , 5075 (1964).

5. The crystallization and fine structure (morphology) of crystalline polymers is discussed in the following two books: (a) P. H. Geil, "Polymer Single Crystals", Interscience Publishers, N. Y. (1963); (b) B. Wunderlich, "Macromolecular Physics", Vol. I (1973), Vol. II (1976), Academic Press, N. Y. For shorter reviews on the morphology of crystalline polymers see: (c) A. Keller, Reports on Progress in Physics 31, Part 2, 623 (1968). (d) $\bar{F}$. Khoury and E. Passaglia in, "Treatise on Solid State Chemistry", Vo1. 3, Chapt. 6, Plenum Press (1976). For models depicting crystalline orientation, chain folding, and tie molecules in uniaxially stretched polymers see: $(\underline{\epsilon}) \mathrm{R}$. Hoseman, Crit. Revs. Macromo1. Sci. 1 (No. 3), 351 (1972). (卢) A. Peterlin, J. Matls. Sci. $\underline{6}, 490$ (1971). (g) A: Peterlin, Text. Res. J., 42, 20 (1972).

6. Relationships between the fine structure and the physical properties of polymers are discussed in the following books:- (a) N. G. McCrum, B... E. Read, G. Williams, "Anelastic and Dielectric Effects in Polymeric Solids", Wiley. (1967); (b) I. M. Ward, "Mechanical Properties of Solid Polymers", Wiley 1971. Relationships between the fine structure of stretched samples, their mechanical properties, and the extent of stretching (draw ratio) have been reviewed by (c) D. M. Biggs, Battelle Columbus Laboratories Summary Report to BNL, Dec. 2nd (1975). Basic discussions on the elastic moduli of polymers may be found in the following papers: (d) I. Sakurada and K. Kaji, J. Polymer Sci. Part. C 31, 57 (1970); (e) L. Holliday and J. W. White, Pure and Appl. Chem. 26
599 (1975).

7. G. Natta and P. Corradini, Nuovo Cimento 15, (Supp1. No. 1), 40 (1960).

8. See for example International Tables for X-ray Crystallography. Volume II, p. 177 (Kynoch Press, 1959).

9. P. G. Andersen and S. M. Carr. J. Mat1s. Sci. 10, 870 (1975).

10. E. S. Clark and J. E. Spruiell, Polymer Preprints 16, (No. 2), 343 (1975).

11. F. Khoury, J. Res. Nat1. Bur. Stand. 70A, 29 (1966).

12. F. J. Padden and H. D. Kelth, J. Appl. Phys. 37, 4013 (1966).

13. F. J. Padden and H. D. Keith, J. Appl. Phys. 44, 1217 (1973).

14. S. Okajima, K. Homma, J. Appl. Polymer Sci. 12, 411 (1968). 
15. M. Kawai, Proceedings of the Fifth International Congress on Rheology Vol. 1 , p. 97 (S. Onogi Edit., University Park Press, Baltimore, 1969).

16. M. Wejo and S. Hoshino, J. Appl. Polymer Sc1. 14, 317 (1970).

17. G. C. Adams, Structure and Properties of Polymer Films, Polymer Science and Technology, Vol. 1, p. 169 (R. W. Lenz and R. S. Stein Edits., Plenum Press, N. Y. 1973).

18. Z. W. Wilshinsky, J. App1. Polymer Sci. Z, 923 (1963).

19. R. Kitamaru and S. H. Hyon, J. Polym. Sc1.: Polymer Phys. Ed. 13, 1085 (1975):

20. G. Natta, M. Peraldo and P. Corradin1, Stereoregular Polymers and Stereospecific Polymerization, Vol. 2. (F. Natla and F. Danusso Eds.) p. 600, Perganon. Press, 1967.

21. K. Little, Brit. J. App1. Phys. 10, 225 (1959).

22. W. P. Slichter, J. Polymer Sci. 36, 259 (1959).

23. C. W. Bunn and E. V. Garner, Proc. Royal Soc. (London) A189, 39 (1947).

24. P. F. Dismore and W. 0. Stattion, J. Polymer Sci. Part C 13, 133 (1966).

25. P. G. Stern, Kolloid Z. Z. Polymer, 215, 140 (1967).

26. M. Starkweather, Jr., J. F. Whitney and D. R. Johnson, J. Polymer Sci. A, 1 , 715 (1963).

27. I. Sandeman and A. Keller, J. Polymer Sc1., 19, 401 (1956).

28. R. Bonart, Makromol. Chem. 92, 149 (1966).

29. C. W. Bunn, Trans. Faraday Soc. $\underline{35}, 482$ (1939).

30. G. C. Adams, J. Polymer Sci. Part 2, 9, 1235 (1971).

31. I. L. May and A. Keller, J. Matls. Sc1. 1, 41 (1966).

32. J. J. Point, G. A. Homes, D. Gezovich, and A. Keller, J. Matls. Sc1. 4, 908 (1969).

33. A. Muller, private comrunication.

34. A. Muller, RGE, 84, 568 (1975), paper presented before the 12th Section of the Societe des Electriclens, des Electroniciens, et des Radio-electriciens.

35. Letter from F. Khoury, to A. Muller (BNL), May 21st (1976). 
36. The replicas are prepared as follows: A drop of a $10 \%$ solution of cellulose nitrate is deposited on the surface which is to be replicated. The solvent is allowed to evaporate at room temperature leaving behind a dry film of nitrocellulose whose side in contact with the tape is imprinted with the details of the topography of the tape surface in reverse. The nitrocelluliose replica is then gently stripped from the tape surface with tweezers and examined under the phase contrast light optical microscope.

37. As is common practice, the tape surface is coated with a thin layer $(-200 \AA)$ of evaporated gold prior to examination in the scanning electron microscope in order to prevent charging. 
Table I

Dielectric Constant and Loss at $100 \mathrm{~Hz}$ and $1 \mathrm{kHz}$

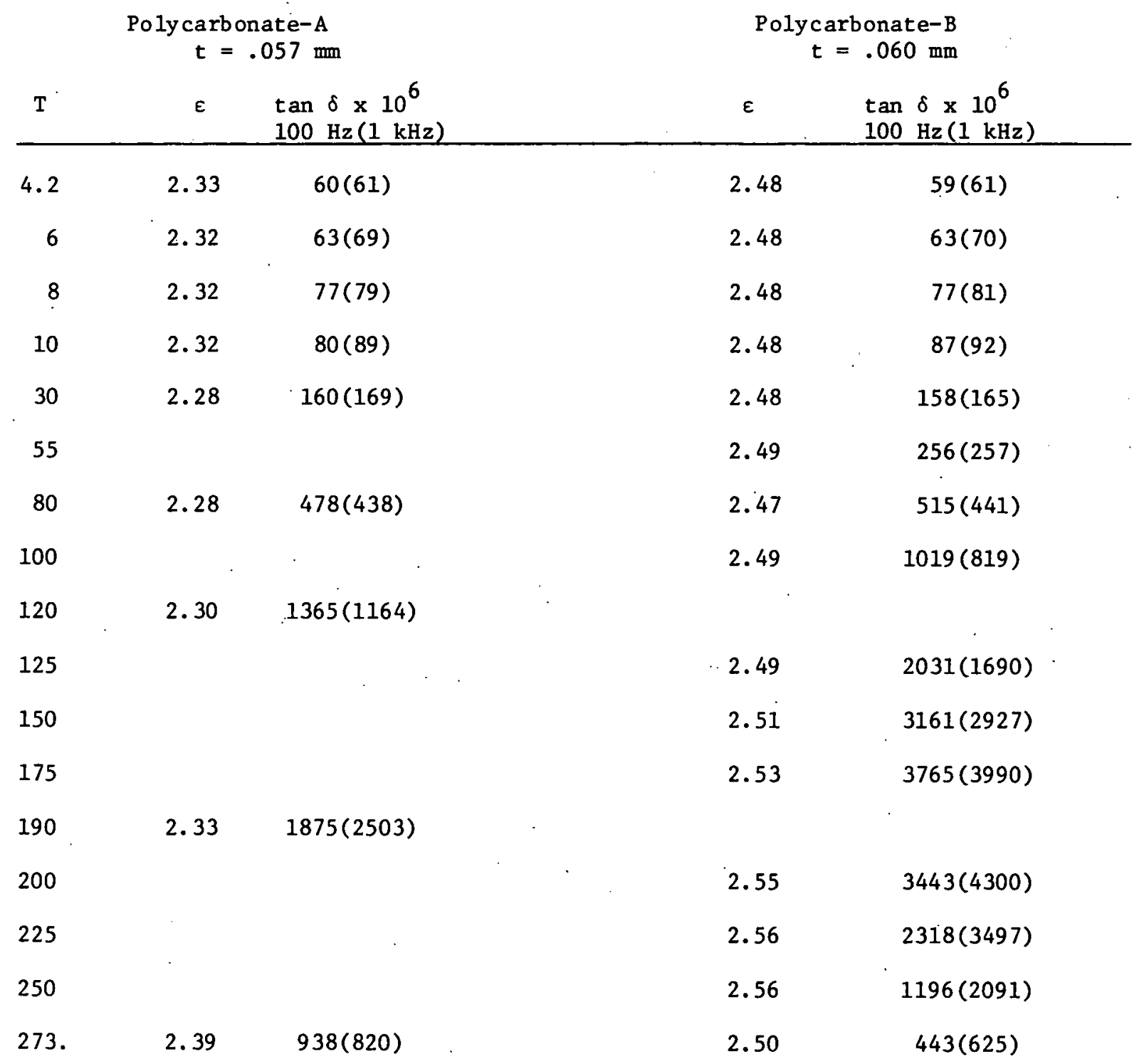




\section{Table II}

Dielectric Constant and Loss at $100 \mathrm{~Hz}$ and $1 \mathrm{kHz}$

\begin{tabular}{|c|c|c|c|c|}
\hline$T$ & $\begin{array}{l}\text { Nylon } 1 \\
t=.040\end{array}$ & $\begin{array}{r}\tan \delta \times 10^{6} \\
100 \mathrm{~Hz}(1 \mathrm{kHz}) \\
\end{array}$ & $\varepsilon$ & $\begin{array}{l}\text { fone-A } \\
53 \mathrm{~mm} \\
\tan \delta \times 10^{6} \\
100 \mathrm{~Hz}(1 \mathrm{kHz})\end{array}$ \\
\hline 4.2 & 2.52 & $20(23)$ & .2 .52 & $68(71)$ \\
\hline 6 & 2.52 & $16(21)$ & 2.51 & $80(85)$ \\
\hline 8 & 2.52 & $20(21)$ & 2.50 & $95(100)$ \\
\hline 10 & 2.52 & $18(20)$ & 2.51 & $113(110)$ \\
\hline 30 & 2.52 & $30(34)$ & 2.49 & $207(209)$ \\
\hline 80 & 2.52 & $296(247)$ & 2.49 & $528(524)$ \\
\hline 120 & 2.53 & $1729(1333)$ & 2.47 & $712(737)$ \\
\hline 190 & 2.67 & $10590(11750)$ & 2.42 & $815(822)$ \\
\hline 273 & 2.99 & $20330(19310)$ & 2.41 & $755(758)$ \\
\hline
\end{tabular}


Tab le III

Polyethylene-BNL Samples and Treatment History

BNL

No.

1

2

3

4

5

6

7

8

9

10

11

12

13

14
Antioxidant

As received

As received

$\mathrm{XX}$

$0.0492 \%$

$\mathrm{XX}$

$0.1024 \%$

$\mathrm{XX}$

$0.1024 \%$

$\mathrm{XX}$

$0.1024 \%$

$\mathrm{XX}$

$$
0.258 \%
$$

$\mathrm{XX}$

$0.5004 \%$

YY

$0.0488 \%$

YY

$0.1086 \%$

YY

$0.1086 \%$

$\mathrm{ZZ}$

$0.0492 \%$

ZZ

$0.1028 \%$

$\mathrm{ZZ}$

$0.1028 \%$
Extraction

Solvent

none

methano1 :

none

none

methanol

cyclohexane

none

none

none

none

-

methano 1

none

methanol

none 
Table IV

Dielectric Constant and Loss at $100 \mathrm{~Hz}$ and $1 \mathrm{kHz}$. Polyethylene

\begin{tabular}{|c|c|c|c|c|c|c|c|c|}
\hline \multicolumn{3}{|c|}{$\begin{array}{l}\text { Polye thy lene-A } \\
t=.026 \mathrm{~mm}\end{array}$} & \multicolumn{2}{|c|}{$\begin{array}{c}\text { Polyethylene-c } \\
t=.104 \mathrm{~mm}\end{array}$} & \multicolumn{2}{|c|}{$\begin{array}{l}\text { Polyethy lene-D } \\
t=.102 \mathrm{~mm}\end{array}$} & \multicolumn{2}{|c|}{$\begin{array}{l}\text { Polyethy lene-BNL-2 } \\
t=.170 \mathrm{~mm}\end{array}$} \\
\hline $\mathrm{T}$ & $\varepsilon$ & $\begin{array}{l}\tan \delta \times 10^{6} \\
100 \mathrm{~Hz}(1 \mathrm{kHz})\end{array}$ & $\varepsilon$ & $\begin{array}{l}\tan \delta \times 10^{6} \\
100 \mathrm{~Hz}(1 \mathrm{kHz})\end{array}$ & $\varepsilon$ & $\begin{array}{l}\tan \delta \times 10^{6} \\
100 \mathrm{~Hz}(1 \mathrm{kHz})\end{array}$ & $\varepsilon$ & $\begin{array}{l}\tan \delta \times 10^{6} \\
100 \mathrm{~Hz}(1 \mathrm{kHz})\end{array}$ \\
\hline 4.2 & 2.13 & $5(13)$ & 2.14 & $12(33)$ & 2.09 & $6(16)$ & 2.41 & $6(29)$ \\
\hline 6 & 2.12 & $3(12)$ & 2.14 & $9(26)$ & 2.09 & $4(12)$ & 2.41 & $3(13)$ \\
\hline 8 & 2.12 & $6(9)$ & 2.14 & $8(21)$ & 2.09 & $4(10)$ & 2.41 & $7(4)$ \\
\hline 10 & 2.12 & $5(8)$ & 2.14 & $5(18)$ & 2.09 & $6(9)$ & 2.41 & $5(6)$ \\
\hline 30 & 2.12 & $6(9)$ & 2.14 & $6(13)$ & 2.09 & $15(7)$ & 2.41 & $5(8)$. \\
\hline 55 & $2.11^{\circ}$ & $6(9)$ & $2: 13$ & $5(10)$ & 2.08 & $5(8)$ & 2.41 & $3(5)$ \\
\hline 80 & 2.10 & $13(15)$ & 2.13 & $12(12)$ & 2.08 & $10(12)$ & 2.40 & $4(7)$ \\
\hline 100 & 2.09 & $26(27)$ & 2.12 & $25(23)$ & 2.07 & $20(18)$ & 2.40 & $10(8)$ \\
\hline 125 & 2.07 & $44(41)$ & 2.11 & $42(46)$ & 2.07 & $42(37)$ & 2.38 & $17(16)$ \\
\hline 150 & 2.05 & $71(69)$ & 2.11 & $90(97)$ & 2.06 & $80(80)$ & 2.37 & $27(30)$ \\
\hline 175 & 2.03 & $61(82)$ & 2.10 & $102(145)$ & 2.05 & $98(118)$ & 2.36 & $22(33)$ \\
\hline 200 & 2.01 & $34(44)$ & 2.08 & $141(140)$ & 2.03 & $138(121)$ & 2.34 & $12(10)$ \\
\hline 225 & 1.98 & $32(36)$ & 2.07 & $187(213)$ & 2.02 & $202(203)$ & 2.33 & $6(6)$ \\
\hline 250 & 1.96 & $36(38)$ & 2.06 & $375(372)$ & 2.01 & $296(307)$ & 2.31 & $9(4)$ \\
\hline 273 & 1.93 & $51(52)$ & 2.04 & $365(459)$ & 1.99 & $268(331)$ & 2.30 & $6(7)$ \\
\hline
\end{tabular}


Table V

Loss Data Polyethylene-BNL Samples Tan $\delta \times 10^{6}$ at $100 \mathrm{~Hz}(1 \mathrm{kHz})$

\begin{tabular}{|c|c|c|c|c|c|c|}
\hline BNL & Tempera & & & & & \\
\hline No. & $4.2 \mathrm{~K}$ & $5.1 \mathrm{~K}$ & $6 \mathrm{~K}$ & $8 \mathrm{k}$ & $10 \mathrm{~K}$ & $30 \mathrm{~K}$ \\
\hline 1 & & 7 & & $4(21)$ & $(5(17)$ & $7(8)$ \\
\hline 2 & $6(29)$ & & $3(13)$ & $7(4)$ & $5(6)$ & $5(8)$ \\
\hline 3 & & 26 & & $11(48)$ & $9(36)$ & $0(16)$ \\
\hline 4 & & 16 & & $11(48)$ & $7(35)$ & $4(15)$ \\
\hline 5 & & 9 & & $28(18)$ & $7(12)$ & $0(7)$ \\
\hline 6 & & 2 & & $0(9)$ & $0(4)$ & $0(4)$ \\
\hline 7 & & 88 & & $4(214)$ & $21(165)$ & $0(62)$ \\
\hline 8 & $232(869)$ & & $126(636)$ & $73(448)$ & $50(332)$ & $20(129)$ \\
\hline 9 & $25(106)$ & & $17(70)$ & $15(47)$ & $9(34)$ & $5(15)$ \\
\hline 10 & $14(79)$ & & $5(47)$ & $19(34)$ & $2(23)$ & $2(14)$ \\
\hline 11. & $8(36)$ & & $6(13)$ & $2(3)$ & $2(2)$ & $5(1)$ \\
\hline 12 & $14(58)$ & & $10(32)$ & $0(19)$ & $0(14)$ & $6(8)$ \\
\hline 13 & $0(46)$ & & $0(21)$ & $O(13)$ & $1(6)$ & $3(5)$ \\
\hline 14 & $9(61)$ & & $9(32)$ & $5(21)$ & $9(11)$ & $8(5)$ \\
\hline
\end{tabular}


Tabıe VI

Dielectric Constant and Loss at $100 \mathrm{~Hz}$ and $1 \mathrm{kHz}$ Polypropylene

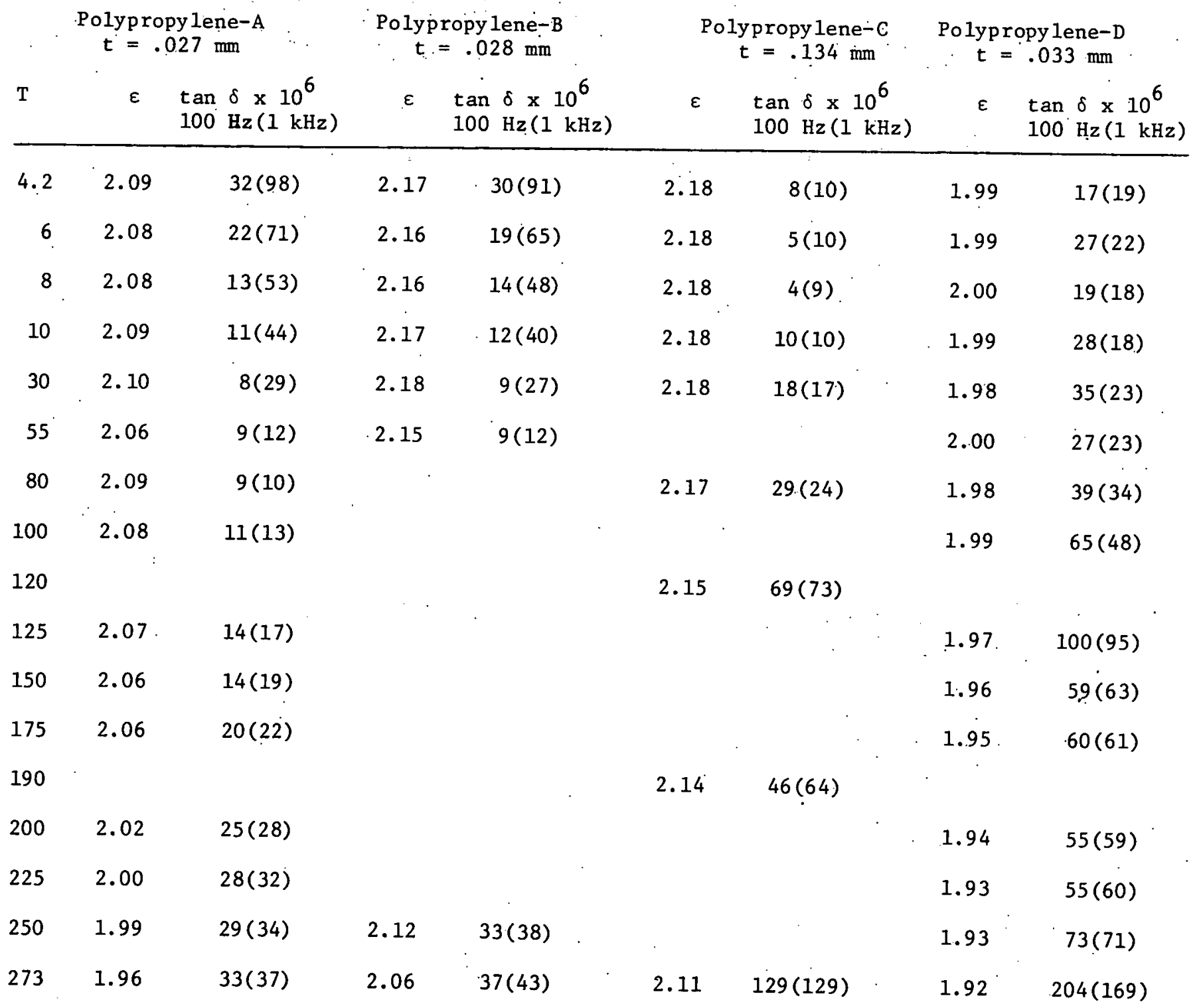


Tens1le Propert1es of Polymer Tapes at $293 \mathrm{~K}\left(\mathrm{BNL}^{33,34}\right.$ )

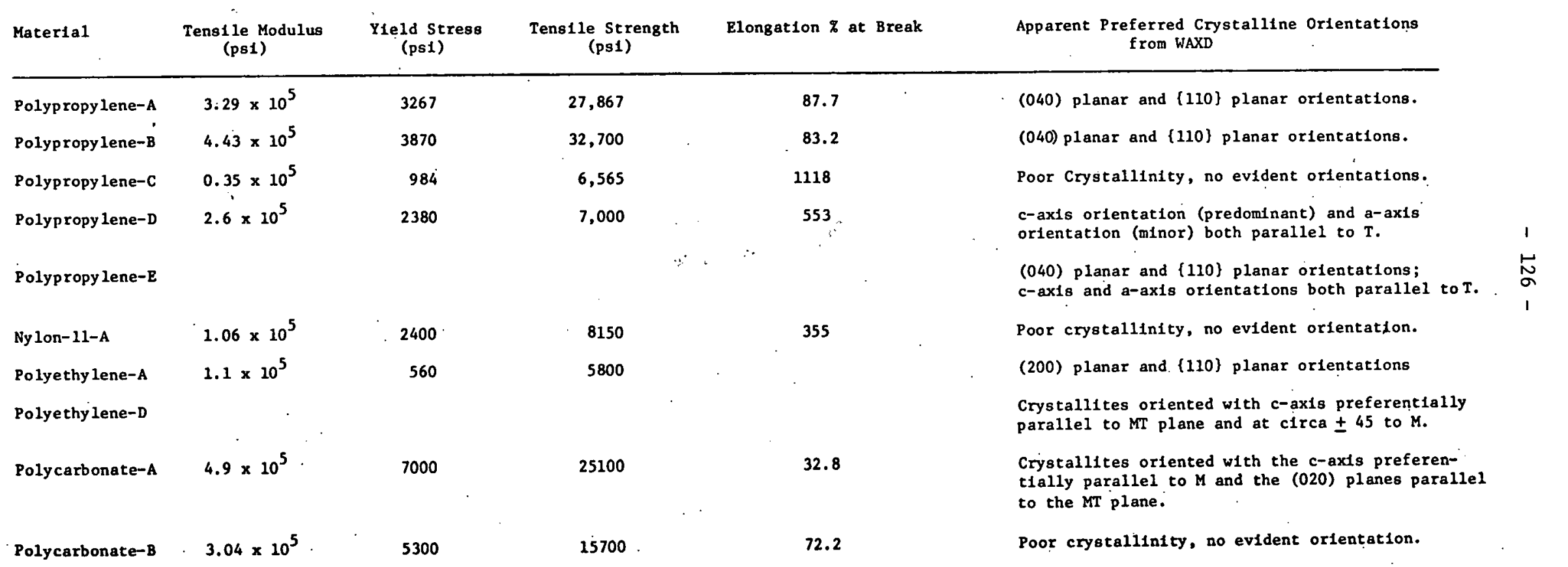




\section{Glossary}

\section{Isotactic Polypropylene:}

Polypropylene is a member of the polyolefin family of polymers, [- $\left.\mathrm{CH}_{2}-\mathrm{CH}-\right]$ where $-\mathrm{R}$ is an alkyl side group. In the case of polypropylene $-\mathrm{R}$ is $-\mathrm{CH}_{3}$. One of three types of stereoisomeric chains of polypropylene (or other polyolefins) can be synthesized depending on the type of catalyst and the conditions used in the polymerization process. The three types of chains differ from one another in the dispositions of the side groups, $-R$, relative to the backbone carbon atoms in the chains. The configurational differences between the three types of chains are demonstrated in Fig. 25 where, for the sake of simplicity in illustration the backbone carbon atoms are depicted to be in a planar zig-zag conformation. Fig. 25a represents an isotactic chain which is characterized by the fact that all the side groups occur on the same side of the plane which contains the backbone carbon atoms. Fig. 25b represents a syndiotactic chain in which there is a regular altemation of successive side groups from one side to the other of the plane containing the backbone carbon atoms. Fig. 25c depicts a chain in which successive side groups occur randomly, on one side or the other, relative to the plane of reference, such chains are referred to as atactic. Isotactic and syndiotactic polypropylene undergo crystallization, atactic polypropylene does not. [A detailed discussion of tacticity in polyolefins and other polymers may be found in "Stereoregular Linear Polymers" by I. Pasquon in "Encyclopedia of Polymer Science and Technology", Vol. 13, pp. 13-86, (Interscience Publishers, New York, 1970)]. 


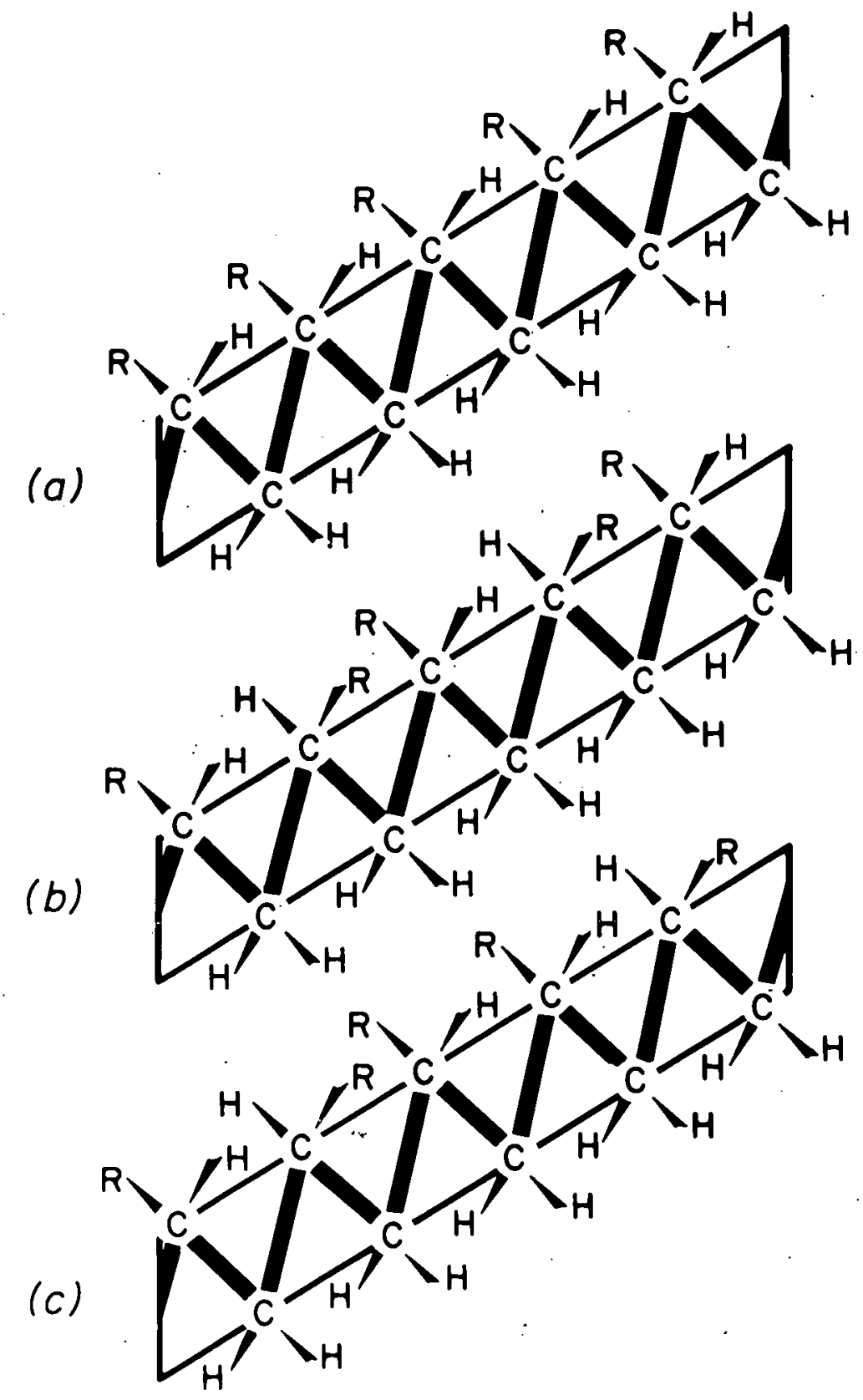

Figure 25; Illustration of the chain configuration in (a) isotactic, (b) syndiotactic and (c) atactic chains in polyolefins (see text). 
Wide Angle and Small Angle X-ray Diffraction (WAXD and SAXD)

In both the wide angle and small angle $x$-ray diffraction experiments described in this report, the polymer samples were exposed to pinhole collimated $x$-ray beams, and the diffracted rays were recorded on photographic film. The terms wide angle and smail angle refer to the range of angles, between the incident $x$-ray beam and the diffracted rays, over which the diffracted rays are recorded. In WAXD the range is from a few degrees to $30-50^{\circ}$, whereas in SAXD the range is from a few seconds of arc up to one degree or so. [A brief introduction to the type of information which can be derived from WAXD and SAXD regarding the fine structure of polymers is given in "Experiments in Polymer Science" by E. A. Collins, J. Bareś, and F. W. Billmeyer, Jr., pp. 191-197. (Wiley-Interscience, New York, 1973). More advanced treatments of the use of WAXD and SAXD in polymer structure characterization are given in "X-ray Diffraction Methods in Polymer Science" by L. E. Alexander (Wiley-Interscience, New York, 1969].

Orthorhombic, Monoclinic, Triclinic:

Crystal lattices are conveniently. grouped according to seven types of conventional unit cells, three of which are the orthorhombic, the monoclinic and the triclinic cells. The unit cell axes are conventionally denoted $a, b$ and $c$, and the angles between these axes are denoted $\alpha, \beta$, and $\gamma$, where $\alpha=b \widehat{c}, \beta=\widehat{a}$, and $\gamma=\widehat{a} \mathrm{~b}$. In orthorhombic cells $\mathrm{a} \neq \mathrm{b} \neq \mathrm{c}, \alpha=\beta=\gamma=90^{\circ}$; in monoclinic cells $\mathrm{a} \neq \mathrm{b} \neq$ $c, \alpha=\gamma=90^{\circ} \neq \beta$; in triclinic cells $a \neq b \neq c, \alpha \neq \beta \neq \gamma$. [For a discussion of the fundamental types of crystal lattices see "Introduction to Solid State Physics", by C. Kittel, pp. 12-33 (John Wiley and Sons, New York, 1971, 4th Ed.)]. 
High Voltage Measurements Section Electricity Division

National Bureau of Standards

Washington, D.C. 20234

Dr. R. K. Eby

Chief, Polymers Division

National Bureau of Standards

Washington, D.C. 20234

Dr. M. Broadhurst

Polymers Division

National Bureau of Standards

Washington, D.C. 20234

Dr. B. Fanconi

Polymers Division

National Bureau of Standards

Washington, D.C. 20234

Dr. F. I. Mopsik

Polymers Division

National Bureau of Standards

Washington, D.C. 20234

Dr. F. Khoury

Polymers Division

National Bureau of Standards

Washington, D.C. 20234

Mr. J. P. Colson

Polymers Division

National Bureau of Standards

Washington, D.C. 20234

Mr. L. H. Boltz

Polymers Division

National Bureau of Standards

Washington, D.C. 20234 
Mr. S. J. Kryder

Polymers Division

National Bureau of Standards

Washington, D.C. 20234

Mr. E. B. Forsyth

Brookhaven National Laboratory

Associated Universities, Inc.

Upton, Long Island, N.Y. 11973

Dr. W. E. Keller

Los Alamos Scientific Laboratory

University of California

Los Alamos., New Mexico 87544

Dr. Mario Rabinowitz

Electric Power Research Institute

3412 Hillview Avenue, P.0. Box 10412

Palo Alto, California 94303

Mr. A. C. Muller

Brookhaven National Laboratory

Associated Universities, Inc.

Upton, Long Island, N.Y. 11973

Mr. A. J. McNerney

Brookhaven National Laboratory

Associated Universities, Inc.

Upton, Long Is.land, N.Y. 11973

Dr. R. W. Meyerhoff

Union Carbide Corporation

Linde Research Laboratories

Tarrytown, N.Y. 10591

Dr. R. J. Densley

Power Engineering Section

National Research Council

Ottawa, Canada KIA OR8 